\title{
HISTOLOGICAL TECHNIQUE
}

\section{UC-NRLF}

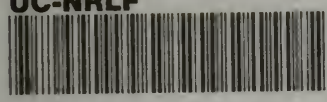

Kares BuRY:

+B $807 \quad 065$ 


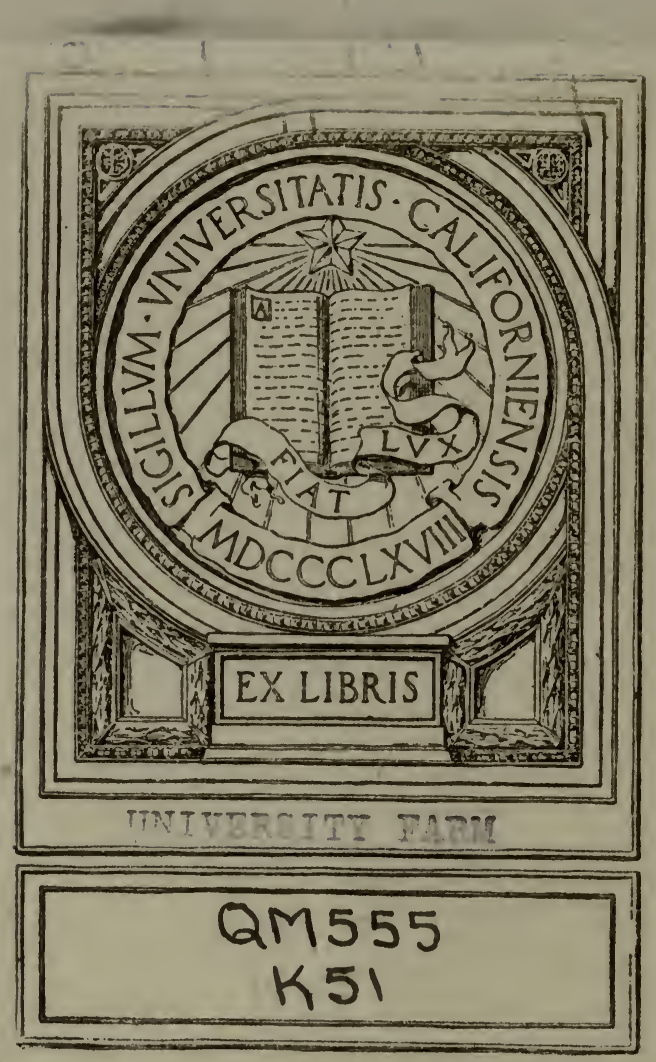


Digitized by the Internet Archive in 2007 with funding from Microsoft Corporation 



\section{HISTOLOGICAL TECHNIQUE}

. BY

B. F. KINGSBURY, Ph.D., M.D.

Professor of Histology and Embryology

1915

CARPENTER \& COMPANY

ITHACA, N.Y. 


\section{NOTE.}

The methods in use in the Department of Histology and Embryology and applied in the instruction, have been in the past twice gathered together and put in printed form for the convenience of students and staff: "Histological Methods," by Professor S. H. Gage; the technique portion of "Vertebrate Histology" by Gage and Kingsbury. In the following presentation the latter of these two has been largely drawn on and certain loose sheets of technical directions by S. H. Gage incorporated. In addition the writer is particularly indebted to The Microscope, by S. H. Gage; The Microtomists Vademecum, by A. B. Lee, and Die Enzyklopaedie der mikroskopischen Technik, together with a large number of original articles, some of which are included among the list of References. When referred to in the text, the reference is indicated by a bracketed number. Acknowledgment is also due to Instructors in the Department, J. A. Badertscher and H. M. Kingery. Their names in parenthesis indicate methods particularly due to them.

B. F. Kingsbury.

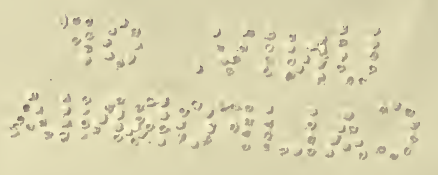

COPYRIGHT

Carpenter \& Co.

1915 


\section{CONTENTS.}

Introduction . . . . . . . . . . . . . . . . . 5

Fixation . . . . . . . . . . . . . . . 7

Fixers, List of . . . . . . . . . . . . . . . . 11

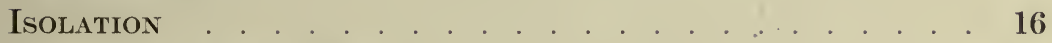

Sectioning \& Imbedding . . . . . . . . . . . . . . . . . . . 19

Schema for Imbedding . . . . . . . . . . . . . . . . . 21

The Paraffin Method . . . . . . . . . . . . . . . . . . 22

The Celloidin Method . . . . . . . . . . . . . . . . . 26

The Freezing Method . . . . . . . . . . . . . . . . . . 33

Staining . . . . . . . . . . . . . . . 34

Stains, List of . . . . . . . . . . . . . . . . . . . 38

Preparation for Staining . . . . . . . . . . . . . . . . 46

Schema for Staining . . . . . . . . . . . . . . 51

Mounting, (Sealing, Labeling). . . . . . . . . . . . . . 52

Slides and Covers, Knife _. . . . . . . . . . . . 57

Special Methods . . . . . . . . . . . . . . . . . . . . 59

The Cell . . . . . . . . . . . . . . . . 59

Connective Tissue . . . . . . . . . . . . . . . 61

Muscle . . . . . . . . . . . . . . . . 66

The Nervous System . . . . . . . . . . . . . 68

The Blood. . . . . . . . . . . . . . . . . . . . . . . 79

Fine Injection . . . . . . . . . . . . . . . . . . . . 82

Silver Nitrate Impregnations . . . . . . . . . . . . . . 83

Histo-chemical Methods . . . . . . . . . . . . . . . 84

References . . . . . . . . . . . . . 90

INDEX . . . . . . . . . . . . . . 93 



\section{INTRODUCTION.}

Very few structures of the animal organism can be adequately examined microscopically without being first subjected to a preparatory treatment involving in many cases the employment of complicated methods. Save in the case of the body fluids and certain membranes, animal tissues are bulky, more or less opaque, and therefore unsuited for examination under the microscope which requires surface or thin layers of substance. Examination is made possible in such cases in one of two ways,-the elements composing the structure may be separated from each other, or thin slices may be prepared.

The above, however, presents but the grosser aspect of the necessity of preparation of animal tissues for examination with the microscope. The histological analysis of bodily structure makes further demands on the refinement of methods. Treatment with chemicals and stains (Fixation and Staining) has for its purpose not only the preservation and delineation of structure, but its identification by means of more or less definite chemical (physical) reactions. The goal from this side of histological technique is an analysis from the chemico-physical as well as the morphological aspect and the interpretation of morphology in terms of physiology. Increase in our knowledge of the finer structure of the body in the past has been, as advance in the future will be, accompanied by and dependent on the application of a more exact technique along these lines; while for those who aim to do practical work in histology and pathology a mastery of the more important methods is indispensable.

Furthermore, in working with chemically altered structure there is always the danger of losing sight of the conditions existent in the living protoplasm. It is well, therefore, in addition to study structure in the living or fresh state, as little altered from the natural as may be. There is also very desirable the acquisition of skill in the application of simple methods which require neither expensive apparatus nor expenditure of time,--methods which while they may not advance knowledge, serve often to meet the needs of a preliminary examination or rapid clinical diagnosis.

Of the multitudinous methods employed in microscopic work only those are here given which meet the requirements for a general working knowledge in histology. In special investigations, it is 
necessary to make a study of the particular technical needs of the problem, and for this it is well to consult the larger works on technique of which may be mentioned the Encyclopedia of Microscopical Technique [6]; The Microtomist's Vade-mecum by A. B. Lee [30]; Physiological Histology, by Gustav Mann [36]; Mallory \& Wright, Pathological Technique [35]: The books of Gage, Hardesty and v. Kahlden-Gierke will also be found valuable for consultation.

General Histological Technique involves then:

A. Examining fresh, by either B. or C. Advantageous or necessary when haste is required, or in examining the tissue alive.

B. Isolation or Dissociation. Separating out the elements composing a tissue by (a) teasing or (b) treatment with reagents and teasing.

C. Cutting thin sections of the tissue or organ.

For C. are generally necessary:

1. Fixing the tissue $(\S \S 1-34)$. Hardening.

2. Sectioning by one of the following methods:

(a) Free-hand, without an imbedding mass, or

(b) With an imbedding mass, as

(1) By the Paraffin method ( $\$ 50)$, or

(2) By the Celloidin method ( $\$ 61)$, or

(3) By the Freezing method ( $\$ 73)$.

D. Staining;-to outline and differentiate the structure, or pick out definite chemical substances.

E. Mounting;-for examination under the microscope and permanent preservation.

In addition,--numerous

F. Special Methods and methods for the

G. Histo-chemical Analysis of structure must be frequently applied. 


\section{FIXATION.}

$\S 1$. Fixation is one of the fundamental processes in the examination of plant and animal tissues. A fixer may be defined as a fluid (or gas) into which the living or at least very fresh tissue is placed in order to preserve the structure of its elements as nearly as possible as in life. Living tissue when allowed to die and remain undisturbed, gradually loses the structural features it had in life and undergoes disintegration and decay. Fixation depends upon physico-chemical processes wherein the chemical constituents of the tissue are thrown down in situ by being rendered insoluble in some form or represented by substitution products; the whole being attended by as little distortion as possible. It should be appreciated that the chemical constituents of cell protoplasm and of the tissues are numerous and diverse in their chemical and physical properties, so that a universal or ideal fixer not only does not exist but is logically inconceivable. The bulk of protoplasm and the tissues is protein and the basis of fixation in general is the precipitation or coagulation of these chemical substances. It should be remembered however that fats (lipoids*) are a constant though variable component of cytoplasm; that carbohydrates (glucose, glycogen, etc.) are usually present in small amounts, and that the products of cell activity such as secretion-granules, zymogen, etc., may be quite distinctive in their physico-chemical properties. Within the cell a certain "antagonism" exists between nucleus and cytoplasm, the former oxidative, the latter reducing, requiring often somewhat different fixation conditions.

For the best results, the fixer should be chosen with a view to the preservation of some particular part or constituent, though a number of general fixers are very serviceable for routine work. Rational fixation will depend upon a detailed knowledge of the chemical and physical properties of the constituents that it is desired to preserve and their interaction with the chemicals of the fixer. In many respects, rational fixation still awaits further knowledge of the physics and chemistry of fixation.

$\S 2$. The chemicals of most service as fixers are: (1) osmic acid (osmium tetroxid), (2) platinic chlorid, (3) picric acid (tri-

*Lipoid, while not a good chemical term is one that is quite useful in histology, to include fats, fatty acids, phosphatids, cholesterol, etc., substances that have the same solvents and which are found associated in protoplasm. 


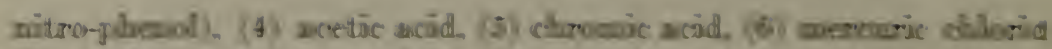

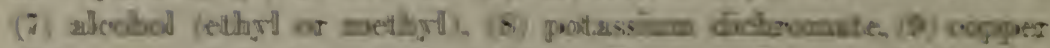

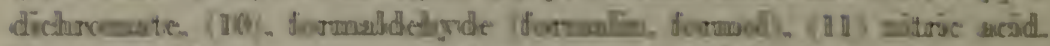

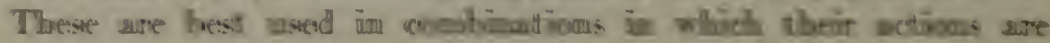

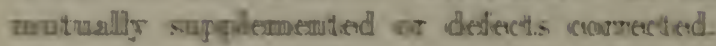

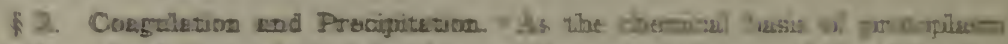

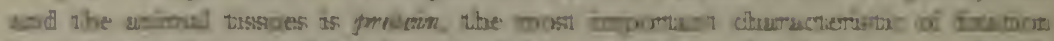

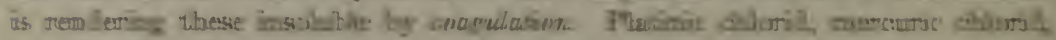

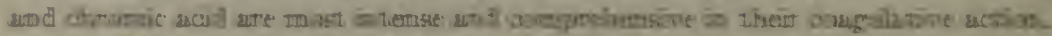

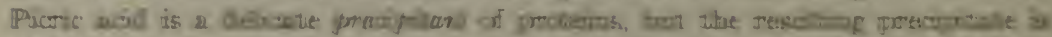

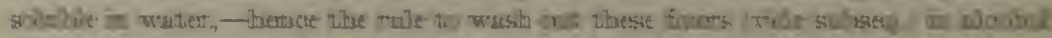

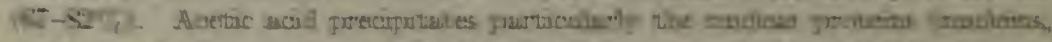

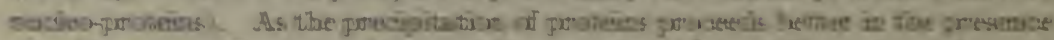

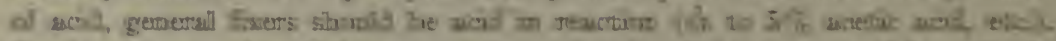

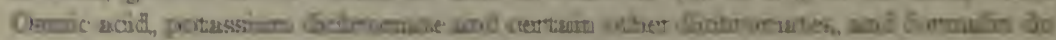

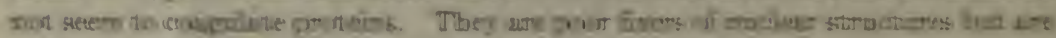

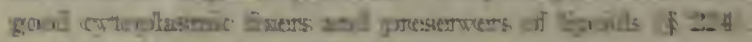

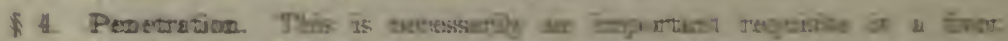

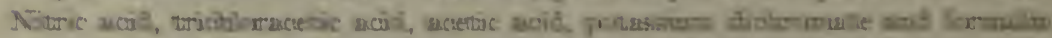

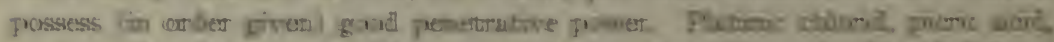

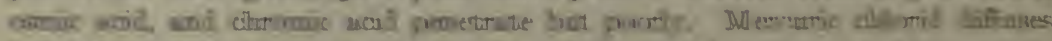

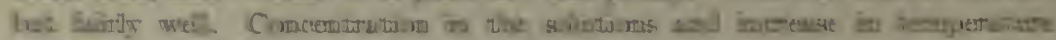

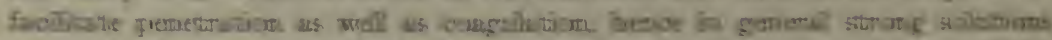

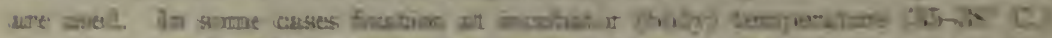

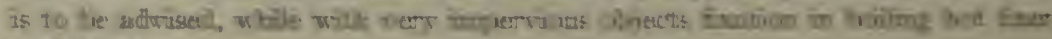
Is wolrisal ile

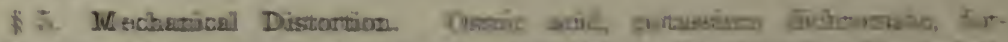

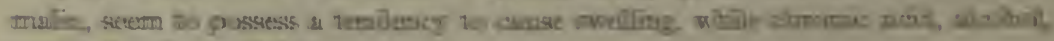
minstite the tassue sthrist:

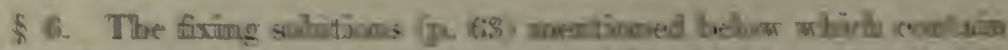

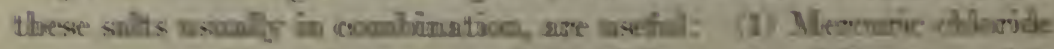

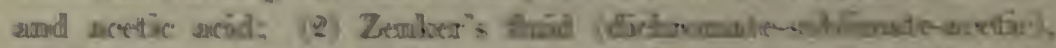

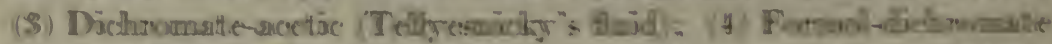

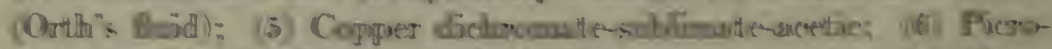

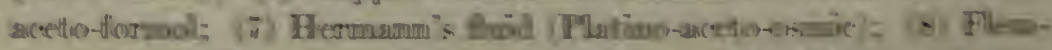

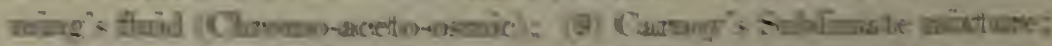

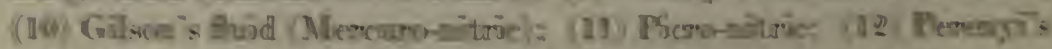

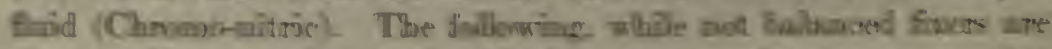

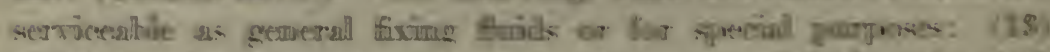

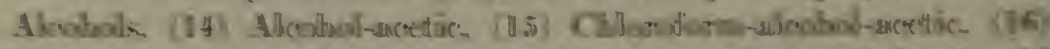

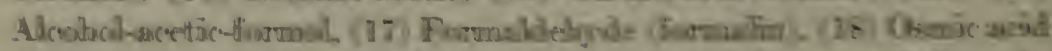

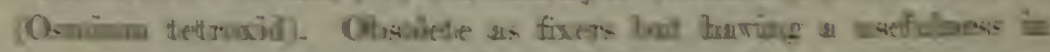

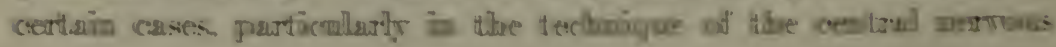


system ( $§ 199,201,204)$ are; (19) Müller’s fluid, (20) Erlicki’s fluid (21) Potassium dichromate (in aqueous solution).

In the combination of chemicals in fixing solutions such as those mentioned above, they should be chosen to supplement each other's actions as far as possible and correct or counteract their defects. The combinations must be chosen always with a view to the result desired and frequently the components and their relative amounts determined empirically,-by experiment.

$\S 7$. The following general rules should be regarded in the fixation of tissues and organs:

(1). The volume of the fixing fluid used should be large, exceeding the volume of the tissue at least thirty times. The less energetic the action of the fixer the greater the amount of fluid to be employed. When the fluid becomes turbid it should be changed to fresh at once.

(2). Fix only as small pieces of tissue as possible, or as is practicable from the results desired. The block of tissue should not be more than $1 \mathrm{~cm}$. in one dimension, and if possible let it be much shorter, - only 2 to $5 \mathrm{~mm}$. In some cases (Flemming's and Hermann's fluids) much smaller masses are needed ( 1 to $2 \mathrm{~mm}$. thick). This is desirable for the rapid and complete penetration of the fixer. Of course, in the case of entire organs it may not be possible to comply with the conditions. Fixation by injection may then be resorted to. Physiological salt solution ( $\$ 35$ ) is first injected through artery of the organ or part in order to remove the blood, and this is followed by the injection of the fixing fluid.

In addition to these two general principles, there are four points to be carefully considered, upon which the excellence in the results attained depends; they are (a) the fixer chosen, (b) the time of fixation, (c) the washing out of the fixer, (d) the hardening in alcohol and the subsequent treatment.

(a) The choice of a fluid into which the tissue is placed should be made dependent on (1) a consideration of the particular feature whose preservation is desired and the degree of excellence of fixation that is desired or necessary,-whether detail of cell structure or the structure of the tissue in terms of cells or structural elements be sought; (2) the penetrating power and the size of the piece that it is 
necessary to have; and (3) the stain that is desired subsequently which is largely determined by the fixation.*

(b) The time a fixer is allowed to act should be considered in connection with the character of the fluid and the tissue. Usually the exact limitation of time is a matter of secondary importance and the tissue may remain in the fixer indefinitely. In some cases, however, its disregard affects the results seriously and as a general rule there is a minimum and a maximum time and between them an optimum time that should be adhered to.

(c) After the tissue has been in the fixing fluid a proper length of time, it is necessary that it be washed thoroughly to remove the fixer from it. Usually this should be done by means of water or alcohol or both. In general, fixers containing salts insoluble in alcohol or but slightly soluble, as osmic acid, chromic acid, potassium dichromate, etc., should be thoroughly washed in water. Fixers containing picric acid or alcohol should always be removed by alcohol; mercuric chlorid may be washed out by either water or alcohol.

Inadequate washing out of the fixer may either seriously affect the cutting quality of the tissue (if it is to be subsequently imbedded), the ease with which it can be stained, or there may be formed precipitates in the tissue giving illusory effects, distortions, or at least a dirty appearance to the preparation. Time in properly washing out a fixer is always well spent, as it is a matter for serious attention.

§ 8. Resume. In brief, then: In fixing, take relatively large amounts of fluid and small pieces of tissue, choose the fixer well with a view to the tissue and the results desired, permit the fixing to proceed for a sufficient length of time, and wash out thoroughly.

HARDENING AND STORING.

§ 9. Each fixer has also more or less of a hardening action upon the tissue. Some fluids spoken of above as fixers were primarily used as hardeners, such as Müller's fluid or Erlicki's fluid, while with others, e. g., picric acid in aqueous solution, the hardening action is a minimum. The hardening action of the fixer

*Numerous papers have been written on the nature of fixation and the action and relative value of the various chemicals used for that purpose. The contributions of Berg [4], Fischer [7], Mann [36], and v. Tellyesniczky [6] may be particularly mentioned. 
is generally supplemented by the subsequent use of alcohols of increasing strengths ( $50 \%$ to absolute, $-99 \%$ ), as well as in preparation for the paraffin and celloidin methods of imbedding. In fact, with modern methods of imbedding excessive hardening of the tissue is not necessary and indeed often should be avoided as affecting the cutting quality of the tissue. Tissue after fixation has been completed may be stored in 82 or $95 \%$ alcohol, or (better) imbedded at once $(\$ 47)$.

Alcohols. $50 \%, 67 \%$, and $82 \%$ alcohols form a series of increasing strengths sufficient for most purposes. They may be prepared from $95 \%$ alcohol by taking -(a) for $50 \%$ alcohol; $95 \%$ alcohol 1 part, water 1 part; (b) for $67 \%$ alcohol; $95 \%$ alcohol 2 parts, water 1 part; (c) for $82 \%$ alcohol, $95 \%$ alcohol 5 parts, water 1 part. Dilutions of other strengths may easily be prepared as desired from $95 \%$ alcohol. $95 \%(94 \%)$ alcohol and absolute alcohol are necessary in imbedding by the paraffin and celloidin methods ( $\$ 49-$ ).

$\S 10$. Stock Solutions. It is advantageous to have on hand strong solutions of the chemicals employed as fixers and stains. Where feasible, $10 \%$ solutions are most convenient. The following are the more important: In aqueous solution;-10\% potassium dichromate, 10\% copper dichromate, $10 \%$ chromic acid, $10 \%$ platinic chlorid, $40 \%$ formaldehyde (formalin), $4 \%$ sodium sulphate, $4 \%$ copper sulphate, $2 \%$ osmic acid, saturated solution of mercuric chlorid, saturated solution of picric acid, $95 \%$ alcohol, absolute alcohol, etc., as well as the strong acids, stock staining solutions, etc.

\section{Fixers}

§ 11. Mercuric chlorid. One may employ (a) a saturated solution in water or $(b)$ a saturated solution in normal salt solution, with 1 to $5 \%$ glacial acetic acid. Water will dissolve about $5 \%$, normal salt solution about $12 \%$ of the mercuric chlorid. This is a good fixer, especially when the piece is small. It fixes as soon as it penetrates and is apt to make tissue brittle if it is left too long. Staining after it is brilliant. The larger percentage of acetic acid is, perhaps, to be preferred for most histological objects.

Fix the fresh tissue $1 / 2$ to 24 hours according to the size of the piece. Remove to $67 \%$ alcohol for 1 or 2 days, $82 \%$ alcohol several days, changing often. The $82 \%$ alcohol should contain enough tincture of iodin to give it a yellow color, and fresh tincture added or the alcohol changed when the yellow color of the iodin in the alcohol is lost. As long as the alcohol is decolorized, washing should be continued, since it is important that the mercuric chlorid be all removed from the tissue; otherwise precipitates will form in the preparation after it is mounted or before, and spoil the result. Wash out in alcohol thoroughly and carefully. Almost any stain may be employed after a mercuric chlorid fixation.

$\S 12$. Zenker's fluid. Formula: Potassium dichromate, 2.5 grms.; sodium sulphate, 1 gram; mercuric chlorid, 5 grms.; water, 100 c.c.; and add before using, glacial acetic acid, 5 c. c. A stock solution, without the acetic acid should be kept on hand. This is a well balanced fixer; the potassium dichromate seems to check the brittleness that the mercuric chlorid would cause and improves the fixation of the cytoplasm while the mercuric chlorid and acetic afford a good nuclear fixation. It is distinctly better than mercuric chlorid; staining after it, however, is apt to be a little more difficult and not as brilliant as with mercuric 
chlorid fixation. Its penetration is surprisingly good. The sodium sulphate is probably unnecessary. Use of physiological $(0.6 \%)$ salt solution instead of water to prevent the formation of precipitates or the addition of a small amount of salt to the fluid when made up for use, is recommended by some.

Fix in Zenker's fluid 12 to 48 hours, wash well in water, running or frequently changed, 12 to 48 hours, to remove the dichromate; transfer to $67 \%$ alcohol for 1 or 2 days, $82 \%$ alcohol for several days, keeping in the dark while in the alcohol. To the $82 \%$ alcohol add a drop or so of tincture of iodin, adding fresh iodin or changing the alcohol when the color is lost. This should be continued as long as the iodinized alcohol is decolorized in order to avoid a precipitate of the mercuric chlorid in the tissue. Avoid, however, adding an excess of iodin, since it will affect the staining quality of the tissue. Stain as you wish.

$\S 13$. Helly's fluid. (Zenker-formol). Formula: Zenker's fluid, with formalin substituted for the acetic acid. Potassium dichromate, 2.5 grams; sodium sulphate, 1 gram; mercuric chlorid, 5 grams; formalin, 5 or 10 c. c., to be added before using. Valuable for the fixation of cytoplasm and cytoplasmic granules, where the acetic acid is to be avoided. Make up from the Zenker's stock by adding the formalin.

Fix 6 to 24 hours or longer, wash in running water 6 to 12 hours, alcohols. If it is desired to give a longer mordantage in dichromate, transfer to Müller's fluid or other simple dichromate solution.

$\S 14$. Dichromate-acetic. (Tellyesnicky's fluid) Potassium dichromate, 3 grms.; glacial acetic, 5 c. c.; water, 100 c. c. This fluid gives good preservation of nuclei and of the cytoplasm, the acetic acid checking the bad effects of the dichromate. A simpler fixer than Zenker's fluid.

Fix 1 to 14 days according to the size of piece and the object. Wash in running water 12 or 24 hours, and pass up through $25,50,67$, and $82 \%$ alcohols, 12 to 24 hours in each.

$\S 15$. Formol-Dichromate (Orth's fluid). Formula: Potassium dichromate, 2.5 grms.; sodium sulphate, 1 grm.; water, 100 c. c. (i. e., Müller's fluid); formalin, 10 c. c. A good fixer of cytoplasm, but an indifferent one for nuclear detail. It has been especially used in the case of the nervous system ( $\$ 199$ ). Other proportions of formalin and dichromate may be taken, the sodium sulphate being omitted. It should be freshly made up as it soon deteriorates.

Fix 3 to 24 hours or longer, wash in running water 12 to 24 hours; 50,67 , $82 \%$ alcohols, 1 day in each. If a long fixation is given, the fluid should be frequently changed.

$\S 16$. Copper dichromate-sublimate-acetic. Formula: $10 \%$ copper dichromate, 1 part; $4 \%$ copper sulphate, 1 part; sat. solution mercuric chlorid, 2 parts; glacial acetic acid, $\frac{1}{10}$ to $5 \%$, according to need. Similar to Zenker's fluid in its general properties and should be used in the same way. Excellent as a preserver of cytoplasmic granules of lipoid nature $(\$ 177-9)$. A further mordantage in $2.5 \%$ copper dichromate is often advantageous (3 to 4 days).

By the substitution of formalin (5 to $10 \%$ ) for the acetic acid, a fixer comparable to Helly's fluid $(\$ 13)$ is obtained.

$\S 17$. Picro-aceto-formol. (Picro-formol). Formula: Picric acid, saturated aqueous solution, 75 c. c.; formalin, 25 c. c.; glacial acetic acid, 4 c. c. 
A delicate fixer useful in some cell work and with small objects. The formalin may be omitted (Picro-acetic).

Fix 3 to 24 hours, transfer to $67 \%$ alcohol, 1 day, $82 \%$ alcohol several days changing the fluid frequently as it becomes yellow. Leave in the alcohol until the picric acid has been well washed out.

§ 18. Hermann's fluid. Formula: $1 \%$ aq. sol. platinic chlorid, 15 parts; $2 \%$ aq. sol. osmic acid, 4 parts; glacial acetic acid, 1 part; or you may take $10 \%$ aq. sol. platinic chlorid, 3 parts; $1 \%$ aq. sol. osmic acid, 16 parts; glacial acetic acid, 2 parts; water, 19 parts. This is generally recognized as one of the finest fixers known, and it is also the most expensive. The form and structure of cells are well preserved. It should only be employed, however, with very small pieces of tissue, and is to be used especially when cell structure is to be studied. Fat and the myelin of nerve fibers are stained black.

Fix in this 1 to 24 hours (or longer-days or weeks are used by some), wash well in water (running or frequently changed) 2 to 24 hours, and then place in $67 \%$ and $82 \%$ alcohols, 12 to 24 hours in each. In using this fluid, the smaller the pieces taken the better the fixation will be, and in order that it be possible to obtain a good stain afterwards tissue should not be over-fixed and the fixer should be thoroughly washed out. If there is a blackening of the tissue, or a precipitate in it, both may be removed by treatment of the sections on the slide with a 10 or $20 \%$ solution of hydrogen dioxid in $67 \%$ alcohol, or with perhydrol (Merck). Employ after Hermann's fluid, as stains, Heidenhain's iron hematoxylin, Delafield's hematoxylin, safranin (as a red stain), or gentian violet (as a blue stain), or neutral stains.

$\S 19$. Flemming's fluid (Chrome-aceto-osmic). Formula: $1 \%$ aq. sol. chromic acid, 15 parts; $2 \%$ aq. sol. osmic acid, 4 parts; glacial acetic acid, 1 part; or, $10 \%$ aq. sol. chromic acid, 3 parts; $1 \%$ osmic acid, 16 parts; glacial acetic acid 2 parts; water, 19 parts. This is a fine fixer and in most cases gives as good results as Hermann's, and is not as expensive. It browns tissue less, and while it blackens fat, does not blacken the myelin of myelinic nerve fibers as does Hermann's. It should be employed in general in the same cases and in the same way as Hermann's fluid, and is especially useful in the preservation of free fats and lipoids $(\$ 227)$. The presence of chromic acid gives it a distinct value as a cytoplasmic fixer.

Fix tissue 1 to 24 hours (or longer); wash well in running water 2 to 24 hours; place in $67 \%$ and $82 \%$ alcohols, 12 to 24 hours in each. Bleaching of the sections may be necessary, as with Hermann's fluid. Take only very small pieces of tissue. Employ the same stains as with Hermann's fluid.

The reduction of the acetic acid to 3 or 4 drops (about $\frac{1}{10} \%$ ) is advisable when cytoplasmic granules of lipoid composition $(\$ 178)$ are to be preserved. The fluid may then be spoken of as Benda's fluid.

§20. Mercuro-nitric (Gilson's fluid, modified). Formula: Nitric acid $\left(46^{\circ}\right.$, sp. gr. $1.456,80 \%$ ), 15 c. c.; Glacial acetic acid, 4 c. c.; Mercuric chlorid, 20 grms.; $95 \%$ alcohol, 60 c. c.; distilled water, 920 c. c. A good fixer, especially useful where rapid penetration is a factor, and as a fixer of cells rich in yolk (amphibian ova, etc.).

Fix 12 to 24 hours, transfer to 50,67 , and $82 \%$ alcohols. Iodin should be used to ensure the removal of all the mercuric chlorid (see $\S 11$ ). 
\$21. Picro-nitric. Formula: Distilled water, 95 c. c.; nitric acid (strong), 5 c. c.; picric acid, to saturation. Only to be recommended for eggs rich in yolk and where a high power of penetration is required.

Fix 4 to 12 hours, transfer to $67 \%$ alcohol, 1 day $82 \%$ alcohol several days changed frequently. It is necessary to wash out thoroughly which is accomplished however, with difficulty.

\$22. Perenyi's fluid. Formula: $10 \%$ aq. sol. nitric acid, 4 parts; $95 \%$ alcohol, 3 parts; $1 / 2 \%$ aq. sol. chromic acid, 3 parts. An embryological fixer of much value. It is also serviceable for general work.

Fix tissue for 4 to 5 hours, place in $67 \%$ alcohol for 1 day, $82 \%$ alcohol several days.

§ 23. Alcohol. $95 \%, 67$ or $70 \%$ alcohol.

The employment of most of the fixers so far mentioned requires the expenditure of considerable time, rendering them inapplicable or unsuitable in many instances. $95 \%$ alcohol itself answers admirably for most histologic objects, fixing well, hardening and likewise dehydrating ( $\$ 49)$ preparatory to imbedding in paraffin or collodion, affording thus a considerable economy of time. It is also most serviceable in pathological tissues where the presence of bacteria is suspected. In some instances $67 \%$ alcohol answers as well or better, while in other cases absolute $(99 \%)$ alcohol should be employed.

Fix in $95 \%$ alcohol for 1 to 3 days, changing two or three times, after 3 or 4 hours and after 24 hours. The tissue will probably be found to be dehydrated and ready for the next step of the imbedding process ( $\$ 51$ or 62 ). Stain as you wish.

§ 24. Alcohol-acetic. The addition of $5 \%$ glacial acetic acid to $95 \%$ alcohol or absolute alcohol increases the penetrating power and improves the cutting quality of objects containing much connective tissue. The following formula possesses high penetration and is sometimes useful:

$\S 25$. Alcohol-acetic (Carnoy's fluid, 3-1). Formula: Glacial acetic acid 1 part, $95 \%$ or absolute alcohol, 3 parts. Transfer after fixing to $95 \%$ or absolute alcohol, changing each day until the acetic acid is well washed out.

$\S 26$. Chloroform-alcohol-acetic. (Carnoy's 6-3-I ). Formula: $95 \%$ or absolute alcohol, 6 parts; chloroform, 3 parts; glacial acetic acid, 1 part. Fix 2 to 24 hours or longer, transfer to $95 \%$ alcohol, changed two or three times.

$\S 27$. Carnoy's Sublimate mixture. The above $(\$ 26)$ with mercuric chlorid added to saturation. A very penetrating and excellent fixer, particularly useful for nuclear preservation and for embryos.

Fix 1 to 24 hours, transfer to $95 \%$ alcohol for 1 day, then to $82 \%$ alcohol for several days, changing daily and adding iodine solution ( $\$ 11)$ until the excess mercuric chlorid is removed.

$\S 28$. Alcohol-acetic-formol. Combinations such as the following are useful for general purposes. V. Luko's formula: Alcohol, $67 \%$, or $82 \%, 100$ c. c. glacial acetic acid, 5 c. c.; formalin, 5-10 c. c.

Fix for 12 to 24 hours or longer; remove to $67 \%$ or $82 \%$ alcohol for 1 day or more.

\$ 29. Formaldehyde. Solutions of this chemical have been found to be good preservatives and hardeners and fairly good fixers. It penetrates rapidly, 
and preserves the natural transparency and pigmentation of the tissue, making it valuable for gross anatomy and museum purposes. As a fixer, an aqueous solution of 2 to $4 \%$ strength may be employed, or it may be used, which is preferable, in conjunction with other chemicals, as picric acid, in picro-formalin, or chromic acid and acetic acid.

Formalin is a 36 to $40 \%$ solution of formaldehyde (gas) in water. A small amount of formic acid is also present. A $10 \%$ solution of formalin, that is a $4 \%$ solution of the formaldehyde is a satisfactory strength for most histological purposes.

Fix 12 to 24 hours, remove to $67 \%$ alcohol for a day, $82 \%$ alcohol one to several days. Stain as you wish.

$\S 30$. Osmic acid. A very useful as well as expensive reagent and somewhat difficult to use. It is generally employed as a fixer in conjunction with other reagents, as in the mixtures $(\S \S 18$ and 19$)$. When used alone as a fixer weak solutions are generally best- $\frac{1}{10}$ to $1 \%$. It penetrates slowly and it "over-fixes" cells very easily, obscuring detail and giving the parts a homogeneous, glassy appearance. Over-fixed cells cannot be stained, or with great difficulty. More or less blackening of the protoplasm also occurs. It may be used chiefly to demonstrate fat, which is blackened by it, and the zymogen of pepsin and trypsin, which it preserves and browns slightly.

Fix small (about $2 \mathrm{~mm}$. thick or less) pieces of tissue in $1 \%$ osmic acid for 6 to 12 hours, wash well in water (running or changed frequently) for 12 to 24 hours, and place in $67 \%$ and $82 \%$ alcohols. It is somewhat difficult to prevent pure osmic acid of this strength from over-fixing the tissue, and cell detail is generally lost, though the form of cells is well preserved.

$\S 31$. Nitric acid. A $10 \%$ solution of nitric acid is serviceable in fixing the blastoderm of the chick.

$\S 32$. Müller's fluid. Formula: Potassium dichromate, 2.5 grams; sodium sulphate, 1 gram; water, 100 c. c. Make up from stock solutions. This fluid is more of a hardener than a fixer; it should be avoided (as likewise Erlicki's fluid and potassium dichromate) when the preservation of nuclear structure is desired. Staining after its use is sometimes difficult. It is, however, occasionally useful for general work, although such formulas as Zenker's fluid or Helly's fluid are generally to be preferred.

Place the object in an abundance of the fluid and harden for from 1 to 8 weeks, changing the fluid at first each day. In general, 10 to 14 days will be sufficient. Wash in running water for 24 to 48 hours or longer, remove to $67 \%$ alcohol for 1 to 2 days, $82 \%$ alcohol several days. Keep in the dark while in the alcohols, and change to fresh when the fluid is colored yellow. Tissue hardened in Muller's fluid cuts well, and it is useful in preparing sections of large organs, or organs with much connective tissue. Its chief usefulness is, however, in the study of the nervous system ( $\$ 199-)$.

$\S 33$. Erlicki's fluid. Formula: Potassium dichromate, 2.5 grams; copper sulphate, 1 gram; water, 100 c. c. Make up from stock solutions. This is quite similar to Müller's fluid in its action and results, save that its action is more rapid and stronger. Therefore, it had better be employed with smaller objects, and allowed to act only 2 to 14 days. Otherwise, employ like Müller's fluid. 
$\S 34$. Potassium dichromate. $2 \%, 3 \%$, and $5 \%$ aqueous solutions. This is quite similar to Müller's fluid in its action, and may be employed in the same cases. It is generally used for the central nervous system.

Harden in an abundance of the solution for 2 to 8 weeks, beginning with the $2 \%$ solution for 2 to 6 days, $3 \%$ solution 1 to 3 weeks, $5 \%$ solution 1 to 3 weeks. Wash out in running water 24 to 48 hours. Place in $67 \%$ and $82 \%$ alcohols several days, keeping in the dark meanwhile, changing when the alcohol is colored.

\section{ISOLATION.}

$\S 35$. One of the simplest ways of examining the structure of a tissue is the separation from one another of the structural elements composing it, thus permitting its analysis. Likewise, for a correct conception of the forms of the cells and fibers of the various tissues of the body, one must see these elements isolated and thus be able to inspect them from all sides. It frequently occurs also that isolation is not quite complete and one can see in the clearest manner the relations of the cells or fibers to one another.

In the employment of this method the tissue may be taken fresh and isolation accomplished by teasing with needles or similar instruments; or it may be treated with media which will serve to render teasing partially or entirely unnecessary. In such cases simply shaking or gently tapping the preparation will often suffice. In many instances it is desired to examine the tissue while the elements are still alive, as, for example, in the study of ciliated cells, and recourse must be had to some "normal," "indifferent" medium. Best of all is the medium with which they are bathed during life-in the case of tissue from the animal body, blood serum, the aqueous humor of the eye, liquor amnioticus, or, as an artificial substitute often more convenient if reagents are to be used subsequently, physiological or normal salt solution, being a $6 / 10$ to $9 / 10 \%$ solution of common salt (sodium chlorid) in distilled water. $6 / 10 \%$ is suitable for use with Amphibian tissue; $75 / 100 \%$ is normal for reptiles and $9 / 10 \%$ best for mammals. Other normal physiological solutions may in some cases be preferable, such as Ringer's solution, Locke's solution, etc.

$\S 36$. The use of chemical solutions to facilitate isolation of the elements is called Maceration. The chemical agents or solutions for isolating are, in general, the same as those used for fixing and hardening. But the solutions are only about one-tenth as strong as for 
fixing and the action is very much weaker and requires from one or two hours to as many days. In the weak solution the cell cement or connective tissue is softened so that cells and fibers may be separated from one another, and at the same time the cells are preserved. In other words, a weak fixing action is retained while the hardening action is reduced on dilution. The time required for the action of the dissociator varies inversely as the vehemence of the fixer and the density of the tissue, 2 to 3 hours to several days. In fixing and hardening, on the other hand, the cell cement, like the other parts of the tissue, is made firmer. It is better also to dilute the fixing agents with normal salt solution than merely with water [15]. Those chemicals that are "cytoplasmic fixers" such as potassium dichromate, Müller's fluid, formaldehyde, osmic acid, appear to be especially useful in the dissociation of epithelia.

For the isolation of muscle, alkalis or mineral acids, which soften or dissolve the connective tissue are to be employed. Horn, nail and hair require strong mineral acid or (usually) weak alkali.

$\S 37$. Of the many maceration fluids or dissociators the following may serve most of the needs of histology: (1) Müller's fluid dissociator; (2) formaldehyde dissociator; (3) osmic acid (1/10\%); (4) sulphuric acid (strong); (5) nitric acid dissociator; (6) caustic potash dissociator.

§ 38. Müller's fluid dissociator. Formula: Müller's fluid, 1 part; physiological salt solution, 9 parts (i.e., potassium dichromate, 2.5 grams; sodium sulphate, 1 gram; sodium chlorid, 9 grams; water, 1,000 c. c.), [15].

This is a good dissociator for épithelia, including glands. Dilution decreases the hardening action of Müller's fluid as is shown especially on the cell-cement,- -hence its dissociating action. Considerable latitude in time is allowed in the use of this dissociator; 12 hours being often sufficient, although a stay of several days in the dissociator usually does no harm.

§ 39. Directions for use. In the employment of this fluid for the isolation of epithelial cells, proceed as follows:

Place the tissue covered with the epithelium which it is desired to isolate in the dissociator in a shell vial or dish, where it may remain from 2 to 3 hours to 2 to 3 days; for the epithelium of the trachea, intestines, etc., the action is sufficient in 2 to 3 hours, although good preparations may be obtained after two days or more. For the 
stratified epithelia, like those of the skin, mouth, etc., it may require 1 to 3 days for the most satisfactory preparations. After the tissue has remained in the dissociator a sufficient time, scrape the epithelial surface gently with a scalpel and place the scrapings on a slide in a drop of dissociator; cover and examine. If one proceeds after two hours or so, probably most of the cells will cling together, and in the various clumps will appear cells on end showing the tops or bases, and other clumps will show the cells in profile.

Tap the cover gently with a needle-holder or other light object in order to separate the cells from each other more completely. Many fully isolated cells as well as cells in groups will be seen. Examine carefully.

$\$ 40$. Staining. Scrape gently the epithelial surface in a fresh spot and place the scrapings on the slide in a drop of eosin ( $\$ 114)$ or congo red ( $\$ 113)$. Mix well so that the stain can penetrate. If for temporary examination, cover immediately and examine as before. For methods of making permanent preparations of dissociated cells and mounting in glycerin, see $\$ \S 151-$.

$\S 41$. Formaldehyde Dissociator. Formula: $40 \%$ formaldehyde (formalin), 2 c. c.; physiological salt solution, 1,000 c. c. (i. e., $.08 \%$ sol. of formaldehyde in normal salt solution). This is a good general dissociator and as such may be employed instead of Müller's fluid dissociator. It is especially serviceable in the isolation of the nerve cells of the brain and spinal cord [13], and for its use with that material, see $\$ \S 151-$. It is however excellent for the dissociation of epithelia.

$\S 42$. Osmic acid. A $1-10 \%$ solution of osmic acid is a valuable dissociator, especially serviceable in the isolation of nerve-fibers, myelinic and amyelinic, and when fat is present, since fat and the myelin of myelinic nerve-fibers are blackened by it. Twelve to twenty-four hours generally affords sufficient time for it to act. ( $(193$, 227).

$\S$ 43. Sulphuric Acid. This is used in the concentrated form as a dissociator of the epithelial cells of hair, horn, and nail. If heated, a few minutes suffice; employed cold, a day or two may be required.

\$ 44. Nitric acid dissociator. [8] Formula: Strong nitric acid, 20 c. c.; water, 80 c. c. See $\$ 189 a$. This fluid is employed in the isolation of muscle fibers, both striated and plain. 
§ 45. Caustic potash dissociator. [8] Formula: Caustic potash, potassium, hydroxid (in sticks), 35 to 40 grams; distilled water, 65 or 60 c. c. This solution will be used for the isolation of cardiac muscle 'cells', although it may be used for striated or plain muscular tissue, or as a general dissociator. It may also be employed for isolating the cells of hair, horn or nail, either full strength or diluted.

Ten to fifteen minutes or longer will be enough for the isolation of heart muscle ( $\$ 189 b)$; 2 to 3 days may be required for the adequate maceration of cornified epithelial cells.

\section{SECTIONING.}

$\S 46$. In addition to the examination of tissue by the separation of the component elements-isolation-it may be examined microscopically after cutting very thin slices or sections of it. This may be done free-hand or by means of a special machine, a microtome, and with or without an imbedding and supporting mass.

For the finer work an imbedding mass and a microtome must be used. Free-hand sectioning without an imbedding mass, and even without previous fixing is, however, necessary or advisable when economy of time is a desideratum, as in clinical examinations of tissue, when one wishes to study the part alive or fresh (i. e., not treated with reagents), or if the reagents necessary for fixing and imbedding destroy or alter the structural features to be investigated.

The ability to recognize tissues and organs unaffected by reagents and without the employment of methods involving the expenditure of time and effort is very desirable, especially in pathological work, when haste often forbids the employment of the finer methods, were facilities for their use available, as in some cases they are not. Great skill in the use of simple tools may be gained and counts for much. It should be remembered also that the greater one's knowledge of a structure the less the need to resort to special methods of preparation for its recognition.

\section{IMBEDDING METHODS.}

\$ 47. When the consideration of time is not so important and finer results are more to be desired, the sections should be prepared 
according to some method in which an imbedding mass is used. The interstices of the tissue are completely filled with some substance that will give support and greater consistency and homogeneity to the tissue, and thereby enable the cutting of much thinner and more perfect sections.

There are three methods that are generally employed, $(a)$ the Paraffin method, (b) the Celloidin (Collodion) method, and (c) the Freezing method; the imbedding masses to fill the spaces being respectively paraffin, collodion and a congelation mass,--ice. The last is the simplest; it requires less expenditure of time, fewer reagents, and its results are in some ways the crudest. As in cutting free-hand sections without imbedding, the freezing microtome should be employed when haste is necessary and finer detail unimportant, as in clinical work. The two remaining methods may be employed in most cases and give good results. A choice between them must be determined by the special requirements of the case and a consideration of the differences of the two methods as set forth in tabular form below:

CELLOIDIN.

No heat required.

Sections relatively thick; 10 to $25 \mathrm{mi}$ crons or more.

Imbedding mass usually not removed.

Sections usually cut wet (with alcohol or oil).

Knife oblique.

Cutting stroke slow.

Form of the organ better preserved.

Imbedding requires more time.

Celloidin stains with basic dyes.

In general, better for larger specimens.
PARAFFIN.

Heat required.

Sections relatively thin; 1 to 10 microns or more.

Imbedding mass removed.

Sections cut dry.

Knife usually set at right angles.

Cutting stroke usually rapid.

Usually more or less distortion.

Imbedding requires less time.

$\S 48$. Despite the differences, the two methods may in most cases be used interchangeably. The advantages of the paraffin method are (1) the facility of its use and the ease with which thin sections may be obtained and its adaptation to serial sectioning. Celloidin is particularly useful when heal is injurious or the paraffin solvents (clearers) dissolve out substances which it is desired to preserve. Its main defect is the readiness with which the celloidin stains with basic stains, particularly such as Iron Hematoxylin and the coal-tar dyes (safranin, gentian violet, methylene blue, etc.) The celloidin may, however, be dissolved out. See $\S 136$.

$\S 49$. The following table will indicate the steps in the employment of the two methods: 
Living tissue

Fixing

$(\S \S 1-)$

Washing

Alcohols (\$ 9)

$(50 \%, 67 \%, 82 \%)<-\longrightarrow$ Staining in toto

$(\S 79)$

Dehydration

(95\%-99\% alcohol)

Paraffin Method

Celloidin Method

( Toluene, 1 part........ Clearing............Ether-alcohol

Abs. Alcohol, 1 part.

(1 hr. to 1 day)

Pure toluene,

(1 hr. to 1 day)

( Toluene, 1 part,

Paraffin, 1 part.

(2 hrs. to 2 days)

\{

$\downarrow$

Pure Paraffin

(2-to 24 hrs.)

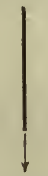

Imbedding paraffin, mass cooled.

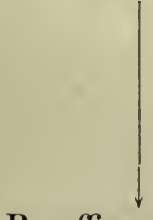

Paraffin sections (1-2 days)

Thin celloidin (2\% solution)

(1-14 days)

Thick celloidin (6\% solution)

(1-14 days)

Thick celloidin (12\% solution)

(1-14 days)

Celloidin mass

hardened in

chloroform

..... Imbedding. . . . . . .

Celloidin block clarified or placed in $82 \%$ alcohol cut............ Sectioning. ........... cut

Celloidin sections 
THE PARAFFIN METHOD.

$\S 50$. As seen by the above scheme, the aim is to fill all the interstices of the tissue with paraffin of the right degree of hardness to have it cut well. Paraffin is not soluble in water or alcohol, but is soluble in a number of fluids which in turn are miscible with alcohol. Hence the following steps are necessary: (1) the tissue must be first water-rid, thoroughly dehydrated with strong alcohol; (2) freed from the alcohol, cleared by a fluid that mixes with melted paraffin which (3) takes the place of the clearer in the tissue, infiltrates it, filling the spaces; (4) finally, the tissue is imbedded in paraffin of the right degree of hardness, the mass cooled, and it is ready (5) to cut, or section.

$\S 51$. Dehydration. After the various steps pertaining to the fixing and hardening ( $\S \S 7,9)$ of the tissue have been properly pursued it may be stored in alcohol of $82 \%$ to $95 \%$ strength depending on the tissue and its purpose. The dehydration necessary in imbedding may be accomplished by immersion in alcohol of $95 \%$ strength. For most work it is perhaps better to employ stronger (absolute) alcohol. If xylene or toluene are used for clearing absolute alcohol must be used.

Immerse small pieces 2 to $3 \mathrm{~mm}$. in diameter for at least 6 to 8 hours in $95 \%$ alcohol changed once or twice. A longer time, even days, usually does no harm and is preferable to ensure complete dehydration. For larger pieces of tissue or entire organs a correspondingly longer period of dehydration should be employed, a several days' stay, with the alcohol changed daily, being often advisable. In any case, dehydrate thoroughly, changing the alcohol 1 to 3 times, the last change usually being to absolute alcohol. Let the tissue dehydrate for a longer rather than a shorter period of time.

$\S 52$. Clearing. The alcohol must next be replaced by some solvent of paraffin which is miscible with alcohol,-a step spoken of as clearing. Toluene* is one of the most serviceable clearers, although for special purposes other media such as xylene, cedarwood oil, bergamot oil or chloroform may be preferred. Toluene (and xylene) mix well only with absolute alcohol, hence the dehydration needs to be thorough, and the clearing and infiltrating is best accomplished by a number of steps. After the absolute alcohol, the tissue is placed (1)

*Benzene, toluene, xylene, etc.; the terms adopted by American chemists. In Europe the same substances are designated xylol, benzol, etc. 
in equal parts of toluené and absolute alcohol for 1 hour to 1 day; (2) this is replaced by pure toluene for an equal period when the tissue will be clear and translucent,-except, of course, such as is dark in color.

Xylene may be used in place of toluene in nearly all cases. Steps 1 and 3 may often be omitted with very small objects. In some cases it is well to clear with cedarwood oil first ( $\$ 54)$ and then transfer to toluene (or xylene), toluene paraffin, etc. Familiarity with the tissue and the reagents will permit considerable departture from a fixed line of procedure.

$\S 53$. Infiltration. After the tissue is completely cleared in the toluene, remove it to a dish of (3) melted infiltration paraffin 1 part, toluene 1 part, and set it in a warm place (about $38^{\circ}$ C.), so that the paraffin may remain melted and the toluene slowly evaporate. After several hours or even days with very large pieces place the tissue in (4) pure paraffin in the paraffin oven for 2 to 24 hours, depending on the size of the piece. Quite large pieces may be left longer; with them one or two changes to fresh infiltration paraffin may be necessary. The melted paraffin replaces the toluene, filling in the interstices of the tissue. Paraffin melting at about $52-54^{\circ} \mathrm{C}$. is used. It is best not to expose to a higher temperature than is necessary, or for a long period of time as the heat tends to shrink and toughen, especially if the dehydration (and consequently the clearing) have been incomplete; this is particularly true of organs rich in connective tissue. The paraffin oven will be maintained at a temperature of $54-5^{\circ} \mathrm{C}$.

$\S 54$. Other Clearers. Cedarwood oil is a good clearer. It will clear from $95 \%$ alcohol if the dehydration has been carefully done. The tissue should be left in the cedarwood oil until it sinks and the alcohol currents have ceased to rise from it. The steps are given below. Thickened cedarwood oil such as is used for immersion objectives has been found to give excellent results with small objects and is to be recommended for some cell work. Chloroform gives excellent results but it penetrates (clears) slowly, so that it should be used only with small pieces of tissue. Chloroform or thickened cedarwood oil is to be employed with objects in which it is desired to preserve fat which has been blackened by osmic acid $(\S 227)$.

CHLOROFORM.

Alcohol (97-99\%).

Chloroform, until it sinks below the surface.

- Chloroform and paraffin, equal parts, for 4-48 hours, at incubator temperature (38 C.).

Pure paraffin, in the paraffin oven.
CEDARWOOD OIL.

$95 \%$ or absolute alcohol.

cedarwood oil.

Pure paraffin, in paraffin oven. 
$\S 55$. Imbedding. It is best to use fresh paraffin for imbedding and sometimes with a melting point higher than that of the infiltration paraffin, -52 to $54^{\circ} \mathrm{C}$. paraffin, answers well in a room of $19^{\circ}$ to $20^{\circ} \mathrm{C}$., and will be generally used. If the cutting is to be done in a room of lower temperature, a softer grade of paraffin may be used for imbedding; if at a higher temperature, a harder paraffin should be chosen, as when summer work is necessary.

As a general rule, hard tissues require a harder imbedding paraffin which is also better when very thin sections are desired. Large sections which usually must also be relatively thicker need a softer paraffin. It is better to work with a paraffin harder than the room temperature itself would call for and then regulate the cutting temperature by placing a source of heat such as an electric light nearer or farther away from the microtome knife.

Make a small paper box, fill it with the melted imbedding paraffin; float the box on a dish of cold water; transfer to it the tissue from the paraffin oven, arrange it carefully in the box in the way you wish it for cutting, and let the mass cool.

$\S 56$. In imbedding in paraffin observe the following rules: (1) Take no more paraffin (no larger box) than is needed to form a mass of convenient size around the specimen. The aim is to have as homogeneous a mass as possible; paraffin tends to crystallize if it cools slowly, hence the smaller the mass the more rapidly may it be cooled. (2) Let the imbedding paraffin when poured into the box be several degrees above its melting point, and the tissue likewise should have an equal temperature. Should the imbedding paraffin (or the tissue) be too cool it will not set well around the specimen, and a film of air may be enclosed. On the other hand, take care that the paraffin is not hot enough to "cook" the tissue, thereby shrinking it and rendering it hard and tough or ruining it altogether. (3) Cool the paraffin by floating the box on cold water. A homogeneous, translucent, paraffin mass can only be secured if it is quickly cooled. When a film has formed on the surface strong enough to resist rupture, immerse the block, or drop $95 \%$ alcohol upon the surface. Ice is an advantage in summer imbedding if cold water is not available. When ice or cold water are not available, good results have been secured by floating the box on a shallow dish of (used) ether-alcohol ( $\$ 63$ ). A homogeneous paraffin is only secured if the paraffin is allowed to shrink in cooling; it is therefore well to make the boxes as shallow as possible,- - that is, much broader 
and longer than high. Watch glasses, watch crystals, small tin pans, etc., may be used as imbedding receptacles.

$\S 57$. Crystallization of the imbedding mass. Paraffin that has crystallized is crumbly and will not give good sections. When crystallization has occurred, it is best to reimbed. Its occurrence is usually due to too slow a cooling of the imbedding mass, or cooling under conditions that prevent the paraffin from shrinking. It is sometimes due to the presence of impurities in the paraffin, such as water (?), excess of clearer, etc.

$\S 58$. Cutting the sections. The essentials for good paraffin sectioning are (1) well-imbedded tissue, (2) a sharp microtome knife (or section razor), ( $\$ 173$ ), (3) a room of the proper temperature, and (4) the paraffin block properly trimmed and arranged in the microtome. Furthermore, tissues fixed and hardened in different ways cut very differently. Tissue fixed in Hermann's, Flemming's, Müller's, Zenker's fluid or Carnoy's fluid, etc., cuts well; alcohol and mercuric chlorid tissue is more apt to be tough or hard, etc. The different organs and tissues have of course very different adaptabilities to the method.

After the imbedding mass is well cooled, remove the paper box and trim the part containing the tissue in a pyramidal form, two of the sides at least being as nearly parallel as possible. Clamp the block of paraffin in the holder of the microtome so that the tissue will be at the proper level for cutting, being careful to have the parallel sides also parallel to the edge of the knife. If a ribbon microtome is used, heat the holder and melt the end of the block upon it. Cool and place the holder in its place in the microtome, again having the parallel sides and the edge of the knife parallel. Use a very sharp, dry section knife for cutting the sections. Clamp it in the microtome slightly inclined to the cutting surface of the tissue. If the temperature of the room is right for the paraffin used, the sections will remain flat, and if the directions given above for trimming and arranging the block be observed, they will adhere and thus form a ribbon. If the room is too cold or the paraffin too hard, the sections will roll; if it is too warm, the sections will crush or be imperfect. If a microtome in which the knife is not fixed, is used, make the sections with a rapid straight cut as in planing. Do not try to section with a drawing cut as used in celloidin sectioning. $10 \mu$ will be found the most convenient thickness for the sections, though in special cases they should be thinner or even thicker. Handle the sections by means of a camel's hair brush, a needle, or sometimes on a scalpel handle, when cutting ribbons, etc. 
\$59. Difficulties in sectioning. (a) Rolling of the sections indicates too hard a paraffin, tissue or both, or too low a cutting temperature; bring the source of heat nearer or await better conditions. (b) Crushing together of the sections or wrinkling. Too soft a paraffin, too high a temperature, imperfect infiltration: alter conditions or reimbed, or reinfiltrate, etc. (c) The sections do not ribbon. Due usually either to (a) or to a failure to have the two sides of the block trimmed parallel and set parallel to knife edge. (d) The sections crack parallel to knife edge: tissue very brittle. (e) The sections are electric. The electricity is mainly due to friction in the cutting. The hardness of the tissue,in some cases due to presence of metallic salts, - or imperfection in the knife edge seem in many cases to be responsible; although in many instances the cause appears obscure. A tube of radium or a strong induction coil operating in the immediate neighborhood is said to obviate the difficulty.

Remember to have the paraffin block trimmed with two sides parallel and the knife edge parallel to these. Also, do not attempt to cut if the temperature of the room is too high,- -above $23^{\circ} \mathrm{C}$.

$\S 60$. Resumé of the method. To obtain as good results as possible with a certain organ fixed and hardened in a certain way, the steps must be carefully and exactly followed. Let the dehydration be complete, clearing thorough, infiltration sufficient; imbed, carefully observing the three cautions mentioned; and in cutting, remember to have a sharp knife, a cool room, and the imbedding block properly trimmed. Success also depends largely on the previous treatment in the fixer and on the care with which the fixer is washed out.

Properly employed, the paraffin method is widely serviceable, being only useless where the tissue is very large, very hard, hardened or injured by heat, or where the exact form of a large organ is important.

\section{THE CELLOIDIN METHOD.}

$\S 61$. A comparison with the paraffin method has already been given $(\S \S 47,48)$; there may be emphasized here three points: (1) with paraffin heat is required, with celloidin no heat; (2) paraffin must be removed from the sections subsequently, celloidin need not be and usually is not dissolved out; (3) by the paraffin method may be obtained small sections $(5 \mathrm{~cm}$. square or less), and thin, by the celloidin, larger sections, but thicker. With paraffin heat (melting and cooling) is necessary, and the mass is sometimes spoken of as a fusion imbedding mass; celloidin is a solution, and the mass is left in the tissues by exaporation. or its equivalent. 
In the celloidin method the imbedding mass with which the spaces of the tissue are to be filled is collodion, a solution of celloidin or proxylin* (soluble cotton) in ether and alcohol, hence the steps, which are comparable with those of the paraffin method (see $\S 47$ ), are (1) Dehydration, removal of the water; (2) Saturation with either-alcohol, the solvent of the celloidin; (3) Infiltration with celloidin solutions, a thin and a thick; (4) Imbedding in a thick celloidin mass, which is hardened and (5) sections cut.

$\S 62$. Dehydration. Let it be complete, as in the preparation for paraffin imbedding ( $\$ 51$ ). Immerse the tissue in $95 \%$ alcohol for 12 to 24 hours or longer, changing 1 to 3 times. Consult also $\S 51$ upon the dehydration of tissue.

$\S 63$. Saturation with ether-alcohol (equal parts of pure ether and absolute alcohol). Remove the tissue from the strong alcohol and place it in a stoppered vial of ether-alcohol for 12 to 24 hours. In addition to preparing the tissue for the collodion solutions, it completes the dehydration, should it be imperfect. In special cases, or if the dehydration is very thorough and the specimen small, this step may be omitted. A satisfactory infiltration is, however, more certain if ether-alcohol be used.

$\S 64$. Infiltration. (a) with thin celloidin. Pour off the ether-alcohol and add the thin (2\%) solution of celloidin in etheralcohol. This, being a solution in ether-alcohol with which the tissue is saturated, readily permeates it. It is best to allow at least a day for this to take place, although if there is time a stay of several days is better, there being little or no danger of deterioration while.in the solution. With large ( 1 c. c. + ) objects an infiltration of a week or even a month is advisable.

Infiltration. (b) with thick celloidin. Pour off the thin collodion solution and add thick ( 5 or $6 \%$ ) solution (in ether-alcohol). In this there is gradual concentration of the solution in the tissue. Allow small specimens to remain a day, or, better, several days;

*Celloidin is a specially prepared and purified form of pyroxylin. It is about twice as expensive as pyroxylin or soluble cotton but with it better solutions can be prepared. The pyroxylin on the market seldom affords stronger solutions than $8 \%$; with celloidin a $12 \%$ solution is easily prepared. The trimmings from the celloidin blocks (after the alcohol or chloroform hardening) may be dried out. and redissolved and thus used over and over again. Pyroxylin may be used equally well. 
larger objects should be given a proportionately longer time, a week to a month, or even longer.

If the object to be imbedded, such as many embryological specimens, is one with large interior cavities with thin walls the transfer from the thin solution to the thick solution may be attended by a collapse of the walls and a consequent shriveling and distortion of the specimen. Aroid this by allowing the thin solution to thicken very gradually by evaporation in a dry atmosphere, as under a belljar with calcium chlorid present until the solution has attained the right consistency. To accomplish this it is only necessary to have the cork of the rial containing the specimen perforated by a small hole. A small piece of paper may be inserted with the cork, or with porous corks no special effort need be made. Unless the thick solution has itself thickened by evaporation, with large specimens it is advisable to follow the $6 \%$ bath with a stay in a thicker solution, as 10 or $12 \%$, for a day or so.

$\S 65$. Imbedding. Pour off the $6 \%$ or $12 \%$ solution and add for a short time at least a $12 \%$ solution of celloidin (in ether-alcohol). The tissue is now ready for imbedding in $12 \%$, which may be accomplished in either of two ways: (a) on a holder or (b) in a paper box. Only those specimens need be imbedded in a box that, from their shape, or for purposes of careful orientation or serial sectioning, require a larger imbedding mass around them.

(a) On a holder (wooden-block). Choose a block of a convenient size; put a drop or two of celloidin upon one end and insert a pin vertically to the surface near the edge. Transfer the tissue from the vial of thick celloidin to the block and lean it against the pin. The shape of many tissues will obviate the need of a pin. Pour the thick celloidin onto the tissue, drop by drop, moving the block in such a way that the thick viscid mass may be made to surround and envelop the tissue. Continue to add drops of celloidin at intervals until the tissue is well surrounded, and then as soon as a slight film hardens on the surface invert the holder bearing the tissue in a shell-vial of large diameter or glass box containing enough chloroform to cover the specimen. Cork or cover so that the chloroform will not evaporate. If the piece of tissue is of awkward size and shape, oiled paper may be wound around the end of the wooden holder and tightly tied, the projecting hollow cylinder being long enough to receive the object. The tissue may be put into the cylinder as before, the celloidin slowly poured in drop by drop until the specimen is 
completely covered. When a film has formed, place in chloroform as before.

(b) In a paper box. When a box is required for imbedding proceed as follows: The inside of the paper box should be slightly oily to prevent the celloidin from sticking to it. Rub upon the paper that is to be folded to form the box a little vaseline, and then with a cloth or lens paper remove as much as possible. Fold the paper into a box of convenient size and shape. Remove the object from the thick celloidin and place it in the box, arranging it in the manner wished with a view to sectioning it later. Pour over it slowly, drop by drop or a little at a time, a $12 \%$ solution of celloidin until the specimen is well covered and the box sufficiently filled. It is better to have a deep layer over the specimen. The $12 \%$ solution does not afford the best mass for cutting, so that, with large objects, it is better to allow the mass in the box to thicken by evaporating it slowly under a bell-jar (aquarium jar) until it has attained such a consistency that it is no longer fluid.

$\S 66$. Hardening. When the celloidin mass is thick enough so that it only dents when touched with the finger nail it is ready for hardening. This may be done by pouring chloroform into the jar in which the imbedded material is placed, covering from the air. The chloroform vapor hardens the mass. When it is well set it may be transferred to a jar of the chloroform which takes out the etheralcohol and hardens the celloidin mass, for which a few hours is sufficient. Allow the chloroform to act for 6 to 24 hours. The imbedding mass remains quite transparent when no water is present. The hardening action of the chloroform may be quickened and intensified by carefully heating the chloroform until bubbles of ether begin to come from the specimen. Do not let the chloroform evaporate.

$\S 67$. Alcohol hardening. When the celloidin mass is hard, whether clear or not, it may either be transferred to alcohol of about $82 \%$ strength in which it is stored until cut, or it may be placed in Clarifier (castor oil, 1 part; xylene, 3 or 4 parts). Alcohol of higher percentage softens the mass; lower grades such as $67 \%$ usually increase the hardness of the celloidin and in some cases are to be recommended.

The choice between alcohol and clarifier involves no decision of importance in technique. The method of clarification has the advantage that the orientation of the specimen in the microtome preparatory to cutting can be more perfectly 
done. If the tissue has been stained in toto $(\S \S 79,142)$ the sections may be mounted directly from the clarifier as soon as cut. Any mercuric chlorid precipitate $(\S 11)$ that may be present can be dissolved out by means of a solution of iodin in the castor-xylene. The castor oil, however, renders the microtome, knife, hands of the operator, etc., sticky and the method is not so cleanly as the alcohol method. On the other hand, alcohol tends to rust microtome and knife. Clarification is preferred by the writer for serial work in celloidin.

§ 68. Clarification. Celloidin blocks transferred from the chloroform hardener to an oil mixture such as castor-xylene ( $\$ 67)$ will become quite transparent (clarified) and hardly discernible, so that the tissue is readily seen. Sometimes, however, the celloidin remains white and opaque, due to the presence of moisture, and considerable time is required for its clarification. In such cases the process may be hastened by placing the tissue in the clarifier in a warm place, and changing the clarifier sereral times. If the block still remains opaque, remove to $9 \tilde{5} \%$ alcohol for a day for dehydration, pass through chloroform, and into clarifier. In this way the mass may usually be cleared perfectly. Change the clarifier to fresh after the first day or so. The sectioning may be done after a few hours' immersion, although a several days' clarification is preferable.*

$\S 69$. Cutting the Sections. There is no marked difference in the sectioning of celloidin blocks preserved in alcohol and those that have been clarified. In the following paragraphs $67 \%$ alcohol should in the reading be substituted for clarifier if alcohol was used in the hardening.

If a paper box was used, after the celloidin is ready for cutting, remove the paper, trim the block as is desired (see below), put some thick celloidin upon the wooden block or other holder and press the base of the celloidin block firmly against it; within two minutes it will be firmly cemented and one may proceed at once to clamp the holder in the microtome and commence cutting.

For celloidin sectioning, a long drawing cut is necessary in order to obtain thin, perfect sections. The knife should, therefore, be set at an obliquity of 15 to $20^{\circ}$ or less, so that half or more of the

*The imbedded object may remain in the castor-xylene clarifier indefinitely without harm. The celloidin grows somewhat tougher by a prolonged stay in it. After cutting all the sections desired at one time, the imbedded tissue is returned to the clarifier for future sectioning. It should be remembered, however, that pure castor oil is a solvent of celloidin, hence it is necessary to have the container tightly stoppered, otherwise the volatile xylene will evaporate leaving the castor oil behind. 
blade is used in cutting the section. Recall that in the paraffin method the knife is usually to be set at right angles to the direction of the cut, and the stroke is a rapid straight one. Trim away the surrounding celloidin mass leaving enough, however, to serve as a support to the tissue and prevent its bending under the impact of the knife; if the celloidin mass is too tapering, bending will occur and thin sections cannot be cut. To avoid this the celloidin block is best trimmed in the form of a four sided truncated pyramid with as broad a base as possible.

Clamp in the jaws of the microtome, placing it so that the mass of celloidin is opposite the side to which the pressure of the knife is applied in cutting. It is advantageous also to have the object placed with its long diameter parallel with the edge of the knife.

When knife and tissue are properly arranged wet the tissue well with clarifier or alcohol,- as the case may be,- and flood the knife with the same. Make the sections with a slow, steady, motion of the knife. With a small object $(3 \times 5 \mathrm{~mm}$.) and a good sharp knife, sections $5 \mu$ to $6 \mu$ can be cut without difficulty. In addition to a sharp knife, however, there are necessary well-infiltrated tissue and a hard, firm mass. If serial sections are not desired, it may be more expeditious to cut dry and with a rapid stroke.

$\S 70$. Transferring the sections. If the sections are quite thick they may be transferred from the knife to a slide or a dish by means of forceps or a brush; if they are thin, however, it is better to handle them by means of an absorbent tissue paper, as follows: Flood the sections well with clarifier and then by means of a pipette remove the clarifier from the knife and place over the sections the end of a piece of the tissue paper, pressing it down upon the sections if necessary. Carefully pull the paper off the edge of the knife; the sections will adhere to the paper. Place the paper, sections down, on a slide, taking care that the sections are in the desired position. With the finger carefully press the sections (through the transfer paper) to the slide, and then lift the paper, with a rolling motion, from the slide; the sections will adhere to the slide. Should they stick to the paper instead, lower the paper again and again firmly press the section to the slide. For further procedure see $\S \S 135,136$. If it is not desired to mount the sections upon a slide immediately, or if they are to be kept in bulk, as for class work, the transfer paper may be shaken gently in a dish of clarifier 
or $95 \%$ alcohol and the section (or sections) will float free and sink to the bottom.

$\S 71$. Serial Sectioning. If it is desired to mount the sections in series, proceed as follows: With a.camel's hair brush or needle draw the first section, when cut, up toward the back of the knife and make the next section. Place this section to the right of the first, and so on, arranging them in serial order, section after section, and line below line, until enough are cut to fill the area that the cover-glass will cover. Flood the sections as before by letting the clarifier flow over them, being careful, howerer, not to float them from their places. Absorb the clarifier from the knife with a pipette, and place over the sections a piece of the transfer paper twice the width of a slide; press it down if necessary, and slowly draw it off the edge of the knife. Should it then be seen that some of the sections are adhering to the knife instead of the paper, it means that the clarifier had been allowed to thicken* on them, cementing them to the knife, and the preliminary flooding to insure their being free, was insufficient. In that case it is best to flood the paper with clarifier, carefully lift it, arrange the sections again, flood them with clarifier, place a clean piece of transfer paper over them and try again. One soon becomes accustomed to the behavior of the sections, and accidents are rare. In cutting a series of many small sections, some time is consumed and it is necessary to flood the sections on the knife frequently with clarifier while cutting in order to prevent the clarifier thickening and cementing them to the knife.

$\S 72$. Resume of the method. Success in the employment of the celloidin or collodion method depends upon the thorough infiltration with the solutions, requiring days or even months, and the employment of a thick imbedding mass giving when hardened a firm unyielding support to the tissue. This may be gained by employing a relatively long period of infiltration, and taking pains in imbedding to have the imbedding mass well thickened.

Observing these two cautions, celloidin may be used in almost all cases as an imbedding mass, except such as are affected by the conditions of the methods already mentioned ( $\S 47$ and 61 ).

*If one is a long time cutting a series of sections, it sometimes occurs that the xylene evaporates leaving the castor oil that is thick and viscid and also a solvent of the collodion, so that the sections are not easily transferable but stick rather firmly to the knife. In such a case, fresh clarifier or even a little xylene to dissolve the castor oil must be used. 
THE FREEZING METHOD.

§ 73. This method is expeditious and of use in the rapid examination of tissues, and therefore especially serviceable in the pathological laboratory and in clinical diagnoses. It may also be used in cutting tissues that are too hard to be cut satisfactorily by means of either the collodion or paraffin methods, and in the examination of tissues for substances (e. g., fats) which the solutions necessary for the paraffin and celloidin methods dissolve out. Both fresh and fixed tissue may be cut by means of the freezing microtome and with or without any special mass such as is used in paraffin or celloidin imbedding. Some histologists quite prefer the freezing method to the paraffin or celloidin methods for general use.

When no mass is employed the tissue is simply frozen and cut, or, if it is fixed tissue, soaked well in water first and then frozen. When extreme haste is not so essential it is better to first saturate the tissue with some solution that does not crystallize on freezing, but simply hardens, since the formation of the ice crystals is hurtful to the tissue. Such are solutions of gum arabic or sugar and aniseseed oil, and they are spoken of as Congelation masses.

§ 74. Infiltration. Gum arabic or anise-seed oil may be used. (a) Gum arabic. If the tissue has been fixed and is in alcohol remove the alcohol by soaking it for several hours to 1 day in water. Remove to a thick solution of gum arabic in water, in which it may remain for about 24 hours. It is then ready to freeze and cut.

(b) Anise-seed oil. For this method the tissue should be first dehydrated $(\S 51) . *$ When dehydration is complete, transfer the tissue to anise-seed oil, in which it may soak for 12 to 24 hours; it is then ready to freeze and cut. It is particularly adapted for use with tissue that has been stained in toto.

$\S 75$. Cutting. Place a drop of the solution of gum arabic (or anise-seed oil) upon the object carrier of the freezing microtome and turn on the carbonic acid (or ether) spray. When the mixture begins to harden, place the object upon it in an abundance of the solution and freeze it nearly solid. Covering with an inverted cup hastens the freezing. An especially wedge-shaped knife is necessary because of the hardness of the mass.

*Anise-seed oil will, however, clear from $90 \%$ or even $82 \%$ alcohol; this is sometimes of advantage. 
When the tissue is completely frozen, cut it with a straight movement of the knife, as in the paraffin method, holding it firmly upon the knife rest and making the strokes as rapidly as possible, at the same time rapidly raising the tissue a few microns at a time by means of the microtome screw. There are a number of automatic microtomes specially designed for use with the freezing method.

The mass of sections is transferred to a dish of water in which the gum arabic is dissolved away and the sections are ready for staining $(\S \S 137,146)$. If anise-seed oil is used, the sections are to be transferred to $95 \%$ alcohol which will dissolve out the oil; if the tissue has been stained in toto the sections may be transferred to anise-seed oil (or other clearer) and mounted in balsam directly.

$\S 76$. Rapid Method. Blocks of tissue 1 centimeter thick should fix in $10 \%$ formalin 12 to 24 hours. If haste is a factor, take thinner pieces and fix for 1 minute or more. Trim the block so that it is about $5 \mathrm{~mm}$. thick; rinse in water for a few seconds, transfer to the freezing microtome, freeze and section.

Float the sections when cut from the knife into water from which they may be gotten upon the slides by means of a camel's hair brush. Drain off the water and press the sections out smooth by means of blotting paper, filter paper or other absorbent paper. Cautiously drop over the sections $\mathbf{9 5 \%}$ and absolute alcohol and follow this immediately with thin $(3 / 4$ or $1 / 4 \%)$ celloidin solution ( $\$ 64$ ) which when it has partially evaporated out will serve to support the section and-fasten it to the slide. It is now ready for staining. (§ 144).

\section{STAINING.}

$\S 77$. Staining has for its first and primary purpose, the rendering outlines and structures more distinct by giving them a color contrast with their surroundings (color image). A second and more important use is for the differentiation of particular structures or substances which by their selective staining facilitate the histological analysis. Rational staining, like rational fixation, depends upon the physics and chemistry of staining reactions; indeed, in the demonstration of particular substances the fixation and staining should be determined by the mutual interdependence of their reactions, since they have the same purpose,- - the preservation and demonstration of the substance sought for. 
In some cases the differential staining may be accomplished in the fixing (fats, impregnations). Differential staining, histo-chemical methods, micro-chemical methods (as applied to the animal organism) thus belong in the same category and rest upon a physico-chemical basis. As in the case of fixation, a great deal remains to be done in the perfection of this side of histological technique.

$\S 78$. Classification of Stains. Stains may be grouped: (a) according to their chemical composition as (1) organic;--hematoxylin stains, carmine stains, anilin stains, (coal-tar dyes; benzene derivatives), and (2) inorganic. (b) From another chemical aspect as (1) basic or (2) acid, depending upon the chemical reaction of the staining principle or group, (3) neutral (\$85). (c) Histologically, stains are: (1) nuclear (chromatin stains), (2) plasma or general stains, (3) special stains, (4) impregnations. The first are usually basic, the second acid, the fourth inorganic.

$\S 79$. In toto staining. When in toto or bulk staining is employed, the piece of tissue is stained entire and imbedded and sectioned afterwards. In this case the tissue should be stained before the process of embedding has begun, after the washing out of the fixer has been completed $(\S \S 7,9)$. But a single stain may be given and the one chosen is generally a nuclear one,--hematoxylin, cochineal, or carmine ( $\$ 91,98-)$. A counter stain may be given subsequently after sectioning, orange $\mathrm{G}$ after hematoxylin or cochineal, Lyon's blue after carmine being most satisfactory. In toto staining is particularly useful in serial sections of embryological material and in morphological work in general, as it saves time and manipulation.

$\S 80$. Section Staining. The application of the staining processes after the tissue is imbedded and sectioned. This is more serviceable, especially if highly differential results are desired.

$\S 81$. Progressive and Regressive Staining. According to the mode of application, staining is either progressive or regressive. Progressive staining consists in permitting the staining to proceed to the right degree of intensity and then stopping it. In regressive staining the tissue is over-stained and the excess of stain removed by the application of a Differentiator. Uusally in the regressive method there is employéd a Mordant which is a chemical solution serving to make the stain "take." It possesses a double affinity,to the tissue and to the stain, which is usually made to operate in the interest of differential or selective staining. The mordant is not 
always in the form of a separate solution (e. g., aluminium hematoxylin) and in many cases the mordantage is given in the fixing. Mordantage, directly or indirectly employed, is also useful in staining by the progressive method. Stains or dyes that do not require a mordant are termed Substantive, those requiring a mordant to make them "take" are Adjective stains.

Delicate results in exact differential staining by either the progressive or regressive methods can best be secured only by section staining, although differentiation of the sections after in toto staining may be resorted to.

$\S$ 82. Differentiation. In the regressive method it is necessary to remove the excess of stain by the application of a solution that will usually differentiate it, or bring out the selective action of the stain. A small amount of differentiation is usually necessary in any case for the most delicate results. Alcohol, $95 \%$ or other grade, may often be used as a differentiator. $95 \%$ acidified with hydrochloric acid $(1 / 10$ to $1 \%$ ) is sometimes used, or a special differentiator is required (acetone, alum solution, clove-oil, etc.). It is usually necessary to control the differentiation by use of the microscope.

$\S 83$. Impregnations. In addition to the typical methods of coloring tissue by means of stains there is a group of methods in which the coloring matter is deposited in the cell or tissue that it is desired to differentiate, in the form of a precipitate. These are known as impregnation methods and are of great value, especially as applied to nervous tissues. A hard and fast line, however, cannot be drawn between true staining methods and impregnations. Silver nitrate and gold chlorid are the substances most generally employed in the impregnation of tissue $(\$ \S 221,223)$.

$\S 84$. Choice of stains. Remember that the staining is greatly affected by the previous treatment, brilliancy or selectivity in the result being in many cases defendent on the fixer employed or impaired by the improper or incomplete washing out of the fixer.

In staining, therefore, consider three things, $(a)$ what it is desired to bring out,- -what kirds of stains you need to employ, (b) the mode of fixation that has been employed, and $(c)$ the imbedding method must also be considered in the choice, since if celloidin is employed certain stains that color it deeply should be avoided. 
(a) For general purposes it is customary to use a nuclear and a cytoplasmic stain either in combination or successively. The double staining most employed is hematoxylin and eosin. Triple stains are generally not so satisfactory; hematoxylin and picrofuchsin is one commonly used. Neutral stains $(\$ 85)$ are essentially triple stains. Quadruple stains are rarely serviceable; see, however, $\S 129$. For stains to be employed for special purposes, see Special Methods, § 174-.

(b) In the case of most of the fixers given $\S 11-$, there are no restrictions as to the stains that may be employed though some are more satisfactory than others. With Flemming's fluid and Hermann's fluid, however, it is well to employ only such stains as iron hematoxylin ( $\$ 94)$ and anilin stains, - safranin being suggested as a red stain, gentian violet as a blue stain. Dichromate fixers whose action has been prolonged usually require strong stains. Iron hematoxylin and similar stains may be used after any fixer. The EhrlichBiondi-Haidenhain stain ( $\$ 111)$ is most satisfactory after Mercuric chlorid ( $\$ 11)$.

(c) Celloidin is deeply stained by such stains as iron hematoxylin, safranin, methylene blue, gentian violet, and basic stains in general. If it is necessary to use these, with celloidin material, the celloidin should be removed from the sections before mounting (§ 136).

$\S 85$. Neutral Stains. In the case of most of the anilin stains, if an aqueous solution of a basic stain is added to an aqueous solution of an acid stain, there is formed by combination a neutral stain which is usually but slightly soluble in water and hence precipitates out. Neutral stains are however moderately soluble in strong methyl or ethyl alcohol. These facts it is well to keep in mind in using anilin stains. The reaction may be made use of for increasing the selectivity, sharpness and color tones of the original stains. Neutral stains may be used in one of three ways: (a) The neutral stain is often soluble in excess of the acid or basic stain or in an aqueous solution of another acid or basic stain (§111). (b) Dissolved in methyl (or ethyl) alcohol and diluted nearly to the precipitation point the activity and selectivity of the component stains seem to become specially marked $(\S 128,214)$. (c) The formation of the neutral stain may be carried out in the staining process itself,- - "on the slide,"-by staining first strongly with an aqueous solution of the acid stain, rinsing away the excess stain and staining with the aqueous solution of the basic stain $(\S 106)$ and differentiating $(\S 82)$ with alcohol, or alcohol and clove oil.

Of the large number of stains that combine to form neutral stains may be mentioned, - the acid stain being given first; - eosin and methylene blue ( $\$ 128)$; orange G. and gentian violet ( $\$ 131$ ), erythrosin and toluidin blue, thiazin red and toluidin blue, coerulein S. and safranin, acid violet and safranin. These may be combined and used in any one of the three ways mentioned above. 
$\S 86$. The time of staining. Although in general certain time limits can be given to the period during which a stain should be allowed to act, with most stains, especially those with which no differentiation is needed, such as hematoxylin, and most carmines, the correct intensity of color should be determined by examining the preparation with the microscope. One soon becomes able to judge of the right stain in this way better than if a given time were adhered to.

In the use of stains requiring a subsequent differentiation, the rule is to over-stain and watch the differentiation carefully with the microscope, stopping it when sufficient. In this case it is the differentiation and not the staining that should be carefully regulated. In general, for the best results, it is advisable to use staining and differentiating solutions in dilute form and prolong the time during which they act.

The following formulas include the more generally useful stains and those to be employed in the "Special Methods" given subsequently.

STAINS.

$\S 87$. Hematoxylin Stains. Hematoxylin is a colorless compound of acid properties forming therefore salts with bases which oxidize readily forming "hemateates." The oxidation product of hematoxylin is hematein which is the real staining principle and may in some cases be used with real advantage instead of hematoxylin. Hematoxylin (hematein) itself has little value as an acid (plasma) stain; combined with metallic bases it becomes a valuable basic (chromatin) stain. The metals usually employed as mordants for hematoxylin are: aluminium, iron, copper, chromium, molybdenum, vanadium. Their salts may be used either in the same solution as the hematoxylin (aluminium, molybdenum, vanadium) or separately (iron, copper.) Solutions of the same metals may in some instances (aluminium, iron, chromium,) be employed also as differentiator. For preparing hematoxylin stains it is a great convenience to have a $10 \%$ stock solution in $95 \%$ alcohol.

$\S 88$. Chloral Hematoxylin. [11] Formula: Potassium alum, 8 grams; distilled water, 250 c. c.; hematoxylin $2 / 10$ th gram or 2 c. c. hematoxylin stock solution. Boil 5 or 10 minutes in an agate dish. After cooling add 6 grams of chloral hydrate. Place in a bottle and permit the hematoxylin to oxidize for a week or two, or 1 to $2 \mathrm{c}$. c. hydrogen peroxid may be added. Its staining quality improves up to an optimum and then begins to deteriorate. Old hematoxylin generally contains a precipitate and should be filtered often or before using.

Stain sections 5 to 30 minutes according to the age of the solution, the character of the tissue and the fixation employed. After staining wash well with distilled or tap water. Usually no differentiation is required unless a purer chromatin stain is desired $(\$ 175)$. Counter stain as desired. 
\$ 89. Mayer's Haemalum. Formula: haematein, 1 gram; $90 \%(95 \%)$ alcohol, 50 c. c.; potash alum, 50 grams; distilled water, 1,000 c. c. Dissolve the haematein in the alcohol, the alum in the water; mix.

This is an excellent formula, giving a good stain immediately after it is made up and retaining its selective staining quality for a year or longer. It is one of the best hematoxylins for ordinary work.

For this formula, Mayer has now substituted the following: hematoxylin, 1 gram (10 cc. stock solution); distilled water, 1,000 c. c.; sodium iodate, 0.2 grams; potassium alum, 50 grams. The hematoxylin is first dissolved in the water and then the other ingredients are added. Dissolve and filter. 50 grams of chloral hydrate and 1 gram of citric or acetic acid may be added as a preservative. Stain as given in $\S 88$. In using it is frequently advisable to dilute one or more times with distilled water.

§90. Ehrlich's acid hematoxylin: Formula: Water, 100 c. c.; $95 \%$ alcohol, 100 c. c. hematoxylin crystals, 2 grams ( 20 c. c. stock solution); dissolve in the alcohol; glycerin, 100 c. c.; glacial acetic acid, 10 c. c.; alum in excess. Let the mixture ripen in the light until a dark red. Sections stain in this hematoxylin in a short time, generally 5 to 10 minutes. Wash with water after staining.

$\S 91$. Delafield's hematoxylin. Formula: Saturated aqueous solution of ammonia alum, 200 c. c.; hematoxylin stock solution 20 c. c. Allow the mixture to stand in the light and air in an unstoppered bottle for 4 or 5 days; filter and add glycerin, 50 c. c., and methyl alcohol, 50 c. c. Permit it to stand for a week or so to ripen; filter and keep in a stoppered bottle. The staining power increases for several months. In using, dilute 3 or 4 times or more with distilled water. It is useful for in toto staining diluted 1:9 with $20 \%$ alcohol or distilled water.

Stain sections from water; 4 to 5 minutes will generally be sufficient. Wash well with water after staining. In toto staining 1 to 14 days may be necessary depending upon the size of the object.

This is a very strong hematoxylin stain and may be used to advantage with tissues that stain with difficulty. It is likewise a more diffuse stain than either chloral or Ehrlich's hematoxylin, staining cell-body as well as nucleus, - a feature having its advantages. Old solutions (several months to a year) should be filtered before using.

§ 92. Acid Hematoxylin (Delafield's). The dilution of Delafield's hematoxylin ten to twenty times with water, and making slightly acid with acetic gives a useful stain particularly for differentiating nuclei (embryological work.)

§ 93. Muchematein (Mayer). Aqeuous formula: Rub up $0.2 \mathrm{grm}$. hematein with a few drops of glycerin; add $0.1 \mathrm{grm}$. aluminium chlorid, $40 \mathrm{c}$. c. glycerin, 60 c. c. distilled water. Filter if necessary. Alcoholic formula: Hematein, 0.2 grms.; aluminium chlorid, 0.1 grm.; $70 \%(67 \%)$ alcohol, 100 c. c.; 1 or 2 drops nitric acid. Formulae with a minimum amount of aluminium designed as specific stain for mucus. The aqueous solution is more selective; the alcoholic formula designed for the staining of mucus that swells excessively in water ( $\$ 234)$.

§94. Iron Hematoxylin (Heidenhain). Formula: (a) Mordant; $2 \%$ aqueous solution of ferric alum (iron-ammonium-persulphate). (b) Stain; $\mathrm{I} / 2 \%$ aqueous solution of hematoxylin ( $10 \%$ alcoholic stock solution, 5 c. c.; 
distilled water, 95 c. c.). (c) Differentiator; the ferric alum mordant, preferably diluted several times. An excellent stain, especially for cytological work. It may be used after any fixer.

The steps necessary are: (1) Mordanting 1 to 24 hours; (2) rinse the sections in water 10 to 30 minutes; (3) stain for 3 to 24 hours; differentiate slowly and control it under the microscope. The slides may be alternately dipped into the ferric alum solution for a few seconds and then into tap water. (4) Wash in running water 15 to 60 minutes. The ferric alum mordant may be used several times as well as the hematoxylin solution whose staining quality improves up to a limit by use.

§ 95. Copper Hematoxylin (Weigert). Formula: Mordant; $3.5 \%$ aqueous solution of copper acetate; Stain; $10 \%$ alcoholic solution of hematoxylin 10 c. c.; distilled water, 90 c. c.; saturated solution lithium carbonate, 1 c. c. Differentiator; potassium ferricyanide, 2.5 grms., borax, 2 grms.; distilled water, 200 c. c. Designed for staining the myelinic sheath of medullated nerve fibers ( $\$ 199)$ but useful for other purposes.

The steps are: (1) mordanting for 1 to 24 hours, (2) rinsing the sections in water 10 to 30 minutes, (3) staining for 3 to 24 hours, (4) differentiate slowly controlling the action under the microscope. (5) Wash in running water 30 minutes or longer. The mordantage may be given in bulk if desired. As usually employed with tissue fixed in chrome fixers, the stain is strictly a chrome-copper hematoxylin. Other differentiators may be used if desired. Mount in neutral balsam.

§ 96. For Mallory's phospho-molybdic hematoxylin, Heidenhain's vanadium hematoxylin, and other hematoxylin formulae of occasional usefulness, consult the works on technique (p. 90).

\$ 97. Carmine Stains: Like hematoxylin, the carmine stains depend upon an acid staining principle,-carminic acid,-which in combination with bases gives a red nuclear stain of value. The metals usually employed in carmine formulae are aluminium, calcium, strontium, iron. Carmine itself is (Liebermann) a combination of carminic acid with aluminium, calcium, and protein. It is soluble in acids and alkalis. The necessary mordant is not so often employed in a separate solution as is the case with hematoxylin. See, however, iron carmine $[6,30]$.

§ 98. Carmalum (Mayer's) Formula: Carminic acid, 1 grm.; potassium alum, 10 grms.; distilled water, 200 c. c. Dissolve with heat (if necessary). Filter. Add 1 c. c. formalin as a preservative. It may be used for in toto or for section staining.

Stain sections 5 to 30 minutes or as long as necessary. Rinse tissue before staining with distilled (not tap-) water.

$\S 99$. Borax carmine. (Grenacher). Formula: Borax 4 grams; carmine, 3 grams; water, 100 c. c.; allow the mixture to stand for several days, shaking occasionally when most of the carmine will have dissolved; filter and add 100 c. c. of $70 \%$ alcohol. Let the mixture remain for several days, filter again and the solution is ready for use.

This is a good carmine stain for in toto staining. Stain objects in toto for one to several days, according to size; remove to $67 \%(70 \%)$ alcohol, acidulated 
slightly with hydrochloric acid, (4 drops in each 100 c. c.), for a day and then remove to $80 \%$ alcohol. It affords a bright red stain that is quite transparent.

$\S 100$. Paracarmine (Mayer). Formula: Carminic acid, 1 gram; aluminium chlorid, 0.5 gram; calcium chlorid, 4 grams; $70 \%$ (67\%) alcohol, 100 c. c. Allow it to stand a day or so, shaking occasionally until the carminic acid has quite dissolved, and then filter.

This is an excellent carmine stain for in toto staining. The tissue may be stained one to several days (1 week), then washed in $67 \%$ and $82 \%$ alcohols to remove the excess of staining fluid. A red nuclear stain, more opaque than borax carmine. It does not over-stain readily, and since it is an alcoholic solution $(70 \%)$ it is quite penetrating and may be allowed to act for a greater length of time, being thus suited for staining in toto objects of considerable size.

$\S 101$. Hcl. carmine. Formula: Carmine, 2 grams; concentrated hydrochloric acid, 3 c. c.; $70 \%$ alcohol, 100 c. c. Boil gently for 15 to 20 minutes to dissolve the carmine; cool and filter.

This is a strong carmine stain, quite suitable for sections, especially such as stain with difficulty. It may also be employed for staining in toto. Stain sections from alcohol or water for 5 to $\mathbf{1 5}$ minutes; rinse away the superfluous stain with $67 \%(70 \%)$ alcohol and differentiate for a few seconds to a minute with acid alcohol $(95 \%$ alcohol 100 c. c., concentrated hydrochloric acid $1 / 10$ c. c.). Wash away the acid alcohol with ordinary $95 \%$ alcohol. If a pure nuclear stain is not desired the differentiation may be omitted.

Picric acid ( $\$ 116)$ may be used as a counter stain, and in that case differentiation is ordinarily not required.

$\S 102$. Alum Cochineal. Formula: Powdered cochineal, 75 grams; potassium alum, 75 grams; distilled water, 1000 c. c. Boil the ingredients for half an hour, or (better) macerate for a day or so, boiling up two or three times. Cool and filter. Add to the filtrate distilled water to make up 1000 c.c. and a crystal of thymol as a preservative.

This is an excellent stain, particularly for in toto staining of embryos, giving a purpler stain than the carmine stains given above.

Stain objects, such as embryos, over night to 2 days or longer, depending on size. Wash out with water, 2 to 6 hours and place successively in $50 \%, 67 \%$ and $82 \%$ alcohols several hours to a day in each.

$\S$ 103. Mucicarmine. Formula: Carmine, 1. gram; aluminium chlorid (pure), 0.5 grams; distilled water, 2 c. c. Mix thoroughly together and heat over a small flame for 2 minutes (in a test tube) until the mixture has become dark red. Dissolve the whole in 100 c. c. $50 \%$ alcohol added gradually; after 24 hours filter. For use, it may be diluted 5 or 10 times with water.

Stain sections 10 minutes or longer in the diluted stain, rinse in water, dehydrate, clear, and mount in neutral balsam.

If desired, the stain may be diluted with 50 or $67 \%$ alcohol instead of water.

$\S 104$. Gentian Violet. Formula: A concentrated solution in distilled water. Stain (paraffin) sections from water for 5 to 10 minutes, rinse in water, dehydrate and differentiate with $95 \%$ alcohol and complete the differentiation with clove oil. When the differentiation is sufficient, clear with bergamot oil 
and mount in balsam. This may be used alone to give a blue stain with tissue fixed in Hermann's or Flemming's fluid. (See also $\$ 112,131$ ).

$\$ 105$. Methylene Blue. This valuable stain, used particularly in the histology and pathology of the blood and nervous system, and in bacteriology, is represented in a large number of formulae. For the staining of nuclei, basic granules in the cytoplasm, neurochromatin granules, etc., simple aqueous solutions may be employed. A $1 \%$ solution suffices for most purposes; in some cases, a concentrated solution is to be preferred.

Stain from water for 5 minutes to as many hours, with or without heat, rinse with distilled water, differentiate if desired in a $1 / 10$ th $\% \mathrm{Hcl}$. in $95 \%$ alcohol, or $1 / 10$ th $\%$ alum solution. Wash, dehydrate, mount in neutral balsam.

$\S 106$. Alkaline Methylene Blue. Formula: Methylene blue, 2 grms.; absolute or $95 \%$ alcohol neutralized with pure dry sodium carbonate, 50 c. c.; add distilled water, 450 c. c.; $1 \%$ potassium hydroxid, 5 c. c. An excellent stain, giving best results after mercuric chlorid fixers (incl. Zenker's, etc.).

$\$ 107$. Eosin-Methylene Blue. Stain sections $1 / 2$ hour with a $1 / 2 \%$ to $1 \%$ aqueous solution of eosin, rinse in water, stain in alkaline methylene blue 10 minutes, rinse well in water. Differentiate and dehydrate rapidly with neutral $95 \%$ alcohol and absolute alcohol, clear in xylene, mount in neutral balsam. Particularly useful for staining blood in the tissues (hemolymph) glands, etc..

$\S 108$. Toluidin Blue. This may be used, often to advantage, in place of and for the same purposes as methylene blue. It gives a somewhat darker stain.

$\S 109$. Methyl green. This is a nuclear stain of much value, besides being an important ingredient of triple stains (e. g., Ehrlich's triacid mixture and $\S 111$ ). In very dilute solutions it is serviceable in staining the nuclei of fresh tissue and of isolated cells. A $1 \%$ aqueous solution may be used with hematoxylin and picrofuchsin in differentiating the structure of the hair follicle. (Gage).

$\$ 110$. Safranin. Formula (Babe's): Concentrated aqueous solution of safranin, 1 part; concentrated alcoholic solution of safranin, 1 part.

Stain sections 1 to 4 hours, or over night; wash away excess of stain with $95 \%$ alcohol, differentiate with acid alcohol (95\% alcohol, 100 c. c., hydrochloric acid, $1 / 10$ c. c.) for a few seconds, rinse with $95 \%$ alcohol and clear in carbolxylene or bergamot oil. If a pure nuclear stain is not required, the differentiation may be omitted. This gives a good stain with tissue fixed in Hermann's or Flemming's fluid. It is a brilliant, transparent red.

Other formulas may be employed (concentrated alcoholic solution, alcoholic solution diluted with anilin water, equal parts concentrated solutions in alcohol and anilin water, etc.). Differentiation may be accomplished with the use of iodin-potassium iodid solution, or by counterstaining with an alcoholic solution of light green or acid violet $(\S \S 121,122)$.

§ 111. Ehrich-Biondi-Heidenhain Mixture. Formula: Saturated aqueous solutions of Orange G. Rubin S. (Fuchsin acid) and Methyl green, 100 c. c., 20 c. c. and $50 \mathrm{c.}$ c. respectively. In preparing the mixture, only fully saturated solutions should be taken which should be mixed in the order given slowly with constant agitation. Only tissue that has been fixed in sublimate solutions ( $\$ 11$ ) should be used, the sections should be thin and slightly acid. This may be secured by 
treating with $1 / 10$ th per cent. acetic acid before staining. The stain is useful for some cytological work (nuclear degenerations, cytoplasmic transformations, etc.).

In staining use the stock solution diluted with distilled water 1:60 and rendered slightly acid by the addition of $0.2 \%$ acetic acid, drop by drop, until the red color tone, due to the fuchsin acid, becomes slightly accentuated. The success of the stain depends upon having the staining reaction right; if too acid, the fuchsin acid predominates, otherwise the green and orange prevail. Stain 6 hours or more; when sufficient, dehydrate rapidly with absolute alcohol, clear with xylene, mount in xylene balsam.

$\S 112$. Flemming's Triple Stain. Stain sections in an alcoholic solution of safranin diluted with an equal volume of anilin water, for a day or longer. Differentiate in absolute (or $95 \%$ ) alcohol with $1 / 10$ th $\% \mathrm{Hcl}$ until hardly any more color comes away. Stain for 1 to 3 hours in a $1 \%$ aqueous solution of gentian violet. Rinse in distilled water and treat with a strong $(2 \%)$ solution of Orange G. in distilled water, and while clouds of violet are still being given off, bring the sections into absolute alcohol in which the differentiation is begun. Transfer the sections to clove oil or bergamot oil which completes the differentiation and clears. Mount in balsam before the last pale clouds of color have ceased to come away.

This stain is only recommended after such fixers as Flemming's fluid or Hermann's fluid. The stain is somewhat fickle, giving the best results only after some practice. It is useful in some cytological work.

Several modifications have been proposed. A short method is frequently employed as follows: (1) stain for a second or two in a mixture of equal parts of saturated aqueous and saturated alcoholic solutions of safranin; (2) rinse in water; (3) stain 2 to 10 minutes in $1 \%$ gentian violet; (4) rinse in water; - and (5) stain for 10 seconds or longer in a $2 \%$ solution of orange G. . Dehydrate rapidly with absolute alcohol; clear and differentiate.with clove oil, controlling the differentiation under the microscope. Remove the clove oil with xylene or toluene and mount in balsam.

$\S 113$. Congo Red. A well known indicator, red in neutral or alkaline solutions, turning blue in the presence of free mineral and many organic acids, not affected by acetic, lactic or carbonic acid in the presence of ammonia. It is useful as a plasma stain after hematoxylin, gentian violet, etc.

It may be employed in aqueous $(1 / 2 \%)$ or alcoholic $(2 \%)$ solution. With subsequent differentiation in acid alcohol $(\S 110)$ it is a useful stain with gastric glands. Occasionally useful as an indicator with living organisms or tissue.

$\S 114$. Eosin. Formulas: (a) $1 / 4 \%$ aqueous solution; (b) $2 \%$ aqueous solution; (c) $1 / 10 \%$ solution in water or $95 \%$ alcohol. Formula (a) is preferable for most work; (b) affords a stronger and (c) a weaker stain. This may be used as a counter-stain with hematoxylin to differentiate nucleus from cell-body. Stain sections after hematoxylin for 10 to 30 seconds, wash away the excess of stain with distilled water or $67 \%$ alcohol: Since alcohol tends to wash out the eosin, unless the color is too strong it is advisable to hasten the process of washing out and dehydration.

$\S 115$. Erythrosin. Formulas: (a) $1 / 2$ to $1 \%$ solution in $67 \%$ alcohol, (b) $1 / 2$ to $1 \%$ aqueous solution. This is a general stain similar to eosin in its 
staining properties, but gives a redder color. Formulas (a) and (b) may be used with sections and in the same way as eosin.

$\S 116$. Picric Acid. A counter-stain useful after carmine or hematoxylin. Use a $1 / 2$ to $1 \%$ solution in $67 \%$ or $95 \%$ alcohol, or simply add it to the alcohol used in dehydration. It washes out the hematoxylin and is useful as a differentiator of this stain, with which it is well to overstain somewhat if it is desired to counterstain with picric acid.

$\S 117$. Orange G. An excellent acid stain that may be employed as counterstain and differentiator after hematoxylin, gentian violet, etc.

Employ (a) 2 to $4 \%$ aqueous solution which may with advantage be slightly acid ( $\mathrm{Hcl}$.$) ; in the last event, sections should be rinsed well before the mounting.$ It may with good effect be combined with eosin, erythrosin or fuchsin acid. (b) Frequently a concentrated solution in $95 \%$ alcohol is to be preferred to the aqueous solution.

$\S$ 118. Fuchsin Acid (Rubin S., Magenta S.). Like Congo red, an indicator, red in acid or neutral solutions, bleached by alkali. It is a valuable plasma stain, but requires care in its use because of its sensitiveness to alkali. Tap water should therefore be avoided. The staining solution and the mounting medium should preferably be slightly acid,-at least not alkaline.

Employ a 2 to $4 \%$ solution; stain 1 minute to 24 hours.

$\S 119$. Picro-fuchsin. Formulas: (a) General stain, $-1 \%$ aqueous solution of fuchsin acid, 10 c. c.; saturated aqueous solution of picric acid, 75 c. c.; distilled water, 25 c. c. (b) For nervous tissue, $-1 \%$ aqueous solution of fuchsin acid, 15 c. c.; saturated aqueous solution of picric acid, 50 c. c.; distilled water, 50 c. c. This is a valuable counter-stain to hematoxylin, especially serviceable in the differentiation of white connective tissue fibers. The nuclei are a purplish brown (hematoxylin stain), the connective tissue red, cell bodies and muscle yellow-orange. In special cases the relative amount of fuchsin acid may be decreased or increased, thus giving a preponderance to the yellow or red in the general stain.

Stain well with hematoxylin, rinse in water, and stain with the picrofuchsin 15 to 30 seconds; wash away the excess of stain with distilled water or $67 \%$ alcohol. Picro-fuchsin will gradually wash out the hematoxylin, therefore stain strongly with hematoxylin and regulate carefully the time of staining with picrofuchsin. Picro-fuchsin is quite sensitive to alkalies, so that tap-water (unless slightly acidulated) should not be used for washing out and the mounting medium should be slightly acid or neutral, not alkaline.

$\S 120$. Mallory's Anilin Blue Connective Tissue Stain. Fix tissue in Zenker's fluid (preferred) or in a mercuric chlorid fixer; imbed preferably in paraffin.

Sections are to be stained (a) for 5 minutes or longer in a $1 / 5 \%$ aqueous solution of acid fuchsin, and (b) without washing, stained about four times as long in the following: Grübler's water soluble anilin blue, 0.5 gram; orange G., 2.0 grams; $1 \%$ aqueous solution of phosphomolybdic acid. 100 c. c. Wash, differentiate and dehydrate with $95 \%$ and absolute alcohol. Clear in xylene and mount in balsam. 
A stain of quite general usefulness. The collaginous connective tissue fibers, reticular tissue, cartilage, osseous tissue, mucus, etc., are stained blue; nuclei, cytoplasm, muscle, red; red blood corpuscles, orange.

$\S 121$. Light Green. An acid stain sometimes useful as a counterstain with safranin. A $0.2 \%$ alcoholic solution may be used in the differentiation of the latter stain.

$\S 122$. Acid Violet may be used in similar cases and in the same manner.

$\S 123$. Bleu de Lyon (Lyons' Blue). A plasma stain frequently useful as a counter stain after safranin or carmine (in toto). Employ an alcoholic $(95 \%)$ solution of about half saturation and stain sections for 10 to 15 minutes or longer. Rinse with $95 \%$ alcohol and dehydrate more or less rapidly depending on the differentiation desired.

$\S 124$. Iodine. In addition to a certain value as a fixer and to facilitate the removal of mercuric chlorid from tissue, usefulness as a differentiator after basic aniline dyes, iodine has a place among the stains for the differential coloring of starch, glycogen, amyloid, cellulose, etc. The following solutions may be mentioned: (a) saturated aqueous solution; (b) Iodine-potassium iodid solutions. (Gram's, Lugols), -iodine 1 gram, potassium iodid 2 grams, distilled water 300 c. c Lugol's solution is six times as strong. (c) $10 \%$ alcoholic solution, (tincture); (d) for a formula useful for staining glycogen, amyloid, etc., see $\S 229$.

$\S 125$. Resorcinfuchsin (Weigert). Prepare a concentrated solution of the dry powder in a $1 \%$ solution of hydrochloric acid in $95 \%$ alcohol. Designed for staining differentially elastic connective tissue fibers.

Stain sections 15 to 30 minutes, wash and dehydrate in $95 \%$ and absolute alcohol, clear in xylene, mount in balsam.

The stain may be directly prepared according to a modified formula of Weigert, Formula: Fuchsin (basic), 2 grams; resorcin, 4 grams; water, 200 c. c. Boil several minutes (10 or more); add 25 c. c. $30 \%$ solution ferric chlorid and boil 5 or 10 minutes longer. If the stain is not all precipitated, more of the ferric chlorid solution may be added. Permit the liquid to cool. Let the precipitate settle and decant fluid, or filter. Dissolve precipitate in 200 c. c. $95 \%$ alcohol, employing heat if desired (boiling on a water bath). Filter. When the filtrate is cool, add 4 c. c. strong hydrochloric acid.

The stain may be followed by picro-fuchsin or other similar reagent for staining the white connective tissue fibers.

$\S 126$. Orcein (Taenzer-Unna). Another standard method of staining the elastic connective tissue fibers (elastin). Formula: Orcein D., 1 gram; $95 \%$ alcohol, 100 c. c.; strong hydrochloric acid, 1 c. c.

Stain sections $1 / 2$ to 1 hour, wash and differentiate in $95 \%$ alcohol and acid alcohol ( $\$ 82)$. Elastin stained dark brown. Other differential stains may be used on the same preparation.

$\S 127$. Verhoeff's Elastin stain. [44] Formula: Hematoxylin, 0.15 grams; Absolute alcohol, 25 c. c.; dissolve by heat and add 1 drop of $5 \%$ ammonia solution. Permit it to stand for at least 5 minutes and add Lugol's solution ( $\$ 124)$, 22 c. c.; filter and permit it to stand for 24 hours in a corked bottle. The solution remains good for about 3 months. For use, add a $7 \%$ alc. sol. ferric chlorid, 1 drop per c. c. 
Stain sections from alcohol, 12 to 24 hours; wash in water 1 to 5 minutes; differentiate in a $1 \%$ ferric chlorid solution (alc. stock solution, 1 part, water 6 parts). Counter stain with picric acid ( $\$ 116)$. A sharp stain, though possibly not in all cases completely differential.

128. Jenner's Stain [27]. This is an eosinate of methylene blue. Formula: Mix equal parts of $1.25 \%$ eosin (water soluble) and $1 \%$ methylene blue. Permit the mixture to stand for 24 hours, then filter, wash the precipitate on the filter with distilled water, dry it and dissolve it in methyl alcohol $(0.5 \mathrm{grm}$. precipitate in 100 c. c. alcohol).

This is primarily a stain for blood films. Sections may be stained in one of two ways: (a) Stain 1 to 5 minutes, dilute the stain with an equal volume of water and continue the staining for 5 to 10 minutes. Rinse with distilled water, differentiate and dehydrate rapidly with $95 \%$ and absolute alcohol. Clear in xylene. Mount in balsam.

(b) Dilute the stain with 1 to 4 volumes of distilled water and stain 1 to 24 hours. Further treatment as above. To insure permanency, it is well to thoroughly remove the alcohol with xylene in clearing, by 2 or 3 treatments.

$\S 129$. Wright's stain. Contains several staining principles, notably eosinates of methylene blue, methylene azure, in solution in methyl alcohol, and is made according to a complicated method, for which see original article [46].

Primarily for blood films ( $\$ 214$ ). Dissolve 0.2 grams of the dry stain in 100 c. c. of methyl alcohol. In staining sections follow the procedure given in $\$ 128$.

$\S 130$. Nochts-Hastings Stain [22]. Similar in its composition to Wright's stain and used in a similar manner. In staining sections, follow the procedure given in $\S 128$. Dissolve 0.3 grams of the dry stain in 100 c. c. of methyl alcohol. An excellent blood stain $(\$ 214)$.

$\S 131$. Neutral Gentian Violet (Reinke, Bensley). Formula: Saturated aqueous solution of gentian violet and saturated solution of orange G. are mixed in equal proportions and permitted to stand 24 hours. The precipitate that forms is removed by filtration, washed with distilled water, dried, and a concentrated solution in methyl or ethyl alcohol is made. When ready to use it, dilute 1 to 4 times with distilled water. Stain 12 to 24 hours, drain away the stain, dehydrate rapidly with $95 \%$ and absolute alcohol, continuing the differentiation if necessary with oil of cloves, to the right degree. Remove the oil of cloves with xylene (thoroughly) and mount in balsam.

\section{PREPARATION FOR STAINING.}

$\S$ 132. After the sections are cut and before the process of staining can begin, certain steps are necessary, such as the removal of the paraffin in the case of paraffin sections. Furthermore, it is usually advisable to fasten the delicate sections to the glass slides on which they are to be finally mounted before beginning the series of manipulations that are necessary in Staining and Mounting. To consider the latter first: 
$\S 133$. Handling the Sections. Sections may be carried on through the staining and mounting processes either (a) not fastened to the slide, - as free or loose sections, or, (b) fastened to the slide, which is of the greatest advantage and practically necessary in the case of serial sections. The methods of fastening the sections to the slide are different for celloidin and paraffin sections.

$\S 134$. Free sections. It is seldom necessary or advantageous to carry paraffin sections through the processes of staining and mounting not attached to slides because- of their delicacy and the readiness with which they tear or fray. Celloidin sections may be more conveniently carried on in this way. Loose sections may be carried on in watchglasses or larger glass vessels if there are many of them, the sections either being transferred from vessel to ressel by means of forceps or a section lifter, or the fluid decanted, care being taken not to pour off the sections, and the succeeding medium added. Single sections may be best carried on upon the slide which must be kept horizontal. When the fluid is to be changed, place a brush or needle gently on one corner of the specimen and pour off the liquid, if necessary first absorbing most of it by means of a pipette; in this way the section may be retained on the slide. If many sections, not in series, are to be treated in the same manner, they may be placed in a perforated container,-box or basket,and handled as a unit by transferring the container from fluid to fluid ( $\S \S 144-)$. When the final step is reached $(\$ 159)$ the sections may be transferred to slides and mounted.

$\S 135$. Fastening sections to the slide. 1. Celloidin sections. Sections cut by either the alcohol or the clarifier methods may be conveniently fastened to the slide in the following manner:

If the sections are transferred to the slide from clarifier or clearer, absorb the fluid thoroughly by placing over the section some absorbent paper and pressing it down gently and firmly, repeating the operation several times with fresh paper. After the oil is well absorbed, with a pipette drop upon the section enough ether-alcohol to thoroughly wet it. This softens or dissolves the celloidin and on its evaporation the section sticks to the slide. Allow the ether-alcohol to evaporate until the celloidin has again set and the surface of the section looks dull or glazed and then place it in a jar of $95 \%$ alcohol. Do not let the sections dry.

If the sections are in series, it is better to put the ether-alcohol on one end of the slide and let it run quickly over the sections and 
drain from the other end of the slide, repeating the operation two or three times if necessary. If the sections are well pressed down and the clarifier thoroughly absorbed the sections will stick to the slide under most rough manipulation. If, however, it is found that the sections tend to float off of the slide in the process of staining, their adhesion may be insured by using albuminized slides, or removing the slides from the alcohol ( $\$ 144)$ and again treating with etheralcohol.

Sections cut in alcohol may require more ether-alcohol.

$\S 136$. Celloidin Sections with Removal of the Celloidin. In the above method, the celloidin is used in fastening the section to the slide. As celloidin stains heavily with most basic stains, it is sometimes desirable to have it removed as is paraffin. In most such cases, the difficulty may be avoided by employing paraffin as the imbedding method; where this is not possible, imbed in celloidin as usual but harden and cut in alcohol. Use $67 \%$ alcohol on the microtome knife, handle the sections with tissue paper as usual ( $\$ 70$ ), but before transferring them to slide previously prepared with albumen fixative ( $\$ 138$ ), let as much of the alcohol evaporate as is possible without the sections drying. They should also be free from wrinkles. Press the sections down well, remove the tissue paper and place the slide in $\mathbf{9 5 \%}$ alcohol which will harden the albumen fixative cement. The celloidin may be removed now or just before clearing by means of ether-alcohol, absolute alcohol, or clove oil.

$\S 137$. Free-hand Sections and such made with the freezing microtome may be fastened to the slide if desired by albumin fixative or $3 / 4 \%$ celloidin or both. If the first is used, the sections should be transferred to the albuminized slide from water which should be largely absorbed and the sections pressed down by tissue paper. If the latter, press sections to a clean slide with paper, pour on $95 \%$ alcohol, dip cautiously into $3 / 4 \%$ celloidin, drain and place slide in 95\% alcohol.

$\S 138$. 2. Paraffin Sections are most conveniently fastened to the slide by albumen fixative and spreading. with water, as follows:-Place upon the slide a small drop of Mayer's albumin fixative* and with a (clean) finger tip spread it into a thin even film. Place upon the albuminized surface enough distilled water to float

$M^{*}$ ayer's Albumen fixative. White of egg, 50 c. c.; glycerin, 50 c. c.; salicylate of soda, 1 gram. Shake them thoroughly and filter. 
the sections freely. Cautiously and slowly warm the slide over a small flame, as that of an alcohol lamp, or upon a metal warming table until the sections begin to spread and straighten out. When the wrinkles have entirely disappeared, allow the water to cool and then drain it off, retaining the sections in position. The slide should now stand 2 to 3 hours or better over night when the water beneath the sections having evaporated, they will have been brought close to the albumen fixative. They are now ready for the remaining steps. Melt the paraffin by warming the slide over a low flame and place it in xylene $(\S \S 143-)$.

$\S 139$. Albumin fixative and Heat. If the sections are free from wrinkles or with few wrinkles that can be easily "ironed out," place the sections in position upon a slide prepared with albumin fixative as above, and with a clean finger press the section into the albumin fixative beginning at one edge of the section and by a rolling motion of the finger, ironing out any wrinkles that there may be. It is well to look upon the reverse side of the slide to see if the section really adheres to the albumen fixative, as in some cases it does not. Heat the slide gently and slowly over a small flame until the paraffin melts and begins to run away from the specimen. Keep the paraffin just melted for a minute or so, and then transfer to the xylene. Should the paraffin section not adhere to the albumin fixative when well pressed down, it can in many cases be made to do so by briskly rubbing the reverse side of the slide with a woolen or silk cloth.

$\S 140.3 / 4 \%$ Celloidin (collodion). The adhesion of sections that are particularly valuable or relatively thick may be ensured by treating them with $3 / 4 \%$ celloidin, as follows: Fasten the sections to the slide by either of the above methods, remove the paraffin by xylene $(\$ 143)$, and then after draining off the xylene from the slide, 10 to 13 seconds, it is put into a bottle containing $3 / 4 \%$ celloidin. In a minute or more the celloidin displaces the xylene and penetrates the sections. The slide is removed, allowed to drain for half a minute and then put into a jar of $67 \%$ alcohol which sets the celloidin [17]. It is now ready for the staining processes ( $\$ 144-)$.

\section{PRELIMINARY STEPS.}

$\S 141$. These are somewhat different for paraffin and celloidin sections. In the case of the former, it is necessary to remove the paraffin by means of a solvent (e. g., xylene), remove the paraffin 
solvent by alcohol, and usually remove the alcohol with water. In the case of celloidin sections the first step is unnecessary. If the tissue was hardened and cut in alcohol, the second step may likewise be omitted.

$\S$ 142. In toto Staining. If the staining has already been done, $(\$ 79)$ these "preliminary steps" are of course all unnecessary, save the removal of the paraffin by xylene.

$\S 143$. Xylene. Leave paraffin sections in xylene until the paraffin is entirely dissolved out, requiring usually only a few seconds. A longer stay generally does no harm.

$\S 144$. Alcohol. Transfer paraffin sections from xylene to $95 \%$ alcohol leaving the sections in the alcohol 5 to 10 minutes; or if you wish, shorten the period to a minute or so by waving the slide gently to and fro in the alcohol.

Celloidin sections cut by the clarification method, are placed in alcohol to remove the clarifier. This may take a longer time, and if there are many slides it is well to use two changes of alcohol. A longer stay in alcohol does no harm.

$\S 145$. Water. Remove the $95 \%$ alcohol with water if the stain is an aqueous one.

$\S 146$. Staining. The following schema shows the general steps in staining and mounting. In all the processes, seemingly complicated, if it is remembered that the succession of media as in histological technique generally depends upon their miscibility or some special reaction, and the reason for the various steps is recognized, much of the difficulty in remembering the order in which they come will be avoided. 
SCHEMA FOR THE STAINING OF SECTIONS.

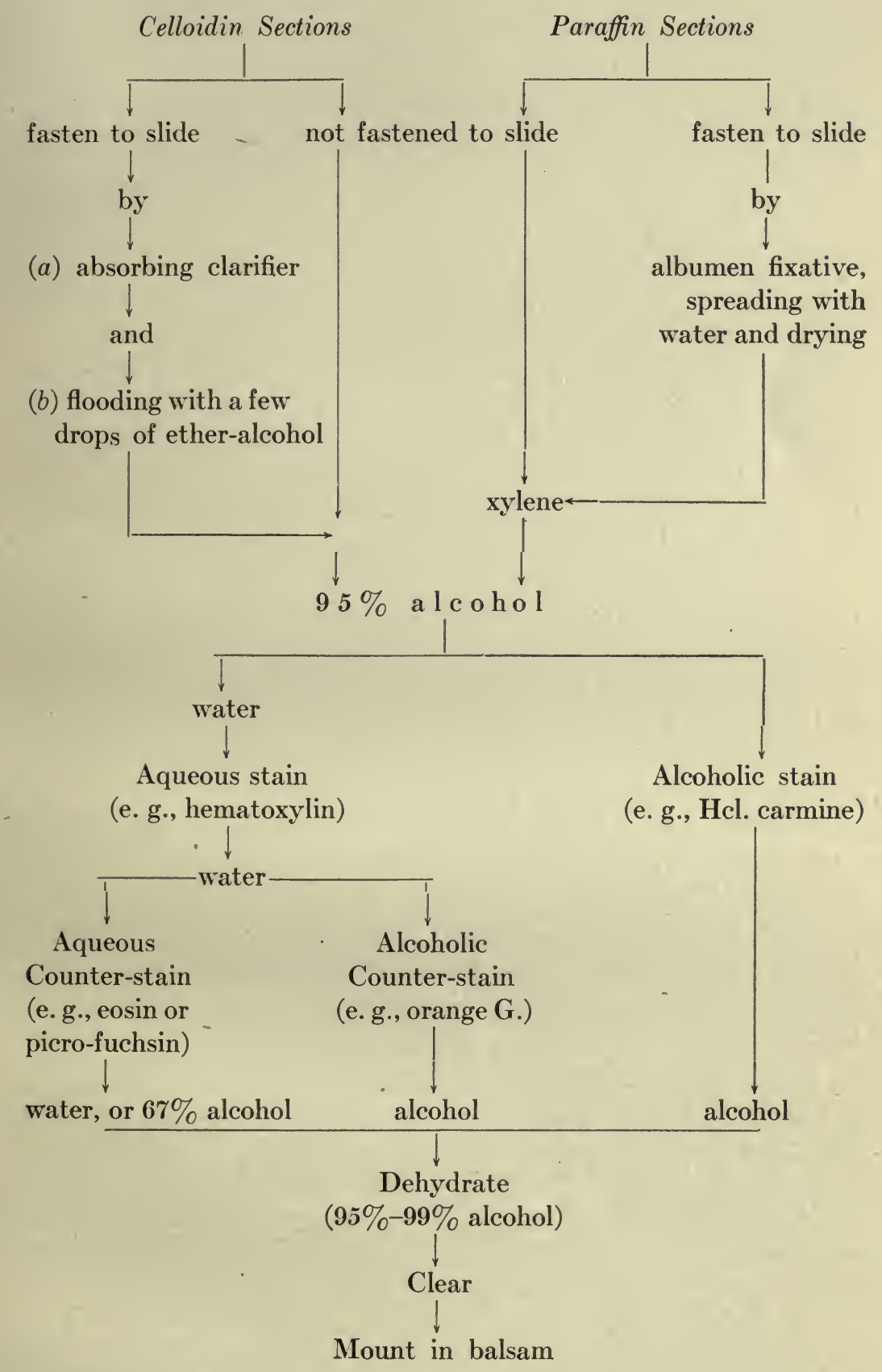




\section{MOUNTING.}

$\S 147$. Whether stained or unstained, prepared for microscopical examination by isolation or sectioning, and especially if it is desired to keep the preparation, it is necessary to mount it in some way, -i.e., so arrange it upon some suitable support (glass slide) and in some suitable mounting medium that it may be satisfactorily studied with the microscope.

Mounting may be

I. Temporary, or

II. Permanent, - as

A. Dry, or in air,

B. In a medium miscible with water, or

C. In a resinous medium, in which case it is necessary first to remove all water by either $(a)$ drying-Desiccation, or (b) a series of displacements, $i$. e., 1 . Removing the water with strong alcohol-Dehydration; 2. Removing the alcohol with clearerClearing; 3. Replacing the clearer with balsam or other resinous mounting medium.

$\S 148$. Temporary mounting. Illustrations may be found in the examination of blood corpuscles and living ciliated cells ( $\$ 35$ ). Temporary examination of tissues is quite simple, though important, and for this it is only necessary to place the teased tissue or section on the slide in a drop of the fluid in which it is at the time, normal salt solution, dissociator, or alcohol, and cover. The examination of preparations intended for permanent mounts during the staining or before mounting will often serve to detect faulty treatment at a time when it may be remedied without great expenditure of time, or to discard the specimen as worthless.

$\S 149$. Permanent mounting. These usually include $(a)$ mounting dry on a ring or in a cell, $(b)$ in glycerin or glycerin jelly, media miscible with water, and $(c)$ in Canada balsam or damar, resinous media.

$\S 150$. Mounting dry. The preparation, may be either upon the under side of the cover-glass (best if possible) or rest upon the bottom of the cell.

In the first case a shallow cell made by a shellac ring will be sufficient; in the second, a shellac ring may not give a deep enough cell and a paper, hard rubber, or metal ring may be cemented to the slide. 
(a) When the preparation is on the cover. Prepare a shellac cell ( $\S 156)$ on the slide of a size slightly smaller than the cover to be used, and allow it to dry for a day or so. Warm the cover bearing the preparation to remove the last traces of moisture, and place it film side down upon the ring. Warm the slide until the edge of the cover may be made to adhere to the shellac ring and press the cover down until it adheres all the way round. Seal the cover with shellac and label $(\S \S 163,165)$.

(b) Mounting in a paper or rubber cell. With a brush, cover one side of the ring with a layer of shellac and place it on the center of the slide, shellac side down; place within the cell the preparation, arranging it in the manner desired, and place upon the ring a cover-glass of a suitable size, and seal it with shellac; label.

$\S 151$. Mounting in glycerin media. (a) Pure glycerin; glycerin and acetic acid, $1 \%$; (c) glycerin and a stain. As glycerin extracts most stains (Ex. Carmine) it is sometimes advisable to have a small amount of stain dissolved in the glycerin used for mounting. Of such combined mounting and staining mixtures may be mentioned (1) Glycerin and Congo red, (2) Glycerin and carmine. Other combinations may be used.

$\S 152$. Congo Glycerin. Formula: $1 / 2 \%$ aqueous solution, Congo Red, 1 part; glycerin, 1 part.

$\S 153$. Carmine Glycerin. Formula:-Carmalum, 25 c. c., Glycerin, 75 c. c.

$\S 154$. Methyl green and Eosin Glycerin. Formula:-1\% aqueous solution methyl green, 2 c. c.; $1 / 2 \%$ aqueous eosin, 1 c. c., glycerin, 97 c. c.

Glycerin and glycerin-jelly are most serviceable in mounting isolation preparations. For both of these mounting media the object must be mounted from water or an aqueous solution.

Arrange the section or teased tissue in the center of the slide, drain off the water or aqueous solution in which the preparation is and add a small drop of glycerin. Take a clean cover in the forceps, breathe on the under side and carefully lower it upon the object; gently press it down. It is best to use only a small drop of glycerin so as not to get it outside the cover, as it is hard to clean away satisfactorily. Clean carefully and seal with shellac in accordance with § 163. 
$\S 155$. Mounting in glycerin-jelly. The preparation should be mounted from some aqueous solution. Warm the slide gently and put it upon the centering card; in the center of the slide place a drop of warmed (melted) glycerin-jelly. Remove the object from the water or aqueous solution and arrange it in the glycerin-jelly. Grasp a cover-glass with the fine forceps, breathe on the lower side, gradually lower it upon the object and gently press it down. Allow the glycerin-jelly to set, keeping the slide horizontal meanwhile. Scrape away the superfluous glycerin-jelly around the cover-glass and seal with shellac ( $\S 163)$.

$\S 156$. Preparation of shellac mounting cells. Place the slide upon the turn-table and center it (i. e., get the center of the slide over the center of the turn-table). Select a guide ring on the turntable which is a little smaller than the cover-glass to be used; take the brush from the shellac, being sure that there is not enough cement adhering to it to drop. Whirl the turn-table and hold the brush lightly on the slide just over the guide ring selected. An even ring of the cement should result. If it is uneven, the cement is too thick or too thin or too much was on the brush. After a ring is thus prepared, remove the slide and allow the cement to dry spontaneously, or heat the slide in some way. Before the slide is used for mounting, the cement should be so dry when it is cold that it does not dent when the finger nail is applied. A cell of considerable depth may be made with shellac by adding successive layers as the previous one dries.

$\S 157$. Mounting in balsam: by desiccation. Certain preparations may be mounted in balsam, by drying, the method of desiccation ( $\$ 147$ ), e. g., cover-glass preparations of bacteria, stained cover-glass preparations of blood, etc. For this is it only necessary that the preparation be absolutely dry, a small drop of balsam placed upon it or upon the under side of the cover-glass, which is carefully placed over the specimen and pressed down.

Mounting in balsam: by displacement. Mounting in balsam by desiccation is serviceable for but few preparations in histology, and in most cases the removal of the water by a series of displacements is resorted to $(\S 147)$. For this the following steps are necessary: Dehydration, Clearing, Mounting in balsam.

$\S 158$. Dehydration. The sections are entirely freed from water by the use of $\mathbf{9 5 \%}$ or absolute alcohol. The slide or freesec- 
tion may either be placed in a jar of alcohol or alcohol from a pipette be poured over it. Treat the preparation to be mounted for 5 to 15 minutes. The thicker the section the longer the time required; celloidin sections require a longer time than paraffin sections. In any case, be sure that the dehydration is complete, giving a longer rather than a shorter time, and then clear.

$\S 159$. Clearing. This is accomplished by putting the slide in a jar of clearer or dropping the clearer upon the section from a pipette. When the section is cleared it will be transparent. Test it by holding it against a dark background; if it is not cleared it will be cloudy, white, and opaque. Carbol-xylene (melted carbolic acid, 1 part; xylene, 3 parts); xylene; or certain essential oils (origanum, thyme, cajuput, bergamot) are used.

$\$ 160$. Mounting in balsam. Drain off the clearer and allow the section to stand until there appears the first sign of dullness from evaporation of the clearer from the surface. Then place a small drop of balsam upon the section or upon the cover-glass which is then inverted over the specimen.

Remember that in mounting in this way you must always " $D e$ hydrate, Clear and Mount in Balsam," and that the three steps are inseparable.

$\S 161$. Natural balsam is acid in reaction due to organic acids contained. As these bleach basic dyes, notably hematoxylin, it is well to use for most purposes balsam that has been dried out and redissolved in a known solvent, such as xylene or benzene. Neutral balsam solutions are to be preferred. Alkaline balsam is sometimes preferable for some hematoxylin stains; acid balsam in certain other cases (fuchsin acid, injection masses, § §118, 217-). Furthermore, according to Mann [36], such solvents as xylene readily oxidize with the formation of acid products. For delicate work, therefore, it is probably advisable to use as thick balsam as is convenient, and avoid inclusion of air bubbles in the mounting. Benzene balsam from this standpoint is preferable to xylene balsam.

Certain resinous mounting media,-Camsal Balsam, Euparal,- have been recently prepared in which specimens may be mounted direct from $95 \%$ alcohol; clearing is then unnecessary, of course.

\section{SEALING THE PREPARATIONS.}

$\S 162$. Sealing glycerin mounted specimens. Wipe away the superfluous glycerin as carefully as possible with a moist cloth or a piece of lens paper. Place four minute drops of cement carefully at the edge of the cover at the four quarters and allow them to harden for half an hour or more; these will anchor the cover-glass and the 
preparation may then be placed upon the turn-table and a ring of shellac cement put round the edge while revolving the turn-table.

$\S 163$. Sealing glycerin-jelly mounts. Allow the glycerinjelly to harden for 12 hours or longer. With a knife scrape away the superfluous jelly and then carefully wipe around the cover-glass with a cloth moistened with water. Place the slide on a turn-table, carefully center the cover-glass, and with a brush seal the edge of the cover by a ring of shellac while revolving the turn-table. A second coating may be given subsequently if needed, after the first coat has dried.

$\S 164$. Sealing balsam mounts. This is necessary only with special preparations, and should in any case be done only after the preparations have dried out for several weeks. With a knife scrape off all superfluous balsam from around the cover-glass and wipe it carefully with a cloth moistened with alcohol or benzin (or xylene). Seal as with glycerin-jelly mounts.

\section{LABELING MICROSCOPIC SLIDES.}

$\S 165$. Every permanent microscopic preparation should be carefully and neatly labeled in ink, the label being placed upon the right hand end of the slide. The label should furnish at least the following information:

\section{EXAMPLE.}

(1) The number of the preparation, the thickness of the cover-glass and of the section.

(2) The name, kind, and source of the preparation.

(3) The fixer and the stain.

(4) The date of the specimen.
No.

C. 15 .

S. $10 \mu$.

Ileum of Cat.

Transection.

Z.*H. \& E.

November, 1898.

In the case of specimens with which it is advantageous to have more information at hand a second label may be placed upon the other end of the slide, and it may bear the following information:

*It is convenient to adopt a standard system of abbreviations, thus:- $Z$. = Zenker's fluid; He. = Helly's fluid; M. = mercuric chlorid, etc.; H. = Hematoxylin; $\mathrm{E} .=$ eosin, etc. 
(1) Mode of fixation' (detail).

(2) Imbedding method.

(3) Stains employed (detail).

(4) Mounting medium (generally not necessary).

(5) Special purpose of the preparation.

A catalog giving the full data of the specimen,- -age, condition of the animal, mode of preparation in detail, special points illustrated, etc., is valuable particularly in special investigations and with standard specimens.

Paper labels are very convenient but possess the disadvantage that they are very apt to come off. In this laboratory it is the custom to label permanent preparations by means of a writing diamond with the record number at least.

$\S 166$. A carbon ink label written upon the slide itself is fairly durable and may be made as follows: First coat the end of the slide with a thin solution of balsam (xylene or benzene), and permit this to dry thoroughly. Upon this surface it is possible to write neatly with carbon ink and after the ink is dry a second coating of the thin balsam or of shellac may be given. This method is recommended.

\section{SLIDES AND COVERS.}

The slides and cover-glasses used in histological work are often slightly greasy and should be cleaned before using. After they are cleaned they should be handled by the edges only, or with forceps.

$\S 167$. Cleaning Slides. For ordinary work it is enough to wipe the slides out of clean water to which about $5 \%$ ammonia has been added. A clean glass towel free from. lint should be used and after the slides are cleaned they should be stored in covered glass jars away from the dust.

$\S 168$. Cleaning Cover-glasses. Place them in $95 \%$ alcohol to which $1 \%$ of hydrochloric acid has been added. A clean, soft cloth such as an old linen handkerchief, gauze, etc., should be used for wiping them. In wiping a cover-glass "grasp it by the edge with the left thumb and index. Cover the right thumb and index with the cleaning cloth; grasp the cover between the thumb and index and rub, the surfaces keeping the thumb and index well opposed on directly opposite faces of the cover so that no strain will come upon it, otherwise the cover is liable to be broken." (Gage, 17).

Cover-glasses when cleaned should be stored in covered glass boxes, or in Petrie dishes.

$\S 169$. Cleaning Mixture for Glass. For special purposes, such as when the slide or cover-glass is to be used in the preparation of blood smears, the cleaning 
mixture whose formula is given below may be used. Place the cover-glasses in this mixture, one by one, and permit them to remain over night or longer. Rinse them thoroughly in running water until all color of the dichromate has disappeared rinse them again in distilled water and transfer to $95 \%$ alcohol, out of which they may be wiped.

$\S 170$. Dichromate Cleaning Mixture. Formula:-Potassium dichromate, 200 grams; water, 800 c. c.; strong sulphuric acid, 1200 c. c. Dissolve the dichromate in the water by the aid of heat, and to the solution add slowly the sulphuric acid. The two fluids should be mixed in a lead-lined kettle [17].

$\S 171$. Used slides and cover-glasses, vials and other glassware that have been used with balsam, cedarwood oil, or other oily substance, etc., may be cleaned by boiling them with a solution of strong soap, such as "gold dust," one or more changes. Used xylene or toluene is sometimes useful. Slides and covers may require a second cleaning with the cleaning mixture. If only water, glycerin or glycerin-jelly has been used on them, they may be cleaned with water, preferably warm water, and then, if necessary, wiped out of $50 \%$ alcohol.

$\S 172$. Measuring the thickness of the cover-glasses. With the coverglass measurer determine the thickness of the cover-glasses and sort them into three groups: (a) those with a thickness of .13-.17 mm., (b) those less than $.13 \mathrm{~mm}$., and $(c)$ those thicker than $.17 \mathrm{~mm}$. Groups $(a)$ and $(b)$ only should be used; (c) should be discarded or used only with objects for low magnification.

It is advantageous to know the thickness of the cover-glass on an object for the following reasons: (a) That one do not try to use objectives in studying the preparation of a shorter working distance than the thickness of the coverglass [17]; (b) In using adjustable objectives with the collar graduated for different thicknesses of cover, the collar might be set at a favorable point without loss of time; (c) For unadjustable objectives the thickness of cover may be selected corresponding to that for which the objective was corrected [17]. Furthermore if there is a variation from the standard one may remedy it in part at least by lengthening the tube if the cover is thinner and shortening it if the cover is thicker than the standard [17].

\section{THE MICROTOME KNIFE.}

Finally, the microtome knife or section razor should receive a passing word as upon it depends far more than may be at first suspected the excellence of results. Scrupulous care should be taken to maintain a keen edge, smooth and free from nicks or corrosion. Never touch the edge with anything hard or metallic. Keep it clean. Before and after using it is advisable to strop it upon a strop backed with wood, and occasionally it will be necessary to hone it.

$\S 173$. For microtome knives two grades of hones are serviceable; the yellow Belgian for first sharpening and either a blue-green water hone or a fine Arkansas oil stone for finishing. These should be kept clean and free from dust. If a section knife is used a great 
deal, it is best to put it on the fine hone for a short time before each day's sectioning. Unless experienced in sharpening section knives, it is well for the first few times to work under the direction of one skilled in the manipulation. There are, however, excellent accounts to be found in works on technique [e. g., 17, 21a].

Do not be satisfied with any but a smooth edge, keen enough to cut without "pulling," a hair held in the fingers, a quarter of an inch or more from the fingers. When once a good edge is secured, take pains to preserve it.

With the yellow hone, use a lather of olive oil soap; the bluegreen soap stone is rubbed up with water; with the Arkansas stone apply a good thin oil.

\section{SPECIAL METHODS.}

\section{THE CELL.}

The technique of the Cell is almost coextensive with that of histology as a whole, at least as far as concerns the application of the more exact and delicate methods. It is necessary therefore to give here only the more salient points and accepted methods.

§ 174. General Methods. Hermann's fluid, Flemming's fluid, Zenker's fluid, Mercuric chlorid, Carnoy's fluids, and Picro-acetoformol are standard fixers, although special problems may demand other combinations. Iron Hematoxylin, the Ehrlich-Biondi-Heidenhain triple mixture, Safranin, Gentian Violet and Orange G. are perhaps the most serviceable stains. Of these iron hematoxylin is particularly and universally useful. The Ehrlich triple stain is valuable in the more analytical work and should follow Mercuric chlorid (or Zenker's fluid, Carnoy's fluid) fixation. Safranin and gentian violet, separately as red or blue stains, or successively followed by orange G (Flemming's triple stain) only after Hermann's fluid, or Flemming's fluid, or similar mixtures.

$\S 175$. Chromatin. While regressive stains of the iron hematoxylin type give valuable chromatin stains, they are not as a rule analytical or selective. In accordance with the recommendation of Heidenhain it is better to stain progressively with dilute solutions if a pure chromatin (basichromatin) stain is desired. 
Methyl green is one of the most delicate and precise of chromatin stains. It may be used alone or in combination, as in the Ehrlich-Biondi mixture ( $\$ 111)$. A very dilute hematoxylin $(\$ 88$, 92) is excellent.

\$ 176. Nucleoli. The Ehrlich-Biondi may be recommended to bring out other nuclear structures, such as nucleoli, although other combinations of basic and acid stains may be used. Montgomery recommends Ehrlich's hematoxylin followed by a strong aqueous eosin.

$\S 177$. Cytoplasm. Quite different pictures are obtained by the use of fixers as similar in composition as Flemming's fluid and Hermann's fluid, the difference seeming to be due to a varying preservation of cytoplasmic "granules." The ground work of the cell body, the so-called Spongioplasm, may be preserved by such fixers as Zenker's fluid, Flemming's fluid, Hermann's fluid, Carnoy's fluid, etc., and iron hematoxylin and the Ehrlich-Biondi mixture give satisfactory stains. The granules that may be present are of different kinds and often not easily interpreted; they include,-(a) "reserve" material (yolk granules, fat granules, etc.), (b) basophile, acidophile, neutrophile granules, (c) granules less easily preserved which include many secretion granules, etc.

(a) Yolk granules. If present in large amount a special fixer may be indicated $(\$ \S 20,21,22)$.

Fat granules; see $\S \S 224$.

(b) Basophile granulations (Granoplasma, Unna), may be demonstrated by simple alcohol fixation and subsequent differential staining with basic dyes. Compare $\S 195$ and the special technique of blood ( $\$ 211-)$.

(c) These require special technique: in general, (1) the employment of oxidizers such as dichromates, osmic acid, formalin, (2) no acid, or a minimum amount. This seems to indicate the presence of reducing substances usually lipoid in nature whose combinations are soluble in (or rendered soluble by) acid. Here belong the Mitochondria of Benda.

§ 178. Mitochondria. Benda's Method.

1. Fix in Flemming's fluid with the acid reduced to 3 drops; 2. Rinse in water, $1 \mathrm{hr} . ; 3$. Place in equal parts pyroligneous acid and $1 \%$ aqueous solution of chromic acid, 24 hours; $4.2 \%$ potassium 
dichromate, 24 hours; 5 . Running water, 24 hours; 6 . Alcohols; paraffin imbedding; 7 . Sections $5 \mu ; 4 \%$ ferric alum for 24 hours; 8. Rinse in distilled water and-9. Place in a mixture of saturated alcoholic solution of sodium sulphalizarinate 1 c. c., distilled water, 80 to 100 c. c., 24 hours; 10 . Rinse away the stain with water; 11 . Stain on the slide with a freshly prepared mixture of saturated alcoholic solution of crystal violet and aniline water, equal parts, warming until it steams; 12. Rinse in water and differentiate in $30 \%$ acetic acid (1 minute or less); 13 . Rinse in running water 5 to 10 minutes to remove the acid, drying with blotting paper; 14 . Dehydrate rapidly in absolute alcohol, (dip); 15. Clear in bergamot oil and xylene; 16. Mount in neutral balsam.

The above method is capricious. The following method is recommended: (1) fixing 12 to 24 hours in Zenker's fluid or the Copper dichromate mixture $(\$ 16)$ with $1 / 10 \%$ acetic acid only; (2) mordanting 3 to 4 days in $2.5 \%$ dichromate (or Müller's fluid or Erlicki's fluid respectively); (3) paraffin imbedding; (4) using iron or copper hematoxylin as the stain ( $\S \S 94,95)$. Benda's fluid may be substituted for the fixer, and a number of other stains may be used; consult $[6,30]$.

$\S 179$. Secretion Granules in the cytoplasm, such as the zymogen granules in the stomach and pancreas, granules of the suprarenal medulla and Islands of Langerhans, etc., may be preserved and demonstrated in the same manner and probably for the same general reasons; thus:-

1. Trypsinogen granules. Fix in $1 \%$ osmic acid which preserves and browns them; or as under 2 below.

2. Pepsinogen granules. Fix in Helly's fluid or one of similar composition. Iron hematoxylin or Weigert's copper hematoxylin, or neutral stains $(\S \S 107,128,131)$ may be used.

3. Medulla of suprarenal. Apply similar fixation methods, e. g., Helly's fluid, and regressive staining with basic stains (e. g., iron or copper hematoxylin, toluidin blue, etc.).

CONNECTIVE TISSUE

$\S 180$. White (Collagenous) Fibers. Fuchsin acid is particularly valuable. Three methods of applying it for the differential staining of connective tissue follow: 
(a) Picro-fuchsin. See $\S 119$. This may be used with or without a basic counter-stain which should precede it. If a counterstain is used remember to overstain and use the picro-fuchsin to differentiate it.

(b) Orange-fuchsin. Formula: Fuchsin acid, 2 grams; Orange G., 1 gram; Glycerin, 7 c. c.; distilled water, 100 c. c. Fix tissue in Flemming's fluid. Stain sections 30 seconds; dehydrate, clear, and mount in balsam (not alkaline). Suitable also for staining the reticular tissue (lymphatic tissue).

Somewhat more delicate than the picro-fuchsin.

(c) Mallory's connective tissue stain. See $\S 120$. While not a differential stain for collaginous fibers, it is nevertheless a valuable one, and frequently to be preferred.

$\S$ 181. Elastic Fibers (Elastin). Employ either the Weigert Resorcin-Fuchsin or the Orcein methods $(\$ \S 125,126)$, or the Verhoeff [44] method (\$127).

Both the white and elastic fibers may be stained in the same preparation, the elastic fibers being stained first.

In a mixture composed of white and elastic fibers, picro-fuchsin ( $\$ 119$ ) will stain the elastic fibers a light yellow, the white fibers being colored red.

In studying the connective tissues, it should be remembered that acetic and the mineral acids cause swelling or solution (gelatinization) of the white fibers, depending upon their strength. While this improves the cutting quality of organs rich in connective tissue, it also causes vagueness in outline of the white connective tissue fibrils. It may therefore be advisable to decrease the percentage of acetic acid in the fixer when the connective tissue is under investigation.

$\S$ 182. Reticular Tissue. Fuchsin acid, Mallory's connective tissue stain, or the orange-fuchsin acid mixture may be chosen for staining this form of connective tissue. As however the cellular elements usually mask the fiber relations, if a view of the latter is desired the cells must be removed,- by a mechanical method, such as cautiously brushing the section with a camel's hair brush, or by digestion.

$\S 183$. Digestion Method. The organ to be examined (e. g., lymphatic node, spleen, etc.), is preserved in $67 \%$ alcohol, cut into sections $2 \mathrm{~mm}$. or more in thickness, washed thoroughly in water to remove the alcohol, and digested with pancreatic solution in $1 \%$ 
sodium carbonate solution in an incubator at $38 \mathrm{C}$. until examination under the microscope shows that the cells have become disintegrated and digested. The digestion fluid should be changed every day or 2 or 3 days. A varying length of time is necessary,--sometimes a month or more. Paraffin sections may also be submitted to digestion, with or without the removal of the paraffin.

The following method is recommended for the further preparation of digested tissue. After washing thoroughly in running water to remove the digestion fluid, the tissue is carefully imbedded in celloidin $(\S 61-)$ and sectioned, the sections being 20 to $30 \mu$ thick. These sections are not fastened to the slide, but after having been brought into $95 \%$ alcohol are placed in a concentrated solution of acid fuchsin in $95 \%$ alcohol to which a drop or two (per 50 c.c.) of glacial acetic acid has been added. After several minutes, the sections are rinsed in $95 \%$ alcohol to remove the excess stain, cleared in carbol-xylene and mounted in balsam. By this method the delicate morphology is preserved and a sharp stain is secured.

The method of artificial digestion with trypsin or pepsin has other applications in the histological analysis and it possesses a distinct value.

\section{CALCIFIED STRUCTURES-BONE AND TEETH.}

\section{(A). Decalcification.}

$\S 184$. For the purpose of investigating the soft structures of tissues containing lime salts, such as bone, teeth, and calcified cartilage, it is necessary to remove the lime salts before sections can be prepared in the usual way by a process known as decalcification. Solutions of a large number of acids, combined or uncombined with other substances, may be used as decalcifiers. Very satisfactory are: (1) Hydrochloric acid, 1 c. c., $67 \%$ alcohol, 100 c. c.

(2) nitric acid, 3 c. с.; $70 \%(67 \%)$ alcohol, 97 c. c., and

(3) nitric acid, 5 c. c.; saturated aqueous solution of (potash) alum, 50 c. c.; water, 50 c. c.

In the first and second formulas the alcohol, in the third the alum acts as a restrainer of the acid. The first or second of these formulas is, perhaps, better for bone; the second has a more rapid action and is possibly a better decalcifier for teeth. It is better to let the decalcification proceed slowly for a longer time in an abundance of fluid changed often, in order that the carbon dioxid may not be 
formed too fast, accumulate in the tissues, inflate and distort them. Many fixers contain acid (e. g., see $\S \S 12,14,17$, etc.), and in their action give decalcification enough in the case of small calcified objects.

$\S 185$. Directions for use. [10.] The tissue to be decalcified had best be first thoroughly fixed and hardened by one of the approved methods, and should be in $82 \%$ alcohol. In fixing, structures not needed should be removed,--muscles trimmed away from the bone, etc. Bones or teeth should be opened with nippers or a saw, so that the fluid may reach the marrow or pulp cavity.

Place the hardened tissue in the decalcifier, where it should remain until the lime salts have been entirely removed, as may be ascertained by inserting a fine needle; if any calcified matter remains there will be a gritty feeling on using the needle. The time necessary for complete decalcification will depend upon the size and density of the calcified tissue, and will vary from 3 to 15 days or longer. The decalcifier should be changed after the first day, and if the tissue is large it is best to change it subsequently two, three or more times at intervals of several days.

When decalcification is complete rinse the tissue well in water or $67 \%$ alcohol for a few minutes and place it in $67 \%$ alcohol for one or two days and then in $82 \%$ alcohol for several days, or until ready to imbed. The $82 \%$ alcohol should be changed once or twice in order that the nitric acid may be well washed out. Although paraffin in many cases may be employed for imbedding, the celloidin method is generally more satisfactory.

Hematoxylin with eosin, hematoxylin with picro-fuchsin, and hematoxylin with picro-carmine afford good stains; by staining thoroughly with hematoxylin a differential staining of bone and cartilage may be obtained. Mallory's connective tissue stain frequently gives interesting pictures.

\section{(B). Sections of Dry Bone or Tooth.}

$\S 186$. Though the general structure of bone and tooth is shown moderately well when the tissue has been decalcified ( $\$ 184)$. the Haversian canals, canaliculi and lacunae of bone and the dentinal tubules of the teeth are shown much better in sections of dried, non-decalcified, tissue rendered sufficiently thin for microscopic examination by grinding or filing. 
$\S 187$. Directions for procedure. Prepare thin transverse sections of dried bone in accordance with the directions below. Longitudinal (radial) sections and tangential (surface) sections may also be prepared in the same manner, the former to show the Haversian canals and their anastomoses, the latter to indicate the shape of the lacunae as seen in a different plane.

1. Sawing the section. Make an exact transection of a part of the shaft of a long bone. The section should be about $1 \mathrm{~cm}$. long and include the thickness of the shaft from the surface to the medullary cavity. Make the sections about $1 \mathrm{~mm}$. thick.

2. Grinding the sections. Place the piece of bone on a cork or piece of soft wood and wet it with water. File it on one side until smooth and then turn it over. Continue the filing till the piece is from .05 to $.10 \mathrm{~mm}$. thick, using the cover-glass measurer to determine the thickness. In the beginning one can press quite hard in filing; as the section thins, more care should be exercised and the pressure should lessen.

A grinder, such as a fine carborundum wheel or emory wheel connected with a variable speed electric motor is very useful and greatly expedites the preparation of the sections. The carborundum wheel should be horizontal and the sections ground on the flat surface of the wheel, water being used to carry away the bone dust.

3. Washing and drying the section. When the section is thin enough, rinse it and dry it with lens paper.

4. Mounting the sections in hard balsam. To prepare the balsam, put two or three large drops on the middle of a slide and heat the slide in some way to drive off the volatile constituents. Do not heat the balsam hot enough to produce bubbles. When the balsam chips after cooling, it is ready for use.

In mounting, have the section and a clean cover so placed that they may be easily and quickly grasped. A cork somewhat smaller than the cover-glass should be within reach, and also a stone or piece of glass upon which to quickly cool the specimen as soon as it is mounted.

Heat the slide until the balsam is well melted. Put the slide upon a piece of paper, grasp the piece of bone with the forceps and plunge it into the melted balsam; put on the cover as quickly as possible and press it down with the cork; finally put the slide on the stone or glass to cool the balsam quickly. All of this should be 
done as rapidly as possible, and if done rapidly, the air will be retained in the lacunae and canaliculi, and cause them to stand out as black spots and lines. If soft balsam were used it would soon drive out the air, and being of nearly the refractive index of bone, it would obliterate the lacunae and canaliculi. Further, if the hot balsam were not cooled quickly, the air would be driven out and balsam would take its place in the spaces.

\section{MUSCLE.}

$\S 188$. Fresh. Much of the investigational work on muscle has been done on fresh muscle, or frozen sections. For examination fresh it is advantageous to have very thin muscles. Of the several muscles that have been recommended, one of the most available is the M. cutaneus pectoris of the frog. This may be prepared by cutting the skin in the midventral line, cutting at right angles to the first cut across to the angle of the jaw, thence caudally parallel to the first cut. The skin flap so formed contains the insertion of the muscle which may now be easily dissected free and removed together with some of the tissue at insertion and origin to handle it by. It may be used for examination fresh, with the polarization microscope, etc.

$\S 189$. Isolation Methods. Nitric acid $(\S 44)$ may be used for plain muscle and for skeletal muscle. Potassium hydroxid ( $\$ 45$ ) is suitable for heart muscle.

(a) Nitric acid. Place in the nitric acid dissociator the fresh striated muscle, gland or organ containing the muscle,-(plain or striated,)-that it is desired to isolate. If it is the intention to work out the anatomy of the muscle or the relation of the muscular coats in an organ, the entire muscle or organ should be taken; otherwise, portions will suffice. At the ordinary temperature of the laboratory the dissociating action will have been sufficient in from 1 to 3 days; test at intervals with needles to ascertain whether the fascicles and fibers can be easily separated; or fragments may be shaken in a test tube or vial with water in order to separate the fibers.

When the dissociation is sufficient pour off the acid and wash the muscle gently but thoroughly with water. If the tissue is to be stained with hematoxylin or carmine, or kept for any length of time, drain off the water and add a half-saturated solution of alum. For 
permanent storage, pour off the alum solution and place successively in $67 \%$ and $82 \%$ alcohol.

For temporary examination, tease out a portion of the muscle in water, separating the fibers carefully by means of needles; cover and examine.

Permanent preparations. (a) unstained. After teasing out with the needles drain off the water and add a small drop of glycerin or glycerin-jelly; cover, and seal after first properly cleaning $(\S \S 162$, 163). (b) stained. - Either employ the nitric acid method given above or (better; Badertscher) dissociate at incubator temperature (about $38^{\circ} \mathrm{C}$.) in a sat. solution of mercuric chlroid with $10 \%$ of strong nitric acid added. Test at intervals and when dissociation has proceeded far enough, wash out the dissociator with water and $67 \%$ alcohol. Stain with carmine or hematoxylin. Mount in glycerin ( $\$ 154-)$, glycerin-jelly $(\$ 155)$, or dehydrate, clear and place in dilute balsam (§ 157-Cf. § 215).

(b) Potassium hydroxid. Place in the fluid small pieces of the heart muscle of a fetal, new-born or young animal; after 10 or 15 minutes, the tissue should be tested with needles at intervals of about five minutes, so that the action may not be too prolonged; probably 15 to 30 minutes will suffice. As soon as the elements separate readily, pour off the caustic potash solution and add an abundance of $60 \%$ solution of potassium acetate (potassium acetate, 60 grams; distilled water, 40 c. c.). Take small fragments and tease them in this solution, or shake them in a vial, until the 'cells' are separated from each other.

For temporary examination, cover, in a drop of the potassium acetate solution. For permanent preparations, drain off the potassium acetate solution and add a small drop of glycerin or glycerinjelly.

Stained preparations. Pour off the potassium acetate solution and add a half saturated solution of alum, letting it remain for $\mathbf{2 4}$ hours or longer. Tease in water, stain with hematoxylin or carmine, wash away the stain with water, and add a drop of glycerin or glycerin-jelly. Cover and seal ( $\$ 162-)$.

If a large amount is desired, the tissue may be carried through the various steps in a vial.

$\S 190$. Sections. To bring out the structure of the fibrillae picro-aceto-formol $(\S 17)$ or an alcoholic fixer $(\$ \S 23,24)$ is preferable 
although the sarcoplasm is not so well preserved. The muscle should be moderately distended upon cork, before fixing, the ends secured by small pins: $10 \%$ formalin or Zenker's fluid or Mercuric chlorid may also be used, the sarcoplasm being much better fixed in dichromate, osmic acid or formalin mixtures. Iron hematoxylin is particularly indicated as a stain for muscle ( $\$ 94)$. Mallory's connective tissue stain $(\$ 120)$ is found to give an excellent differentiation (Kingery).

To differentiate muscle in situ, picrofuchsin may be used, which stains muscle yellow or orange, the surrounding connective tissue red. Mallory's connective tissue stain is also frequently useful.

To differentiate the intercalated discs of cardiac muscle, fix in mercuro-nitric mixture $(\$ 20)$; imbed in paraffin; stain sections with haemalum ( $\$ 89)$, diluted, 12 to 24 hours; differentiate with acid alcohol; dehydrate, clear, mount in balsam.

\section{THE NERVOUS SYSTEM.}

An analytical grouping of the numerous methods used in the study of the finer structure of the Central Nervous System is premature. The most salient point is the prominent part that reduction processes seem to play. The more important methods here presented deal (a) with the finer structure of cell and fiber;- demonstration of the tigroid substance and fibrillae; (b) the differential staining of the myelinic nerve fiber (Weigert and Marchi methods); (c) the morphology of the elements (neurones) as revealed by the chromesilver impregnation methods (Golgi methods) or the use of intravitam (methylene blue) methods.

$\S 191$. Isolation of Nerve Cells. Employ formaldehyde dissociator for the isolation of the nerve cells of the spinal cord and of the cerebral cortex, proceeding as follows:

Split the spinal cord along its median plane, separating thus the two halves, and place it in an abundance of the dissociating fluid. The cerebral cortex should be cut into small pieces by sections vertical to the surface. Allow it to remain in the dissociator from 2 to 24 hours; for the best results a stay in the flujd of more than 24 hours is not so satisfactory; although isolated cells are readily obtained their processes are broken off much nearer the cell body. 
Place a fragment of the gray matter of the spinal cord or the cortex of the cerebrum on a clean slide in a drop of $1 / 4 \%$ Congo Red ( $\$ 113$ ) or $1 / 10 \%$ eosin in formaldehyde dissociator; with the blade of a scalpel crush the tissue, grinding it thoroughly with a rotary movement, which will reduce it to small pieces. Gather the debris, drain off the fluid, and add a drop of glycerin containing stain. Cover and examine, tapping the cover sharply with the handle of the scalpel to shake out the processes of the cells and free them from surrounding matter. Examine, searching for cells with many and long processes, and if a satisfactory preparation, seal according to $\S 119$.

$\S$ 192. Isolation of Nerve Fibers. (a) For isolation of myelinic nerve fibers, with the preservation and blackening of the myelin, and for amyelinic fibers, employ osmic acid dissociator ( $\$ 42)$.

(b) For the isolation of myelinic nerve fibers with the removal of the myelin for the demonstration of the axis cylinder, neurolemma and framework of the sheath, fix nerves in Dichromateacetic or similar fluid one or more days, wash in water, pass up through the alcohols, dehydrate, remove the myelin by placing the tissue in a fat solvent,- chloroform, for one or more days, $95 \%$ alcohol 1 day, pass through the alcohols to water, stain in Delafield's hematoxylin 12 to 24 hours, wash in water, pass up through the alcohols, dehydrate and place in clearer. Out of this the small bundles of the nerve fibers may be teased apart with needles, care being taken to keep the fibers as nearly parallel as possible. Mount in balsam.

$\S 193$. Gold Chlorid Methods. These methods, which are widely serviceable, depend upon the reduction of gold chlorid solutions by the tissues through the agency of (a) sunlight or (b) various chemical substances of which the acids, formic, acetic, citric, etc., are particularly used. Either one or both of these agencies may be used. Usually fresh tissue is used although the method may be applied to fixed tissue, particularly as a neurofibrilla stain [6, 30]. It is the method par excellence for staining motor nerve terminations for which purpose the following method is serviceable.

$\S 194$. [21]. Fresh tissue or (better) tissue fixed in $10 \%$ formalin may be used. Place small pieces of muscle containing the endings for 30 minutes in $10 \%$ formic acid solution. Remove to $1 \%$ gold chlorid for 30 to 40 minutes, avoiding direct sunlight; the tissue becomes yellow. Transfer again to a $2 \%$ formic acid solution in which the tissue should remain for 1 or 2 days in the dark (rich purple 
color). A bluish-purple indicates too great a reduction. Wash in distilled water for an hour or so.

If it is desired to make a teased preparation, transfer to glycerin in which the fibers may be cautiously teased apart, taking care not to separate them too much. Permanent mounts may be made in glycerin or glycerin-jelly.

If sections are called for, dehydrate, clear (xylene method, $\$ 52$ ), and imbed in paraffin. Celloidin may also be used.

With fresh tissue the treatment by formic acid may cause too marked a swelling and distortion; in which case the Ranvier method [30] may preferably be used.

$\S 195$. Tigroid substance (Nissl's bodies). The stainable substance in the cell body of nerve cells resembles the chromatin of the nucleus in its reactions, staining with basic stains of which a number are suitable. Alcohol, Carnoy's fluid ( $\$ 26,27)$, mercuric chlorid, or formalin may be used as fixers, preferably one of the first three. Methylene blue or toluidin blue are the usual stains.

$\S 196$. Nissl's Method (modified). Imbed in paraffin or celloidin tissue that has been fixed in $95 \%$ alcohol or Carnoy's fluid; cut the sections rather thick, 15 to $20 \mu$. The sections may either be fastened to the slide or carried on as free sections. Stain the sections in a $1 \%$ aqueous solution of methylene blue or toluidin blue for 5 to 10 minutes, heating it until it steams. Permit it to cool, rinse in water, dehydrate and differentiate in absolute alcohol, clear with oil of cajuput and xylene and mount in xylene balsam. The nerve cells and nuclei will be stained blue, all else colorless. In the cell-bodies of the nerve cells, the tigroid substance will be stained. Should the stain not be selective enough, differentiate for a few seconds before dehydrating with a mixture of anilin 1 part, $95 \%$ alcohol 9 parts.

If celloidin is used for imbedding, it should be remembered that it should be dissolved away in the differentiation (absolute alcohol).

$\S$ 197. Held's Method (modified). Fix in mercuric chlorid (or as for Nissl's method), imbed in paraffin, cut sections 5 to $10 \mu$ and stain 15 minutes in $1 \%$ solution of erythrosin in $67 \%$ alcohol, rinse, stain 10 minutes in $1 \%$ aqueous solution of toluidin blue, differentiate briefly in $1 / 10$ th $\%$ alum solution, dehydrate rapidly in 95\% and absolute alcohol, clear in xylene, mount in balsam. Alkaline methylene blue or Nissl's soap solution $(1 / 2 \%$ methylene blue in $1 / 5 \%$ soap solution) may be used in either of these two methods if desired. 
$\S 198$. Neurofibrillae (Simarro-Cajal Methods). Three of Cajal's methods may be given: Formula $3 a$. 1. Fixation in ammoniacal alcohol ( 2 to 10 , usually 4 to 5 , drops of ammonia per 50 c. c. of 95\% alcohol), 20 to 48 hours; 2. Mop up with absorbent paper and 3. Place in $1 \frac{1}{2} \%$ silver nitrate solution for 4 to 5 days at 32 to $40^{\circ} \mathrm{C}$.; the tissue when ripe should be light gray; 4 . Wash for a few minutes in distilled water; 5 . Reduce in a solution of 1 to 2 grams hydrochinon or pyrogallol; water, 100 c. c.; formalin, 5 c. c.; for 24 hours; 6. Wash in water; 7. Imbed by the paraffin method; 8. Section and mount in balsam or damar. Recommended for spinal cord, cerebellum, spinal ganglia.

Formula 4a. 1. Fix small pieces of tissue ( $5 \mathrm{~mm}$. thick or less) for 6 to 12 hours in formalin, 15 c. c., water 85 c.c.; 2. Wash 6 hours or longer in running water; 3. Place for 24 hours in 50 c. c. of alcohol with 5 drops of ammonia added; 4 . Mop with absorbent paper; 5 . Place in $11 / 2 \%$ silver nitrate solution for 4 to 5 days (35 to $38^{\circ}$ C.); The remaining steps as in formula 3a. Recommended for sympathetic ganglia, cerebrum, cerebellum.

Formula 5a. 1. Fix small pieces for 6 to 8 hours in water and pyridin, each equal parts; then for 18 to 24 hours in pure pyridin; 2. Wash for several hours in running water; 3. Place in $90 \%$ alcohol for 1 day; 4. Mop with absorbent paper; 5. Place in $1 \frac{1}{2} \%$ silver nitrate solution for 4 to 5 days $\left(35\right.$ to $38^{\circ} \mathrm{C}$.); The remaining steps as in 3a and 4a. Recommended for embryonic and fetal tissue (neurogenesis), regeneration, cerebrum.

By these "photographic" methods, fibrillae, fibrillar networks, changes in histogenesis, etc., may be demonstrated.

For other methods of demonstrating the neurofibrillae,-Bielschowsky's, Bethe's toluidin blue method, Apathy's hematein method, etc., consult larger works on technique $[6,30]$.

$\S 199$. The Weigert Method for staining differentially the myelin of myelinic nerve fibers. This method in all its various forms, depends upon the power of the myelin, probably through the reducing fatty acid present, to combine with and hold in (nearly) insoluble form the chromium (oxid), which thus serves as a primary mordant for a copper or iron hematoxylin stain, which is subsequently differentiated by an oxidizer as a bleacher. The important steps are:-(1) fixing and mordanting in dichromate solutions; usually the potassium salt is chosen; (2) a second mordantage in copper (acetate), (3) the staining; (4) the differentiation. The point at which the imbedding and sectioning are introduced is of secondary 
importance. It should be remembered, however, that the fatty substances (lipoids) of the myelin upon which the method depends are soluble in the reagents of both the paraffin and celloidin methods, less so in the latter-and in acids, and that even the dichromate mordantage does not preserve them perfectly. The dichromate mordantage must thus be given before the imbedding is begun (alcohols), and preferably with the fresh tissue. Practically the only fixer that is indifferent in this respect and after which the dichromate may first be used is formalin. Other dichromate fixers such as Zenker's fluid with the acetic acid reduced to about $1 / 5 \%$ may be used, but it is well to let them act only a relatively short time, and continue the mordantage with simple dichromate solutions. Aside from this the point at which the imbedding and sectioning are introduced is of secondary importance; thus, Strong [41] combines the copper and chromium mordantage by using copper dichromate; the former may also follow the dichromate treatment before imbedding is begun, in case of celloidin imbedding it may be applied to the celloidin imbedded block, or after the sections are cut. Streeter [40] stains (in toto, ) 4 to 6 days as well as mordants before the imbedding. Weigert has added to both the primary and secondary mordants chromium fluorid to (1) hasten the process and (2) prevent precipitates; the formulas are: (a) $5 \%$ potassium dichromate, 100 c. c.; chromium fluorid, 2 grams; (b) $5 \%$ copper acetate, 100 c. c.; chromium fluorid, 2 grams; glacial acetic acid, 4 c. c. (b) is especially indicated if the secondary mordantage is given before the sectioning. Whatever modification of the method is employed, the reduction of the dichromate by the tissue in the primary mordantage should fully reach the dark brown stage, but not pass it (i. e., become green). Sheldon [4] gives a good resume of the method. The following method is serviceable:-

1. Fix tissue for 1 to 2 days in Zenker's (see above) Orth's, or Helly's fluid, $10 \%$ formalin, Müller's fluid, or potassium dichromate solution.-

2. Mordant until dark brown in $3 \%$ and $5 \%$ aqueous solution of potassium dichromate. This usually takes about 4 weeks; or, shorten the period by using the dichromate-chromium fluorid mixture (above) when about 5 days should suffice;

3. Wash in running water 1 or 2 days, and

4. Pass up through the alcohols, preferably keeping the tissue in the dark. 
5. Imbed in celloidin or paraffin.

6. Stain and differentiate sections by the Weigert copper hematoxylin method ( $\$ 95)$.

7. Mount in neutral or alkaline balsam.

Large sections are usually best carried on as free sections. The differentiation of the stain should be carefully watched and stopped when the fibers are a rich dark blue on a yellow-brown-background. The reagents used in dehydrating, clearing and mounting should be neutral or alkaline, - not acid.

$\S 200$. Pal's method may be used if it is desired to stain the nerve cells subsequently.

Fix and mordant, in the dichromate as above; omit the copper mordantage; imbed and section, staining the sections with the strong hematoxylin used in the Weigert method ( $\$ 199)$ until the sections are a blue-black.

Rinse the sections in tap water and differentiate by treating for a short time (20 to 30 seconds) with a $1 / 10 \%$ aqueous solution of potassium permanganate and for a few seconds with a mixture of $1 \%$ oxalic acid and $1 \%$ potassium sulphite, equal parts. The action will be very rapid and must be carefully watched. Wash the sections $1 / 2$ hour in running water. Counter-stain with a red stain (eosin, erythrosin, carmine, etc.) if desired.

$\S$ 201. Marchi Method. This method of staining differentially degenerating myelinic nerve fibers depends upon the fact that potassium dichromate (or chromic acid) is able to satisfy the reducing power of myelin but does not oxidize the globules of free fatty acid (?) formed in the degeneration of the myelinic sheath of the fiber, which may be subsequently blackened by the reduction of osmium tetroxid. Important points in the successful application of the method are: (a) the length of time the degeneration should be allowed to proceed before treating with potassium dichromate, (b) the time in the dichromate mordant, (c) the time in the osmic acid mixture (sufficient and complete penetration), (d) the preservation in situ and final mounting of the osmicated fat granules; the difficulties here are those of fat preservation in general ( $\$ 224)$.

(a) The optimum will vary and must often be determined experimentally: in general,--for cold blooded animals; (Toad), 30 to 40 days; for mammals, 12 days.

(b) 8 to 10 days in Müller's fluid or $3 \%$ potassium dichromate is usually enough; a longer time does no harm. 
(c) The osmic acid is useful in $1 \%$ aqueous solution usually mixed with a potassium dichromate solution. 6 to 10 days suffice; a longer time does no harm (brittleness).

(d) See below.

\section{§ 202. As employed by van Gehuchten [18]:-}

1. Harden in $3 \%$ potassium dichromate solution for 3 weeks;

2. Transfer to a mixture of $1 \%$ osmic acid solution, 1 part; $3 \%$ potassium dichromate, 4 parts; for 3 weeks, blocks of tissue not more than $2 \mathrm{~mm}$. thick. Use abundance of the fluid and change 2 to 3 times if deemed necessary.

3. Wash in running water for 12 to 24 hours.

Avoid the paraffin and celloidin imbedding methods if possible. Of these two the celloidin method is preferable. For further treatment, see $\S 227$.

$\S$ 203. Flemming's or Benda's fluids $(\S \S 19,227)$ may be used instead of the Marchi method for the same purpose with small objects, and peripheral nerves, etc.

$\S 204$. The Golgi Methods. whose field of application is not confined to the nervous system (gland ducts, bile capillaries, blood capillaries, secretory canaliculi, muscle, etc.) consist in (a) mordanting the fresh or living tissue for a sufficient length of time in a dichromate solution, usually containing as well osmic acid or formalin, and then-(b) transferring to a silver nitrate solution, whereupon certain of the nervous elements become outlined more or less completely by impregnation with a chrome-silver combination.

The reaction probably depends on the presence, in certain "physiological states" of the elements of a substance or substances which combines with the chromium salt (with reduction?) and through it with the silver salt. These hypothetical substances,--or possibly physical states,-seem to disappear more or less rapidly after the death of the animal and their power to hold the silver in combination to decrease with the progress of the dichromate mordantage beyond a certain point. If successful, certain of the cells and their processes, -amyelinic, and to a certain extent, myelinic nerve fibers, are outlined by an impregnation, black by transmitted, brown by reflected light.

$\S 205$. The method is, however, capricious; success depends on (a) the kind of animal; different parts and tissues react more satisfactorily in some animals or classes of animals than in others. 
The age of the animal; some regions of the nervous system give better results in young or fetal animals; 'other parts take the stain better in older animals, etc. (c) The time of mordantage; it is necessary that the tissue be mordanted a certain length of time, constant (relatively) for a certain kind of tissue under the conditions above $(a$ and $b$ ). It is necessary that the best amount of dichromate mordantage be given. (d) Different organs and regions of the central nervous system vary greatly in the ease with which they can be made to furnish satisfactory impregnations. Almost certain impregnations of hippocamp can be gained; cerebral cortex is likewise quite easy to stain. With the olfactory bulb the action is not constant though fairly complete. The optic lobes' and retina of birds and large reptiles are more satisfactory than those of mammals. The myel (spinal cord) of embryo birds ( 7 to 14 day chick best) is generally more satisfactory than that of mammals; in any case, fetal or new-born animals should be employed. Difficult are satisfactory impregnations of sympathetic ganglia, organs of special sense and the intrinsic nerves of the viscera.

The important forms of the method are:-(a) the Slow Method; mordantage in dichromate solutions of preferably increasing strength, $2,3,5 \%$, for 1 to 4 months depending on the temperature, strength of solution, etc. (b) The Rapid Method; in this another oxidizer, osmic acid, is combined with the dichromate with a reduction in the duration of the treatment to a few days. Combination of (a) and (b) are sometimes serviceable; (c) Double (or triple) Impregnations, obtained by repeating (b). Important modifications are: (1) substitution of formalin for the osmic acid in (b), (2) mercuric chlorid instead of silver nitrate (Cox's Method). The Golgi methods have been widely applied and for details the individual papers may be consulted [6].

$\S 206$. Golgi's Rapid Method. This is the most generally serviceable of the different methods.

Directions for use. Tissue of a (preferably) young animal is placed in a mixture of 4 parts of $3 \%$ potassium dichromate and 1 part of $1 \%$ osmic acid. The amount of the fluid should be at least twenty times the bulk of the tissue and should be changed as soon as it grows turbid or loses the strong characteristic odor of the osmic acid.

After the action has proceeded to the right degree ( $\$ 207)$, rinse the tissue in water for about 5 minutes and place for 15 minutes in 
a $1 / 4 \%$ solution of silver nitrate, and then for 2 or more days in a $3 / 4 \%$ solution of silver nitrate, preferably keeping it in the dark.

Without washing, imbed rapidly in celloidin as follows:

(a) Dehydrate 2 to 3 hours in $95 \%$ alcohol, changed two or three times; (b) place in thin celloidin for $\mathbf{2 0}$ minutes, in thick celloidin for 20 to 30 minutes; (c) imbed in thick celloidin, on a block of wood (best ; $\S 65 a)$; $(d)$ harden the mass in chloroform for 20 to 30 minutes, and (e) place the block in clarifier and cut, sections being 50 to $100 \mu$ thick according to the nature of the tissue and the character of the impregnation.

(f) Place the sections in $\mathbf{9 5} \%$ alcohol for a few minutes; clear in carbol-xylene and mount in balsam by placing the section on the slide, absorbing the clearer thoroughly by means of tissue paper and spreading over it thick xylene balsam. Do not cover. Later, when the balsam has hardened somewhat, it may be melted by heat and much of the superfluous balsam drained from the section and scraped away with a knife, and a cover glass added if desired.

$\S 207$. Time of hardening. From results of Cajal, van Gehuchten, and others, and from general laboratory experience, the following periods will probably be found approximately correct. In general: The best results are to be obtained with kittens 3 to 20 days old, puppies 2 weeks old, rats 8 to 10 days, rabbits 8 days. (a) For cerebral cortex (and hippocamp): New-born kitten, 1 to 2 days; kitten half grown (3 to 4 months), 3 to 4 days; new-born rabbit, 24 hours; rabbit one month old, 2 to 3 days; adult mice, 3 to 4 days.

(b) For spinal cord: Chick of 5 to 6 days' incubation, 24 hours; chick, 14 to 15 days' incubation, 3 days; new-born kitten or puppy, 2 to 3 days. Frog tadpoles (large) 3 to 5 days.

(c) Cerebellum: New-born kitten, 1 to 2 days; kitten half grown, 4 days.

(d) Sympathetic system: Chick of 14 to 18 dars' incubation, 3 days.

(e) Retina: 1 to 3 days.

(f) Olfactory mucous membrane: 3-4-7 days.

$\S$ 208. Intra vitam Methylene Blue. Methylene blue shares with a number of coal tar dyes the power of staining during life nerve cells and fibers and certain cytoplasmic granules. Like the Golgi methods, "intra vitam" staining of nervous tissue is capricious; applicable only to living or fresh tissue and depends upon unknown 
substances or conditions that become changed after death. In the reaction reduction of the methylene blue to its leucobase (colorless) by the nervous tissues appears to play an important part. In general, the technique involves: (1) Bringing a methylene blue solution of sufficient strength in contact with the (essentially) living nervous elements, (2) permitting it to remain a sufficient length of time for the staining reaction, (3) exposing the tissue to the action of the oxygen of the air until the stain is fully developed,-re-oxidation of any leuco-methylene blue and satisfying the reducing reaction of the tissue; then-(4) either examining at once or fixing the stain in situ by its precipitation in an insoluble form for its preservation (imbedding and sectioning).

1. The methylene blue may be brought in contact with the neurones by injection, (a) through the vascular system,--aorta if the animal is small, artery supplying the part, if large;-(b) into the body cavities; (c) subcutaneously: or, by immersion of the organ or part, or the entire animal if small (many invertebrates). Keeping in mind the end result desired, the best method will suggest itself in a particular case. Cajal cut parallel slits in the cerebral cortex and inserted the methylene blue in powdered form or as a saturated solution. In general, the solutions should be as dilute as possible; of $1 / 15$ to $1 / 4 \%$ strength in physiological salt solution, the more direct the application the weaker. It is well to have on hand a $1 \%$ stock solution in physiological salt solution and dilute it (with salt solution) as desired. Of the methylene blue preparations that are available, Ehrlich's or "B. X." are more generally used. A combination of injection and immersion is often advisable. In introducing the stain by injection, first remove the blood by washing out with physiological salt solution or by bleeding and let the injection be full, i. e., through the capillaries into the veins. If a mammal is being dealt with, salt solution and staining solution should be warmed to body temperature ( 35 to $38^{\circ} \mathrm{C}$.).

2. It is difficult to give any general rules as to the time the methylene blue solution should remain in contact with the tissue before exposure as it is best to determine it experimentally in each case. The time should be shorter for warm blooded, longer for cold blooded animals. If introduced by injection, 20 to 30 minutes for a mammal, 2 to 12 hours for a cold blooded form, may be suggested; the organ or part should then be removed, wet with the dilute stain (perhaps $1 / 15 \%$ strength) for another period of time, $-1 / 2$ to 1 hour, 
access of oxygen to the point desired being kept in mind. At intervals, free hand sections should be examined under the microscope to determine the state of the reaction. If the tissue is mammalian, it should be kept protected from evaporation during this time and warm, as in an incubator.

If the stain was applied by immersion, a shorter time suffices;up to 15 minutes or so with a subsequent exposure to the air of $1 / 2$ to 1 hour wet with the dilute solution.

Small aquatic animals may be immersed in very dilute solutions $(1 / 100$ to $1 / 1,000 \%)$, the optimum strength and time of immersion being experimentally determined.

In some instances (particularly parasitic worms) the tissue hold the methylene blue in reduced form in spite of exposure to the air and the color is only developed when placed in the fixer (below). In any event it is better to fix the stain earlier than later.

4. Two methods are standard for preserving tissue stained intra vitam with methylene blue, the first of these (Dogiel's) is suitable only for such tissue as may be exposed for study by teasing; the second (Bethe's) may be used both for such preparations and those which it is desired to imbed and section.

Dogiel's Method. Immerse the tissue in a saturated solution of ammonium picrate (orange-yellow needles) for 2 to 24 hours according to the size of the piece, using abundance of fluid. If maceration occurs, Dogiel suggests addition of $1 \%$ of $1 \%$ osmic acid. Transfer tissue to equal parts of the ammonium picrate solution and glycerin in which the tissue may be preserved, teased, and mounted.

Bethe's Method. Immerse the tissue in a 5 to $10 \%$ aqueous solution of ammonium molybdate for 1 to 24 hours according to the size of the piece, using abundance of the fluid. Trim the tissue as desired, removing all unnecessary parts, dividing it into smaller pieces, etc. Wash in distilled water, changed several times, for 1 to 3 hours. Dehydrate rapidly in $95 \%$ and absolute alcohol, -4 to 6 hours, shortening the time if possible. Imbed rapidly in celloidin ( $\$ 206)$ which may be hardened in $67 \%$ alcohol and sections cut.

It may be advisable, particularly in summer work, to have the water and alcohol specially cooled to prevent dissolving of the stain in the alcohol. Tissue already fixed in the ammonium picrate may be refixed in the ammonium molybdate solution. Indeed, this double fixation is recommended by Bethe as particularly suitable for invertebrate material. 
The paraffin method is not advisable if the above method suffices. If it is desired, however, after the dehydration, clear thoroughly with oil of cloves followed by xylene and infiltrate in paraffin (\$ 52). In treating the sections, avoid alcohol as much as possible. Section or in toto staining may be applied, preferably carmine (not alkaline or acid formulae).

This important method has been elaborated largely through the work of Bethe, Cajal, Dogiel, Huber, Retzius and others, for which consult [6].

The method may be used for the staining of Neurofibrillae; for its applications for this purpose, consult the special articles.

$\S 209$. Neuroglia Stain. Tissue is fixed for 24 hours in copper dichromate-sublimate-acetic (1/5\%) mixture ( $\$ 16)$ and subsequently mordanted 3 or 4 days in $2.5 \%$ copper dichromate. Imbed in paraffin, Sections ( 5 to $10 \mu$ ) are fastened to the slide and the Benda stain is used, as follows:-(1) $4 \%$ ferric alum for 24 hours; rinse well in distilled water and (2) place for 24 hours in a dilute solution (amber yellow) of sodium sulphalizarinate (concr. sol'n. in $70 \%$ alcohol added to distilled water). Rinse in distilled water, blot with absorbent paper, and stain (3) in a $1 / 10 \%$ aq. sol'n. of toluidine blue, heating it until it steams, cooling and staining 15 minutes. (4) Rinse with distilled water and treat for a few seconds with acid alcohol $(70 \%$ alcohol, 100 c. c.; concr. Hcl, 6 drops). (5) Blot with absorbent paper and dehydrate rapidly with $95 \%$ and absolute alcohol.

Differentiate carefully with creosote, to the right degree; (7) blot with absorbent paper, and rinse in several changes of xylene. Mount in balsam. Neuroglia fibers, a dark blue, neuroglia 'cells' a light blue, axis cylinder and myelinic sheath red to brown, nuclei dark blue, etc.

This method may be used with fresh or formalin material, human or animal. (Kingery).

THE BLOOD.

Special methods in the examination of the blood include (1) Examining fresh; (2) Technic of staining blood films; (3) Determination of the number of red and white corpuscles per cubic millimeter; (4) differential counting of the white corpuscles; (5) Determination of the relative amount of hemoglobin; (6) Spectroscopic examination of blood (hemoglobin), etc. (1) and (2) are briefly given here; for (6) see [17]. 
§ 210. Examining fresh. This consists in covering a drop on a slide, and immediately sealing the cover-glass to prevent evaporation, observing the following cautions: (1) The drop of blood (from the finger or the lobe of the ear) should flow freely and not be obtained by pressure. The drop should be a medium-sized one, which will spread out in an even, thin layer under the cover. The drop should be received upon a cover or slide, covered, and sealed at once with castor-oil.

Examination of fresh blood may be used in clinical examination for the detection of some abnormal conditions, and it is of value in the rough diagnosis of many others.

$\S 211$. Stained preparation of blood. (a) Preparing the blood film. This may be best done in one of two ways: (1) The edge of a slide is first drawn through a drop of fresh blood and then moved quickly across the surface of a clean cover-glass or slide, in this way spreading the blood in a thin, even layer upon the glass. Success depends upon getting the right amount of blood upon the edge of the slide and the quick, even movement by which it is spread upon the cover-glass or slide. Preparing the film on a slide is simpler and to be preferred if a differential count of the leucocytes is to be made. A second, possibly better, method is the following:

(2) Have ready two thin clear cover-glasses (or slides) and obtain a drop of fresh blood. Take one of the covers in the forceps, touch it to the drop of blood and place it upon the second coverglass eccentrically, with one edge projecting slightly. Slip the two covers apart in the plane of their surfaces and dry them quickly by waving them in the air or by passing them rapidly over the tip of a flame. The lower cover-glass will have the better film.

(b) Fixing the hemoglobin with (a) ether-alcohol or heat, or (b) at the time of staining (methyl alcohol) ( $\$ 214$ ).

$\S 212$. Fixing the film. When the blood films on the covers are dry, place them in the fluid for $1 / 2$ to 1 or several hours. Let them fix for a longer rather than a shorter time, as the quality of the stain (with triacid mixture) will be improved. After they have fixed a sufficient time remove and again dry them in the air. They may now be stained, immediately or at convenience.

$\S 213$. Staining unfixed films. Eosin-Methylene Blue stains (below). Fixed films may be stained with hematoxylin and eosin as well as with other stains. If the film is on the slide balsam and a 
cover-glass are unnecessary if it is to be examined with the oil immersion objective.

$\S 214$. Eosin-Methylene Blue. The most of the formulas are made on the principle of neutral stains, - eosinates of methylene blue dissolved in methyl alcohol, the staining solution being diluted with water during the staining $(\$ 85, \mathrm{~b})$. As polychrome methylene blue is generally used, the range of selectivity is increased by the presence of methylene azure.

(a) Nochts-Hastings Stain. ( $§ 130)$. Stain blood smears 1 minute with the undiluted stain, then dilute with distilled water until a metallic film begins to appear and the diluted stain appears reddish at the edge. Stain 5 minutes more, rinse quickly with distilled water, absorb excess with absorbent paper, dry in the air; when dry mount in balsam.

(b) Wright's stain ( $\S 129$ ) is of similar composition and the staining process is carried out in the same manner. Stain blood smears 1 minute with the undiluted stain; dilute drop by drop with an equal volume of water and stain for 3 minutes; rinse, dry, etc., as above.

(c) Jenner's stain ( $\S 128$ ) is a simpler stain, and easier to use. The simple (i. e., not polychrome) methylene blue is used, and the differentiation of the stain is secured in the washing out. Stain blood films 3 minutes or more, rinse a short time with distilled water (until the best portions of the film are pink). Absorb excess of water with absorbent paper and dry in the air. When dry, mount in balsam.

$\S 215$. It is sometimes advantageous, - as for class work and with non-mammalian blood,- to handle blood in bulk. The following method has been used with good results. Fix for 1 to 6 hours by having the blood drop into a vial of $1 \%$ osmic acid. The blood-cells are allowed to settle and the supernatant fluid removed with a pipette. By this method the blood is passed through, successively, - 2 or 3 changes of distilled water, $50 \%, 67 \%$ alcohols, paracarmine, $67 \%$, $82 \%, 95 \%$ and absolute alcohols, xylene, to thin xylene balsam ( $\$ 161)$ in which the blood is stored. By gently agitating, the corpuscles are evenly distributed and a drop of the balsam mounted contains numerous blood cells. This is an excellent method for preparing isolated epithelial and muscle cells for class use. Such material may be kept for years and is always ready to use. 
FINE INJECTION.

For the purpose of examining microscopically the finer arteries and veins and the capillaries in a tissue, and their relation to the other parts, it is necessary to fill them with some colored injection mass, or otherwise stain or color them. Numerous injection masses are in use; the following meet the general needs. For injection fluids for special purposes, consult the literature [6].

$\S$ 216. Carmine gelatin mass. Formula: Dry gelatin, 75 grams; carmine (No. 40), 10 grams; water, 90 c. c.; ammonia, 10 c. c.; acetic acid, q. s.; chloral hydrate, 10 grams.

Soak the gelatin in water until it is soft; pour off the superfluous water and melt it (in an agate or porcelain dish) over a water bath. Grind the carmine to a paste with water; add all the ammonia and water; filter, warm to $80^{\circ}$ or $90^{\circ} \mathrm{C}$., and add to the warm gelatin. Then add slowly the acetic acid diluted with an equal volume of water, while constantly stirring the mass, until the mass smells very slightly of the acid. Filter through fine flannel. If the mass is acid, the chloral hydrate may be safely added (as a preservative); if any ammonia is present it will decompose itforming chloroform, and a granular precipitate. If too much acid is added, the gelatin will not set.

$\S 217$. Berlin blue injection mass. Formula: Dry gelatin, 75 grams; saturated aqueous solution of Berlin blue, 150 c. c.; chloral hydrate, 10 grams. Prepare the gelatin in the manner given above ( $\$ 216$ ); warm the Berlin blue solution (to $80^{\circ}$ or $90^{\circ}$ C.), and add it to the hot gelatin. Heat the mixture for 10 minutes or more, stirring it occasionally, and filter it through fine flannel and add the chloral hydrate.

$\S 218$. For securing the best results in injecting the following conditions should be observed: (1) A young but nearly mature, lean animal is to be preferred. (2) Kill the animal with an anesthetic (chloroform) and leave it in the anesthetic at least half an hour before beginning the injection; do not, however, wait until rigor mortis sets in. (3) Inject only the part desired, tying all anastomosing vessels and all vessels to other parts. Inject into the artery of the part, leaving the vein open until nearly pure injection mass escapes, then tie it and continue the injection until the part feels hard and is the color of the injection mass. (4) When the injection is finished cool the part injected by means of cold water, ice, or snow. 
(5) Harden the injected tissue 1 or 2 days in $50 \%$ alcohol, 2 or 3 days in $67 \%$ and $82 \%$ alcohols. The acidity of the alcohols should be insured by adding to the $50 \%$ alcohol a few drops of acetic acid. The tissue may be stored in $82 \%$ alcohol until ready for sectioning. Formalin (10\%) may also be used as the fixer and preserving fluid. For sectioning the celloidin method is usually preferable.

$\S 219$. Silvering blood vessels. Silver nitrate may be used for coloring blood vessels, and thus differentiating them. See § 222 .

$\S 220$. Dense Masses, such as do not pass through the capillary network are useful in giving double injections, the veins and capillaries one color, arteries another. Two such may be mentioned: (a) Lampblack Gelatin Mass; (b) Ultramarine Gelatin Mass (Spalteholz.).

(a). Lampblack and gelatin mass in the proportion of about $1: 12$.

(b). A $10 \%$ gelatin mass to which is added ultramarine in proportion of $30: 100$.

In injecting, inject through the artery with one of the gelatin masses given in $\S \S 216$-, and follow it up with one of the above which will push the first mass through into the capillaries and veins.

\section{SILVER NITRATE IMPREGNATIONS.}

$\S 221$. The preparations stained by means of nitrate of silver are prepared as follows; The fresh tissue is washed for a minute or so in distilled water to remove from the surface all albuminous substance, and then transferred for 2 to 5 minutes or longer to a 1 to $1 / 2 \%$ aqueous solution of silver nitrate and exposed to direct sunlight until a light brown. When, by examination with the microscope, the stain was found to be sufficient it was again rinsed in water and placed in glycerin or alcohol. Employed in this manner with fresh tissue, silver nitrate stains the cell cement, affording thus negative images of the cells. If a membrane such as mesentery is to be silvered removed from the body, it should first be cautiously stretched, as over a ring, to avoid creases.

\$222. Silvering Vascular Epithelium. In order that the vascular epithelium of small arteries, veins, and capillaries should be well demonstrated, silver nitrate solutions of $1 / 4$ to $1 / 2 \%$ strength must be injected into the vessels. 
§ 223. Procedure. Connect a canula with the artery supplying the alimentary canal (superior mesenteric) or the brain (carotid) and inject distilled water until the water flows out of the returning vein colorless. Then immediately inject the silver solution until it runs from the vein. After a minute or two follow with distilled water or physiological salt solution. Place the intestines and mesentery in water and expose them to the light until they become slightly browned. Strips of the muscular coat of the intestines, especially of the rabbit, will show capillaries well. Veins and arteries side by side may be found in the mesentery. If the brain vessels are injected one can get admirable preparations showing nuclei as well as cell outline by staining in hematoxylin. Mount in glycerin, or, if desired, dehydrate and mount in balsam. The tissue may be kept in $50 \%$ alcohol or in $50 \%$ glycerin for several months before mounting if it is kept in the dark.

For large vessels and endocardial epithelium open the vessels or the heart and silver as directed above for mesentery. It may be necessary to make thin free-hand sections so that the preparation will be thin enough for high powers.

\section{HISTO-CHEMICAL METHODS.}

There are special chemical substances which it is often desirable to preserve and differentially stain. In most cases, the staining reactions are not specific enough to come under the category of microchemical tests, the evidence gained being circumstantial or indirect, the application of two or three different methods being sometimes necessary for confirmation. Such methods may be spoken of as histo-chemical rather than micro-chemical.

\section{A. Fats. (Lipoids).}

$\S$ 224. Free fats and lipoids are soluble in ether, chloroform, absolute alcohol, xylene, benzene, and essential oils. As these are necessary for paraffin and celloidin imbedding methods, especially the former, the satisfactory preservation of these substances presents some difficulties. The use of the freezing microtome is therefore particularly called for. See, however, $§ \S 227,228$.

The stains applicable to the demonstration of fats are (a) stains soluble in the fat solvents, e. g., Sudan III, and scarlet red; and (b) such as depend upon the reduction of salts by the fats (e. g., osmic acid and potassium dichromate) [29]. 
§ 225. Sudan III. Fix tissue in formalin or Müller's fluid. Cut free-hand sections, employ the freezing microtome method, or isolation ( $\S \S 35-$ ). Rinse sections in $82 \%$ alcohol and transfer sections to a strong solution of the stain in $82 \%$ alcohol; leave several minutes covered from evaporation; rinse with $82 \%$ alcohol and transfer to water. Mount in glycerin or glycerin jelly $(\S \S 151,155)$. Fat is stained red.

$\S 226$. Herxheimer's Stain. Preparation for staining is as above (\$225). Pass sections into $67 \%$ alcohol. Transfer to a strong solution of Scarlet Red in $67 \%$ alcohol rendered alkaline by $2 \%$ of sodium hydroxid. Stain for several minutes; rinse with $67 \%$ alcohol and transfer to water. Mount in glycerin or glycerin-jelly. Fat globules stained red. It affords a more intense stain than Sudan III.

Indophenol (saturated solution in 67 or $82 \%$ alcohol) may be used as a blue stain for fat in a similar manner.

$\S 227$. Osmic Acid. ( $\S 30,18,19)$. Osmic acid is reduced by the unsaturated fatty acids (e. g., oleic acid) which are blackened by it. The saturated fats and fatty acids (e. g., stearic, palmitic,) are not so blackened [1] but the black color subsequently appears when the tissue is placed in alcohol [41]. The fat so oxidized and impregnated with osmium (?) becomes less soluble in fat solvents ( $\$ 224)$ and may be retained in tissue imbedded in either paraffin or celloidin. There is, however, a difference in fats; adipose tissue is easily preserved, while some of the fat granules found in the organs require the special precautions mentioned below.

Fix sections of tissue 2 to $3 \mathrm{~mm}$. thick in Flemming's or Benda's fluid for 2 days; dehydrate in $95 \%$ alcohol, and transfer to thin celloidin,-and subsequently thick celloidin-made up with $\mathbf{9 5 \%}$ alcohol (not absolute). The sections may be stained in safranin, quickly dehydrated and cleared with carbol-xylene and mounted in balsam, either without a cover-glass, or in thick balsam melted by heat and applied warm. Unless such precautions are taken, the solvent of the balsam may in time dissolve out the granules of blackened fat.

Paraffin does not afford as good a preservation of the more labile fat globules. Dehydrate in equal parts of $95 \%$ and absolute alcohol, clear before the infiltration in carbol-xylene or chloroform. Paraffin in the sections should be dissolved out by carbol-xylene in preference to xylene ( $\$ 143)$. 
§ 228. Dichromate mordantage, with subsequent copper or iron hematoxylin stain, appears to rest upon the power of the fat or lipoid to reduce the dichromate and thus take on a mordantage which gives the basis for the subsequent staining. So far, it has not been possible by this technique to preserve, in paraffin or celloidin sections, the individual fat granules, but it is nevertheless a useful method for the differentiation of lipoid-containing cells.

Fix tissue 2 days in Zenker's with but $1 / 10$ or $1 / 5 \%$ of acetic acid or Helly's fluid, mordant 4 days or longer in Müller's fluid at 35 to $38^{\circ} \mathrm{C}$., wash in water, imbed in paraffin using chloroform as the clearer $(\S 54)$. Stain sections with the copper hematoxylin ( $\$ 95)$. Lipoidcontaining cells, myelinic nerve fibers, erythrocytes, etc., a dark blue. It should be also remembered that other structures may also be stained by this technique.

The freezing microtome may be used with such tissue and stain, as has been done by Benda and Fischler [6].

\section{B. Glycogen.}

Glycogen is soluble in aqueous media, and while it may be retained by a short fixation in several fluids, the best preservative of it is $95 \%$ alcohol ( $82 \%$-absolute).

$\S 229$. The Iodine Method. [16]. Fix tissue in $95 \%$ or $82 \%$ alcohol. Imbed in paraffin. Spread the sections using instead of the water, the iodine stain for glycogen, which is made up as follows: iodin, 11/2 grams; potassium iodid, 3 grams; sodium chlorid, 11/2 grams; distilled water, 300 c. c. Spread sections may be stained or restained by immersing in the iodin solution which will color the glycogen a mahogany red. For very soluble glycogen, 50\% alcohol may be employed instead of the water in making up the stain. In mounting, dissolve the paraffin with xylene, drain, place on the preparation melted yellow vaseline, cover, seal with shellac or balsam.

$\S 230$. Best's Method. [5]. While rather complicated, this is generally recognized as the best method for the demonstration of small quantities of glycogen, especially when it is desired to see the relation of the granules to the protoplasm.

Fix tissue in $95 \%$ alcohol; imbed (preferably) in celloidin ( $\$ 67)$; if paraffin is used, after dehydration $(\$ 51)$ place the pieces of tissue in pure acetone for 15 minutes, xylene 20 minutes, xylene paraffin 1 hour, pure paraffin ( $\S 52) 1$ hour. Stain sections in Delafield's hematoxylin ( $\$ 91)$ strongly, rinse and differentiate (if necessary) and stain in the following special carmine stain which had been 
previously prepared. (a). Carmine, 1 gram; ammonium chlorid, 2 grams; lithium carbonate, 0.5 grams; distilled water, 50 c. c. Bring the mixture to a boil. When it is cool, add 20 c. c. $10 \%$ am- . monia. Preserve the solution in the dark; after 2 to 3 days it is ready for use and retains its staining quality for a few weeks only. (b) When ready to stain, filter the above solution (a), and add to 2 parts of the stain, 3 parts of $10 \%$ ammonia solution, and 6 parts of methyl alcohol.

Stain sections 1 hour, differentiate in a mixture of 2 parts of methyl alcohol, 4, parts of absolute alcohol, 5 parts of water; rinse with $82 \%$ alcohol, dehydrate, clear and mount in balsam. Glycogen stained an intense red.

In working with glycogen it is sometimes necessary to apply as a control the digestion of the glycogen in one or more sections or a part thereof, by means of saliva.

C. Amyloid.

Amyloid, a form of connective tissue degeneration, resembles glycogen in some of its physical (not chemical) properties and staining reactions. Two methods for its demonstration may be mentioned:

$\S 231$. Iodine Method. Practically any fixer may be used (Zenker's fluid). Paraffin, celloidin, or (better) frozen sections may be used. Stain sections with the iodine solution (\$229) for several minutes; rinse in distilled water and transfer to glycerin or glycerin-jelly $(\S 151,155)$ in which they may be mounted. Seal the preparations ( $\$ 162-)$. The amyloid a reddish-brown; the stain, however, will fade in the course of a few months.

$\S 232$. Gentian Violet, among other anilin stains, colors amyloid differentially (metachromasia). Stain paraffin sections for several minutes in a $1 \%$ solution of the stain; rinse and differentiate in $1 \%$ acetic acid; wash thoroughly with distilled water, and (a) mount in glycerin-jelly, or (b) dry in the air, treat with xylene and mount in balsam. In the latter procedure, the staining may be applied before removing the paraffin.

\section{Mucus.}

Mucous substances (mucins, mucinoids) possess acid properties combining with alkalis and bases (heavy metals), such combinations swelling up or dissolving in water. Acetic acid, alcohol and picric acid also precipitate these substances. For the fixation of mucus, 
most fixers may be used,-Mercuric chlorid, Zenker's fluid, Flemming's fluid or Hermann's fluid. If, however, it is desired to preserve the mucus granules (mucinogen?) in the cell-body, alcoholic or picric acid fixers are usually required, and the tissue at no time subsequently should be placed in water or aqueous solutions. Because of its acid character mucus stains with basic stains, the first two methods given below being very selective. If cell granules are desired, remember the caution as to the avoidance of water.

$\S 233$. Mucicarmine. ( $\S 103)$. Fix as recommended above. Stain paraffin sections 1 to 24 hours; wash in water, dehydrate, clear and mount in balsam. It may be used diluted with $50 \%$ or $67 \%$ alcohol.

$\S$ 234. Muchematein. ( $\$ 93)$. Employ in the same manner as mucicarmine. The alcoholic formula is indicated if cell granules are to be preserved.

Staining of the nuclei with hematoxylin $(\$ 88,89)$ or carmine $(\S 98)$ or elastin stain ( $\$ 125)$ may be previously given; a picro-fuchsin stain may follow it. Excess of alum (aluminium salts) or presence of acid must be avoided, hence wash thoroughly with water if there has been previous staining.

$\S 235$. Basic anilin stains usually give sharp, often differential stains (metachromasia) for mucus. Methylene blue, toluidin blue, or gentian violet may be recommended. Safraniı after Flemming's fluid fixation often gives a delicate stain for mucus.

\section{E. Micro-chemical Tests.}

Micro-chemical tests for inorganic elements must occasionally be employed, particularly in cytological work. The methods have been largely elaborated by Macallum to whose papers references are given.

$\S 236$. Iron. (a). Berlin Blue Reaction. Alcohol fixation and the freezing microtome should be employed. Rinse sections in distilled water, $2 \%$ potassium ferrocyanate, 4 to 5 hours, $1 \% \mathrm{Hcl}$ alcohol several hours, rinse in water, dehydrate, clear with clove oil; balsam. A carmine stain may be given,-before or afterwards.

(b) Ammonium Sulphide Reaction. Place sections in ammonium sulphide solution (freshly prepared) for 5 to 20 minutes; (dark green color); rinse quickly with water, dehydrate, clear in clove oil, mount in balsam. 
Free iron may also be demonstrated by the use of a pure aqueous solution of hematoxylin (dark-blue color); other metals, however, also unite with hematoxylin (e. g., calcium).

$\S 237$. Masked Iron does not give the reaction with pure aqueous hematoxylin [31, 34]. Macallum applies the ammonium sulphide at higher temperature $\left(60^{\circ}\right.$ C. $)$ and for several days.

$\S 238$. Phosphorus. See Macallums methods [31].

§ 239. Potassium. See Macallum's method [33].

$\S$ 240. Chlorides. See Macallum's method [32]. Cautions!

$\S 241$. Calcium stains strongly with aqueous hematoxylin and may often be so demonstrated. To identify the calcium and distinguish from iron, usual chemical tests may be applied [31, 28]. 


\section{REFERENCES.}

1. Altmann, R. Die Elementar Organismen. 2te. Aufl. Veit \& Co., Leipzig, 1894.

2. Bell, E. T. The Staining of Fats in Epithelium and Muscle Fibers. Anat. Record. Vol. IV. No. 5, 1910.

2. Benda, C. Die Mitochondria. Ergeb. d. Anat. u. Entwicklungsges. Vol. XII, pp. 743-781. 1901.

4. Berg, W. Die Fehlergrösse bei den histologischen Methoden. Berlin, 1908. Also: Arch. f. mikr. Anat., Vol. LXII, 1903; Vol. LXV, 1905.

5. Best, F. Ueber Karminfärbung des Glykogens und der Kerne. Zeitschr. f. wiss. Mikr. Vol. XXIII, 1906.

6. Ehrlich, P., Krause, R., Mosso, M., Rosin, H., \& Weigert, P.; Editors. Enzyklopaedie der Mikroskopischen Technik. Urban \& Schwarzenberg. Berlin, 1910.

7. Fischer, A. Fixierung, Färbung und Bau des Protoplasms. Kritische Untersuchungen über Technik $u$. Theorie in den neuen Zellforschung. Jena, 1899.

8. Gage, S. H. \& Susanna P. Staining and permanent Preservation of histological Elements isolated by means of Caustic Potash and Nitric Acid Proc. Amer. Soc. Micr. Vol. XI, 1889. pp. 34-45.

9. Gage, S. H. Notes on albuminizing slides for the more certain fixation of serial celloidin sections. Proc. Amer. Soc. Micr. Vol. XIII, 1891, pp. $79-84$.

10. Gage, S. H. Methods of Decalcification in which the Structural Elements are preserved. Proc. Amer. Soc. Micr. Vol. XIV. 1895. pp. 121-124.

11. Gage, S. H. An aqueous Solution of Hematoxylin that does not readily deteriorate. Proc. Amer. Soc. Micr. Vol. XIV. 1892. pp. 125-127.

12. Gage, S. H. Agnes Claypole's method of fixing paraffin sections to the slide. The Observer, Vol. VI. 1895. pp. 60-61. See also: Agnes Claypole; Amer. Micr. Soc. Trans. 1894. pp. 66 \& 127.

13. Gage, S. H. On the Use of Formalin as a dissociating Medium. The Micr. Bull. \& Sci. News. Vol. XII, 1895. pp. 4-5.

14. Gage, S. H. Improvements in oil-sectioning with celloidin. Proc. Amer. Soc. Micr. Vol. XVII, 1895 . pp. 361-370.

15. Gage, S. H. Notes on the Isolation of the tissue Elements. Proc. Micr. Soc. Vol. XIX, 1897. pp. 179-181.

16. Gage, S. H. Permanent Preparations of Tissues and Organs to show Glycogen. Iodin stain; Mounting in yellow Vaseline and Xylene balsam. Proc. Amer. Micr. Soc. 1906. Vol. XXVIII, pp. 203-205. See also: Amer. Journ. Anat. Vol. IV, No. 2, 1904. 
17. Gage, S. H. The Microscope. Comstock Publ. Co., Ithaca, N. Y. 10th Ed. 1908.

18. v. Gehuchten, A. Anatomie du Système Nerveux de 1'Homme. 1906. 4 me. Ed. p. 340.

19. v. Guyer, M. F. Animal Micrology. Univ. of Chicago Press. Chicago, 1906.

20. Hall, W. J. \& Herxheimer, G. Methods of Morbid Histology and Chemical Pathology. Edinburgh \& London, 1905.

21. Hardesty, Irving. Neurological Technique. Univ. of Chicago- Press. Chicago, 1902.

21a. Hardesty, Irving. Laboratory Directions for Histology. Blakiston. 1908.

22. Hastings, T. W. A modified Nochts Stain. The Johns Hopkins Hospital Bull. Vol. XV. 1904. pp. 122-123. See also: Journ. Exper. Med. Vol. III, 1905. p. 265.

23. Heidenhain, M. Ueber Kern und Protoplasma. Leipzig, W. Engelmann. 1892.

24. Heidenhain, M. Ueber die zweckmässige Verwendung des Congo und anderer Amidoazokörper sowie über neue Neutralfarben. Zeitschr. f. wiss. Mikr. Bd. XX. 1903. p. 179.

25. Heidenhain, M. U.eber Vanadiumhaematoxylin, Picro-blauschwarz und Kongo-Korynth. Zeitsch. f. wiss. Mikr. Vol. XXV. 1908. p. 397

26. Heidenhain, M. Plasma und Zelle. Erste Abt.: Allg. Anatomie des lebendigen Masses. Jena. 1907.

27. Jenner, A. A new Preparation for Rapidly Fixing and Staining Blood. Lancet, 1899, I. p. 370.

28. v. Kahlden-Giercke. Technik der histolgischen Untersuchungen. 8. Ed. Fischer, Jena, 1909.

29. Faure-Fremiet, A. Mayer, G. Schaeffer. Sur la microchimie des corps gras; application a l'etude des mitochondries. Arch. d'Anat. microscopique. Vol. XII, Fasc. L, 1910. pp. 19-102.

30. Lee, A. B. The Microtomist's Vademecum. 7 Ed. Blakiston, Phila. 1913.

31. Macallum, A. B. Die Methode und Ergebnisse der Microchemie in die biologische Forschung. Ergeb. d. Physiol. Vol. VII, pp. 552-652. 1908.

32. Macallum, A. B. On the Nature of the Silver Reaction in Animal and Vegetable Tissues. Proc. Roy. Soc. Vol. LXXVI. 1905. p. 217.

33. Macallum, A. B. On the Distribution of Potassium in Animal and Vegetable Cells. Journ. Physiol. Vol. XXXII. p. 95. 1905. 
34. Macallum, A. B. On a new Method of distinguishing between organic and inorganic Iron. Journ. Physiol. Vol. XXII. 1897. p.92. Also: On the Demonstration of the Presence of Iron in Chromatin by Microchemical Methods. Proc. Roy. Soc. Vol. L, p. 277. 1891.

35. Mallory \& Wright. Pathological Technique. 5th. Ed. 1911. W. B. Saunders \& Co., Philadelphia and London.

36. Mann, Gustav. Physiological Histology. Oxford. 1903.

37. Moore, V. A. A Note on the Use of Anise Oil in Histological Methods with special Reference to its Value in Cutting Serial Sections on the Freezing Microtome. Amer. Month. Micr. Journ. Vol. XV. 1894. p. 373.

38. Myers, B. D. The Chiasma of the Toad (Bufo lent.) and of some other Vertebrates. Zeitschr. f. Morphol. \& Anthropol. Vol. III. 1900. pp. 183-207.

29. Pappenheim, M. Grundriss mikroskopischer Färbetechnik. Berlin. 1901.

40. Sheldon, R. E. Paraffin-Weigert methods for the staining of nervous tissue, with some new modifications. Folia Neuro-biologica. Vol. VIII. No. 1, 1914. pp. 1-28.

41. Starke, J. Ueber Fettgranula und eine neue Eigenschaft des Osmium tetraoxydes. Arch. f. Anat. u. Physiol. Physiol. Abt. 1895. p. 70.

42. Streeter, G. L. Ueber die Verwendung der Paraffineinbettung bei Markscheidenfärbung. Arch. f. mikr. Anat. Bd. LXII. 1903. p. 734.

43. Strong, O. S. Notes on the Technique of Weigert's Method for Staining Medullated Nerve Fibers. Journ. Comp. Neurol. Vol. XIII. 1903. p. 291. Also: Ibid. Vol. XVI. 1906.

44. Verhoeff, F. H. An improved differential Elastic Tissue Stain. Journ. Amer. Med. Ass'n. Vol. LVI, No. 18, pp. 1326-1327. 1911.

45. Wright, J. H. A rapid Method for the differential Staining of Blood Films and Malarial Parasites. Journ. Med. Research. Vol. VII. 1902. p. 138.

46. Wright, J. H. Revised Directions for making and using the Wright's bloodstain. Journ. Amer. Med. Ass'n. Vol. LV. pp. 1979. Dec. 3, 1910. 


\section{INDEX}

Acetic acid, picric, formol, 12

Acetic acid, 5, 7

Acid hematoxylin (Delafield's), 39

Acid, formic, I 5, 69

fuchsin, 44

hydrochloric, 36,63

nitric, $15,18,63,66$

Osmic, I 5, I $8,69,73,75,85$

sulphuric, I 8

Acid stains, 35

Acid violet, 45

Action of fixer, 7,8

Adjective staining, 35

Albumin fixative, 49

Alcohol, absolute, 95\%, $82 \%, 67 \%$, $50 \%, \mathrm{II}, \mathrm{I} 4,22,5 \mathrm{O}, 5^{\mathrm{I}}$

Alcohol-acetic, I4

Alcohol-aceto-chloroform, I4

Alcohol-acetic-formol, I4

Alcohols as fixers, I 4, 60, 70, $7 \mathrm{I}$

Alcohol, dehydration in, 22, 27, 5 I , 54

Alcohol, grades, preparation of, I I

Alum cochineal, 4I

Alum differentiator, 36,70

Alkaline methylene blue, 42

Ammonium molybdate, 78

Ammonium picrate, 78

Ammonium sulphid (iron test), 88

Amyloid, stains for, 87

Anilin blue, Mallory's connective tissue stain, 44

Anise-seed oil, 33

Babes' safranit, 42

Balsam, Canada, 54, 55

Balsam, camsal, 55

Damar, 52

Euparal, 55

Basic stains, 35,88

Benda's fluid; see Flemmings fluid

Benda's stain for neuroglia, 79

Bensley's neutral gentian, 46

Berlin blue, 82

Berlin blue reaction (iron), 88

Best's method for glycogen, 86

Bleaching; peroxid, perhydrol; see Hermann's fluid

Bleu de Lyon, stain, 45

Blocks, wooden, for imbedding, 28

Blood films, fixing and staining, 80, 8I

Blood methods for, 79

Blood, preserving in bulk, $8 \mathrm{I}$

Blood vessels, silvering, 83

Bone, 63, 65

Borax carmine, 40

Bouin's fluid, see picro-aceto-formol

Boxes, paper, for imbedding, 24, 29

Cajal, methods for neurofibrillae, 7 I
Calcium, tests for, 89

Camsal balsam, 55

Carbol-xylene, 55

Carmalum, 40

Carminates, carmine, stains, 40, 86

Carmine gelatin mass, 82

Carminic acid, 40

Carnoy's fluids, I4, 70, 79

Castor-xylene, 29, 30

Caustic potash, dissociator, 19, 67

Cedarwood oil, clearer, 23

Cell, methods for, 59

Celloidin, staining, of 37

Celloidin method, 20, 26-,

Celloidin, solutions, $2 \%, 6 \%, 12 \%, 27$, 28

$3 / 4 \%, 34,49$

removal of, 48

Chick blastoderm, fixing; see nitric acid

Chloral hematoxylin, 88

Chlorides, test for, 89

Chloroform, clearer, 23, 69

fixer, I4

hardener, 29

Chromatin, stains for, 59

Chromo-aceto-osmic; see Flemming's fluid

Chromo-nitric; see Perenyi's fluid

Clarifier, clarifying, 29, 30

Class, isolated tissue for, $8 \mathrm{I}$

Cleaning mixture for glass, 58

Cleaning slides, cover-glasses, glassware, 57,58

Clearers, clearing, 22, 23, 55

Cochineal, alum, 4I

Collagen, staining, 6r

Collodion; see celloidin

Congelation mass; see freezing

Congo red, 43,69

Connective tissue, 6 I

Copper dichromate-sublimate -acetic, I2, 6I , 72, 79

Copper hematoxylin, 40, 73, 86

Cover-glasses, $57,5^{8}$

Crystallization of paraffin mass, 25

Cytoplasm, fixation, 60

Cytoplasm, granules, 60, 6I

Damar balsam, mounting medium, 52

Decalcification, 63

Dehydration, 22, 27, 54

Delafield's hematoxylin, 39, 69

Dichromate as fixer, 8, I6, 72, 73, 74, 86

Dichromate-acetic, I2

Differentiation, 36,73 '

Digestion method (reticular tissue), 62

Dishes, for paraffin imbedding, 23 
Dissociation, dissociators, $6,16-$

Double staining, double stains, 37

Dry mounting, 52

Ehrlich's acid hematoxylin, 39

Ehrlich-Biondi-Heidenhain triple stain, 42

Elastic fibers (elastin), stains for, 45,62

Eosin, 43

Eosin-methylene blue, 42

Eosinate of methylene blue, 46

Epithelium, silvering, 83 isolation (dissociation) of, 17,18

Erlicki's fluid, I 5

Erythrosin, 43

Ether-alcohol, 27

Euparal, 55

Fastening sections to slide, 47-

Fat, fixation of, 72,84 freezing microtome, use, 84 stains for, 84

Fixation, 7,8

Fixation by injection, 9

Fixation, rules for, 9

Fixers, action of, 7 formulae for, I I-16 list of, 8

Fixing, 6, 7

Flemming's fluid, $13,74,85$

Flemming's triple stain, 43

Formaldehyde, formalin, I4, I 5

Dissociator, 18,68

Formol-dichromate, 12

Free- (hand) sections, 47,48

Freezing method for sectioning, 19, 33, 48,84

Fresh tissue, examination of, 6

Fuchsin acid, 44

Gage's dissociators, 18

Gage's hematoxylin, $3^{8}$

Gage's method for glycogen, 86

Gelatin masses, 82

Gentian violet, $4 \mathrm{I}, 43,46,87,88$

Gilson's fluid; see mercuro-nitric

Glycerin, 53, 55

Glycerin-jelly, 54, 56

Glycogen, fixation, staining, 86

Gold chlorid, methods, 69

Golgi methods, 74

Gram's solution; see iodine, 45

Granules, cytoplasmic, 60 secretion, 6 I

Ground sections, 64

Gum arabic, 33

Hæmalum, Mayer's, 39

Hair, dissociation of, I8, 19

Hardening of tissues, Io celloidin mass, 29

Hasting's stain, 46, 81

Heidenhain's iron hematoxylin, 39

Held's method (nerve cells), 70
Helly's fluid, 12

Hemateates, hematein, 38

Hematoxylin stains, formulæ, 38, 39, 40 stock solution, 38

Hermann's fluid, I3

Herxheimer's stain, 85

Horn, dissociation of, I 8, 19

Hydrochloric acid decalcifier, 63

Hydrochloric acid carmine, 4I

Imbedding, celloidin, methods, 20, 26 paraffin, 20, 22

Impregnations, 36

Indifferent fluids, 16

Indophenol, 85

Infiltration, (imbedding methods), 22, 26,33

Injection, fine, dense, masses, 82,83

In toto staining, $35,4 \mathrm{I}, 50$

Intra vitam methylene blue, 76

Iodin, I I , 45, 86, 87

Iron hematoxylin, Heidenhain's, 39

Iron, tests for, 88,89

Isolation , 16-, 66, 68, 69

Jenner's stain, 46, 8I

Labeling slides, 56

Lampblack, gelatin mass, 83

Light green, 45

Lipoids; see fats

Lipoids; foot note, 7 demonstration, 84

Lugol's solution; see iodine, 45

Lyon's blue, stain; see Bleu de Lyon, 45

Maceration, 16

Mallory's connective tissue stain, 44

Marchi methods (degenerating nerve fibers), 73

Mayer: see carmalum, haemalum mucicarmine, Muchematein, paracarmine

Mercuric chlorid, I I

Mercuro-nitric, 13

Methylene blue, 42, 7o, 76

Methyl green, 42

Micro-chemical tests, $5^{8}$

Microtome knife, care of, 58

Mitochondria, fixation and staining, 60, 61

Mordantage, 35

Mounting media, methods, $6,52-$ cells, 53,54

Muchematein, 39, 88

Mucicarmine, 4I, 88

Mucus, 87

Müller's fluid, 15 dissociator, 17

Muscle, isolation of, 66

Muscle, methods for, 66-

Myelin, myelinic nerve fibers, 69

Nail, dissociation of, 18,19 
Nerve cells, isolation, staining, 68

Nerve fibers, isolation, degenerated, 69,73

Nervous system, methods, 68

Neurofibrillæ, 7 I

Neuroglia, stain for, 79

Neutral gentian, 46

Neutral stains, $37,35,36$

Nissl's methylene blue, nethod, 76

Nitric acid, decalcifier, dissociator, fixer, $14,16,63$

Nochts-Hastings stain, 46, 8 I

Normal, salt solution; see physiological salt solution

Orange-fuchsin acid, 62

Orange G., 44

Orcein, 45,62

Orth's fluid; see formol-dichromate

Osmic acid, dissociator, fixer, I 5, I 8, 69, 73,75

Osmium tetroxid; see osmic acid

Pal's method, 73

Paracarmine, $4 \mathrm{I}$

Paraffin, grades, method, 20, 2 I-, 23

Paraffin sections, handling of, 48

Perenyi's fluid, I4

Peroxid, as bleacher; see Hermann's fluid

Phosphorus, 89

Physiological salt solutions, I6

Picric acid, stain, 44

Picro-aceto-formol, 12

Picro-fuchsin, 44, 62

Picro-nitric, I4

Platino-aceto-osmic: see Hermann's fluid

Potassium dichromate, fixer, 16

Potassium hydroxid dissociator, 19, 67

Potassium, tests for, 89

Progressive staining, 35

Pyridin; see Cajal's silver methods, 7 I

Pyroxylin; see celloidin

Quadruple stains, 89

Radium, to prevent electric sections, 26

Regressive staining, 35

Resorcin fuchsin, 45, 62

Reticular tissue, 62

Safranin, 42, 85

Scarlet red, 85

Sealing preparations, 55
Secretion granules, 6I

Sectioning, methods of, 6, 19-

Section staining, 35

Serial sectioning in celloidin, 32

Shellac, cells, rings, 54 sealing, 55,56

Silvering blood vessels, 83

Silver nitrate, 83,7 I, 74

Slides, cleaning, 57

Soluble cotton; see celloidin

Staining, forms of, rules of, 35

Staining, isolated cells, sections, etc., I $8,5 \mathrm{I}, 53,66,67$

Stains, $35^{-}, 3^{8}$

Stock solutions, I I

Storing of tissues, Io

Substantive staining, 35

Sudan III, 85

Sulphalizarinate of sodium, 6I, 79

Sulphuric acid dissociator, $\mathrm{I} 8$

Tellesnicky's fluid; see dichromate acetic

Tigroid substance, 70

Tissues, fixing, 6, 7 hardening, storing, Io

Tissue paper, for handling sections, 3 I

Toluene, as clearer, 22, 2 I

Toluidin blue, 42

Tooth, 63,64

Transferring celloidin sections, 3 I

Triple staining, 37

Triple stain; Ehrlich-Biondi, 42

Flemming's, 43

U1tramarine, gelatin mass, 83

van Gehuchten's, 74

Vascular epithelium, silvering, 83

Verhœeff's elastin stain, 45

von Luko's fluid; see alcohol-acetoformol

Weigert methods, for nerve, $7 \mathrm{I}$

Weigert's copper hematoxylin, 40, 7 I

White connective tissue fibers, 6 I

Wooden blocks, for celloidin imbedding, 28,30

Wright's stain, 46, 8I

Xylene, 23, 50

Xylene, as clearer, 22, 23

Xylol; see Xylene, 22 footnote

Zenker's fluid, I I

Zenker-formol; see Helly's fluid, 12 


THIS BOOK IS DUE ON THE LAST DATE STAMPED BELOW

AN INITIAL FINE OF 25 CENTS WILL BE ASSESSED FOR FAILURE TO RETURN THIS BOOK ON THE DATE DUE. THE PENALTY WILL INCREASE TO 50 CENTS ON THE FOURTH DAY AND TO $\$ 1.00$ ON THE SEVENTH DAY OVERDUE.

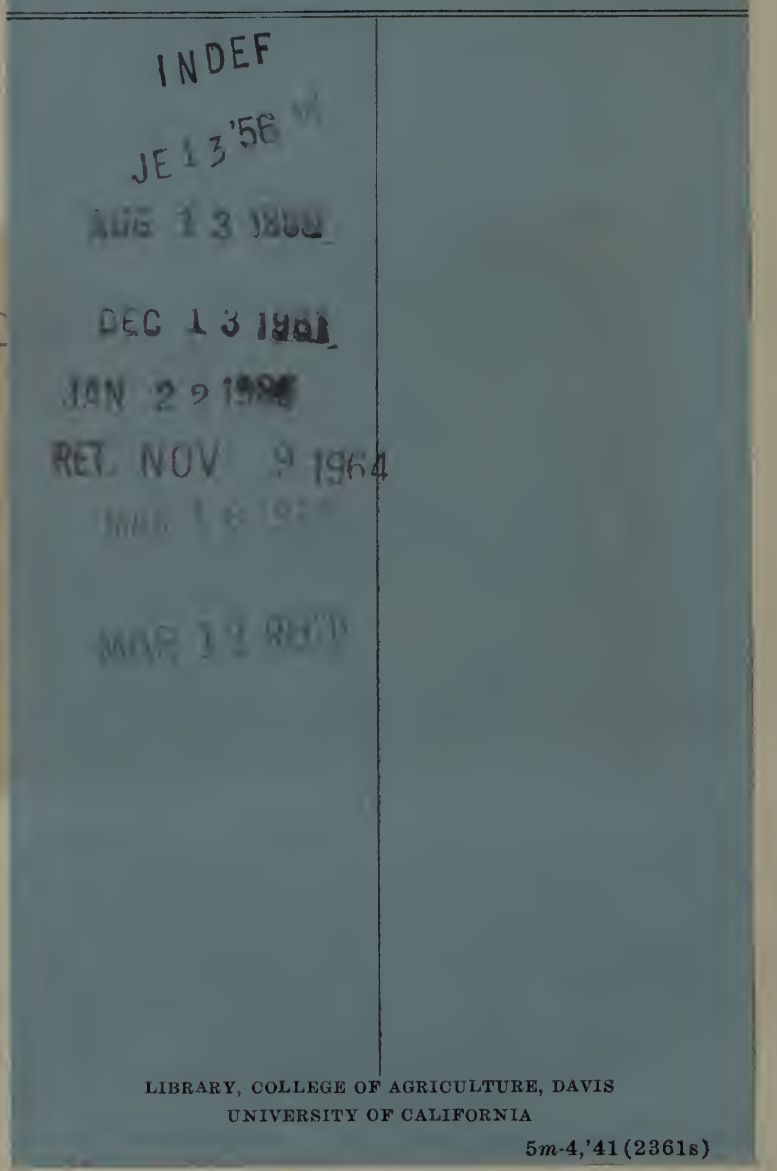




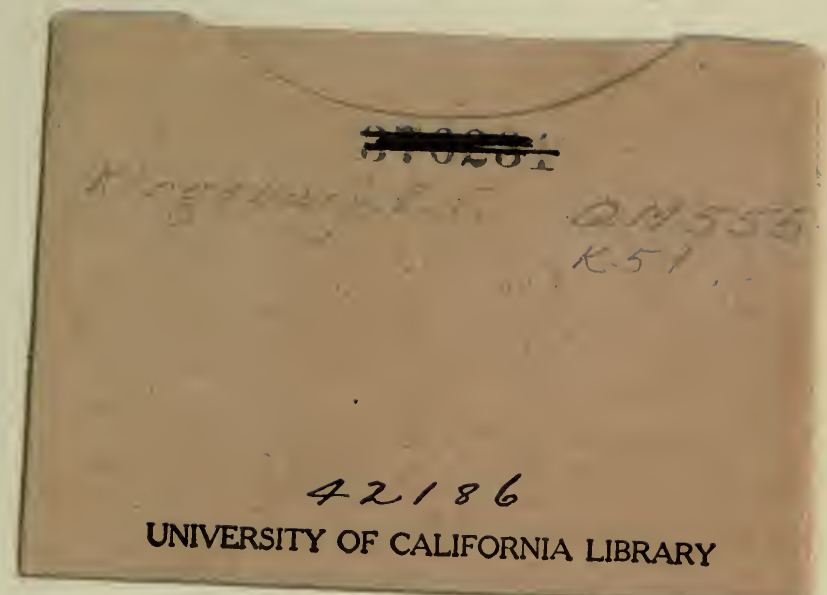


